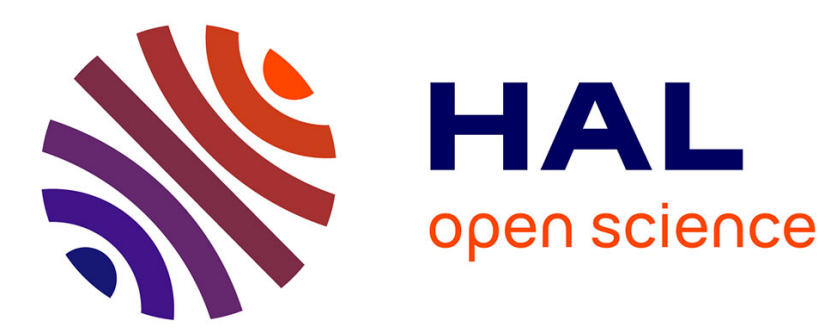

\title{
Majoration du nombre de zéros d'une fonction méromorphe en dehors d'une droite verticale et applications
}

Oswaldo Velásquez Castañón

\section{To cite this version:}

Oswaldo Velásquez Castañón. Majoration du nombre de zéros d'une fonction méromorphe en dehors d'une droite verticale et applications. 2007. hal-00194988

\section{HAL Id: hal-00194988 \\ https://hal.science/hal-00194988}

Preprint submitted on 7 Dec 2007

HAL is a multi-disciplinary open access archive for the deposit and dissemination of scientific research documents, whether they are published or not. The documents may come from teaching and research institutions in France or abroad, or from public or private research centers.
L'archive ouverte pluridisciplinaire HAL, est destinée au dépôt et à la diffusion de documents scientifiques de niveau recherche, publiés ou non, émanant des établissements d'enseignement et de recherche français ou étrangers, des laboratoires publics ou privés. 


\title{
Majoration du nombre de zéros d'une fonction méromorphe en dehors d'une droite verticale et applications
}

\author{
Oswaldo Velásquez Castañón *
}

Décembre 2007

\begin{abstract}
Résumé
On étudie la répartition des zéros des fonctions de la forme $f(s)=h(s) \pm h(2 a-s)$, où $h(s)$ est une fonction méromorphe réelle sur l'axe réel, $a$ un nombre réel. Un de nos résultats établit des conditions suffisantes pour que tous les zéros de $f(s)$, sauf un nombre fini, se trouvent sur la droite $\Re s=a$, appelée droite critique pour la fonction $f(s)$, et qu'ils soient simples, pourvu que tous les zéros de $h(s)$, sauf un nombre fini, soient dans le demi-plan $\Re s<a$. Ce résultat peut être vu comme une généralisation de la condition nécessaire de stabilité de la fonction $h(s)$, dans le théorème d'Hermite-Biehler. On applique ces résultats à l'étude de translatées de la fonction zêta de Riemann et de fonctions $L$, et des intégrales de séries d'Eisenstein, entre autres.
\end{abstract}

Abstract
We study the distribution of the zeros of functions of the form $f(s)=h(s) \pm h(2 a-s)$, where $h(s)$ is a meromorphic function, real on the real line, $a$ a real number. One of our results establishes sufficient conditions under which all but finitely many of the zeros of $f(s)$ lie on the line $\Re s=a$, called the critical line for the function $f(s)$, and be simple, given that all but finitely many of the zeros of $h(s)$ lie on the half-plane $\Re s<a$. This results can be regarded as a generalization of the necessary condition of stability for the function $h(s)$, in the Hermite-Biehler theorem. We apply this results to the study of translations of the Riemann Zeta Function and $L$ functions, and integrals of Eisenstein Series, among others.

\section{Motivation et notations}

Le problème de montrer que les zéros de certaines fonctions sont alignés est lié à l'hypothèse de Riemann, l'un des plus grands problèmes ouverts des mathématiques. On rappelle quelques faits sur ce problème, cf. [34, §§1.1, 2.1, 3.3]. La fonction zêta de Riemann, est la fonction complexe définie par

$$
\zeta(s)=\sum_{n=1}^{\infty} \frac{1}{n^{s}}, \quad \Re s>1
$$

et étendue à tout le plan complexe par prolongement analytique, sauf au point $s=1$, où $\zeta(s)$ a un pôle simple de résidu 1 . Elle satisfait à l'équation fonctionnelle

$$
\zeta^{*}(s)=\zeta^{*}(1-s)
$$

pour $s \in \mathbb{C} \backslash\{0,1\}$, où $\zeta^{*}(s)$ est la fonction zêta complétée, définie $\operatorname{par} \zeta^{*}(s)=\pi^{-s / 2} \Gamma(s / 2) \zeta(s)$, où $\Gamma(s)$ est la fonction gamma. Cette nouvelle fonction a deux pôles simples, en $s=0, s=1$, de résidus 1. On peut réécrire l'équation fonctionnelle sous la forme $\xi(s)=\xi(1-s)$, où $\xi(s)=\frac{1}{2} s(s-1) \zeta^{*}(s)$ est une fonction entière de genre 1.

On voit facilement à partir du produit eulérien de $\zeta(s)$ et de l'équation fonctionnelle que les fonctions $\zeta^{*}(s)$ et $\xi(s)$ ont tous leurs zéros dans la bande $0 \leq \Re s \leq 1$. Pour la fonction zêta $\zeta(s)$ les

${ }^{*}$ Institut de Mathématiques de Bordeaux, UMR 5251, Université Bordeaux 1, 351 Cours de la Libération, 33405 Talence Cedex, France. Courriel: 0swaldo.Velasquez@math.u-bordeaux1.fr 
pôles de $s \Gamma(s / 2)$ sont aussi des zéros; ce sont les entiers négatifs pairs (appelés zéros triviaux de la fonction zêta). La non annulation des fonctions précédentes sur la droite $\Re s=1$ (donc aussi sur $\Re s=0)$ a été démontrée par Hadamard et de la Vallée-Poussin (théorème des nombres premiers). On peut étendre cette région de non-annulation au delà de $\sigma \geq 1$; un des ces résultats est que pour une constante $A>0, \sigma \geq 1-A / \log |\tau|,|\tau|$ assez grand [34, §3.11]

$$
\frac{1}{\zeta(s)}=O(\log |\tau|) \text {. }
$$

On peut trouver un meilleur résultat en [34, §6.19].

Les zéros non triviaux de la fonction zêta de Riemann sont donc dans la bande $0<\Re s<1$, et répartis symétriquement par rapport à la droite $\Re s=1 / 2$, appelée droite critique. L'hypothèse de Riemann est l'assertion que tous ces zéros sont sur la droite $\Re s=1 / 2$. On conjecture de plus que ces zéros sont simples.

Soit $a \in \mathbb{R}, h(s)$ une fonction méromorphe dans le plan complexe, réelle sur l'axe réel. On définit la fonction

$$
f(s)=h(s) \pm h(2 a-s) .
$$

Cette fonction satisfait à l'équation $f(2 a-s)= \pm f(s)$. En particulier, les zéros de $f(s)$ sont symétriques par rapport à la droite $\Re s=a$, appelée droite critique. Un lien entre la répartition des zéros de $h(s)$ et $f(s)$ est établi par le théorème d'Hermite-Biehler (cf. [21, Part III, Lecture 27], [2]) : sous des bonnes conditions de croissance sur $h(s)$, l'alignement et la simplicité des zéros des fonctions $h(s) \pm h(2 a-s)$ sont équivalents à l'absence de zéros de $h(s)$ dans le demi-plan $\Re s \geq a$. Cette dernière condition, connue par le nom de stabilité de $h(s)$, apparaît dans l'étude du comportement asymptotique des solutions de certaines équations différentielles (cf. [1]). Ceci est le cas où $a=0$; les fonctions liées à l'hypothèse de Riemann correspondent au cas $a=1 / 2$ (l'étude des problèmes correspondants à deux valeurs de $a$ sont équivalents).

Dans ce travail, on étude les conséquences d'une relaxation de la condition de stabilité, par l'introduction de zéros de $h(s)$ dans le demi-plan $\Re s \geq a$. Sous certaines conditions, on obtient une borne effective pour le nombre de zéros de $f(s)$ en dehors de la droite critique, ainsi que des informations sur la simplicité de ces zéros. L'idée consiste à compter les zéros de $f(s)$ dans une bande $0<\Im(s)<T$, à l'aide de la méthode standard (cf. [34, $\S \S 9.3,9.4]$ ), puis comparer l'estimation trouvée avec celle du nombre de zéros de $f(s)$ sur la droite critique. Cette idée est inspirée de l'article de P. R. Taylor [31], élève de Titchmarsh.

Fixons quelques notations. Dorénavant, pour $s \in \mathbb{C}$, on note $\sigma=\Re s$ et $\tau=\Im s$. On suppose qu'il existe $\sigma_{0}>a$ tel que $f(s) \neq 0$ pour $\sigma \geq \sigma_{0}$. Par l'équation fonctionnelle, $f(s) \neq 0$ lorsque $2 a-\sigma \geq \sigma_{0}$, c'est-à-dire, lorsque $\sigma \leq 2 a-\sigma_{0}$. Donc, tous les zéros de $f(s)$ sont dans la bande $|\sigma-a|<\sigma_{0}-a$. Étant donné $T>0$, on note

$$
N(T)=\#\{s \in \mathbb{C} \mid f(s)=0,0<\tau<T\},
$$

le nombre des zéros de $f(s)$ tel que $0<\tau<T$, et

$$
N_{0}(T)=\#\{s \in \mathbb{C} \mid f(s)=0, s=a+i \tau, 0<\tau<T\}
$$

le nombre de ces zéros sur la droite critique $\sigma=a$ (on tient compte de la multiplicité des zéros dans $N(T)$ et $N_{0}(T)$ ). On note $N_{0}^{\prime}(T)$ le nombre des zéros critiques, sans tenir compte de leur multiplicité. Il est évident que $0 \leq N_{0}^{\prime}(T) \leq N_{0}(T) \leq N(T)$.

Si l'on pose

$$
L(T)=\#\{s \in \mathbb{C} \mid f(s)=0, \sigma>a, 0<\tau<T\},
$$

l'application $s \mapsto \overline{2 a-s}$ établit une correspondance entre les zéros de $f(s)$ tels que $\sigma>a, 0<\tau<$ $T$ et ceux tels que $\sigma<a, 0<\tau<T$, donc

$$
N(T)-N_{0}(T)=\#\{s \in \mathbb{C} \mid f(s)=0, \sigma \neq a, 0<\tau<T\}=2 L(T) .
$$


Cela montre que $N(T)-N_{0}(T)$ est un nombre pair positif ou nul. On a de plus

$$
0 \leq N(T)-N_{0}(T) \leq N(T)-N_{0}^{\prime}(T) .
$$

C'est cette dernière quantité celle que l'on cherche à estimer à partir d'informations sur $h(s)$.

Cet article est organisé de la façon suivante. Dans un premier temps, on considère le cas où il y a une quantité finie de zéros de $h(s)$ dans le demi-plan $\sigma \geq a$. Au $\S 2$, on obtient une première minoration pour le nombre de zéros sur la droite critique $\sigma=a$ de la fonction $f(s)$. Au §3, et sous certaines conditions de croissance sur $h(s)$, on obtient le résultat central de l'article, le théorème 4, qui fournit une borne pour le nombre de zéros de $f(s)$ en dehors de la droite critique $\sigma=a$, en fonction du nombre de zéros de la fonction $h(s)$ dans le demi-plan $\sigma \geq a$. En conséquence du théorème, on montre au $\S 4$ que la borne inférieure donnée au $\S 2$ donne une bonne estimation pour le nombre de zéros de $f(s)$, et on obtient la repartition globale des zéros de $f(s)$. De plus, dans certains cas on obtient une amélioration du théorème 4. Le lien entre nos résultats et la théorie de la stabilité des polynômes, notamment le théorème d'Hermite-Biehler, est donnée au \$5. Un exemple d'application des résultats, motivé par l'étude d'une première approximation à la fonction zêta de Riemann, est donnée au $\$ 6$. La répartition des zéros des sommes et des différences de translatées de la fonction zêta complétée commence au \$7. La nécessité de travailler avec une quantité infinie de zéros à droite de la droite critique nous amène à la première extension du théorème 4 . On généralise les résultats du $\S 2$ au $\S 8$, puis on établit l'extension souhaitée au $\S 9$. On étudie des fonctions provenantes de certaines intégrales de séries d'Eisenstein sur la surface modulaire aux \$10. Une nouvelle extension du théorème 4 motivée au \$10, nous permettra d'obtenir des résultats sous des conditions de croissance affaiblies pour $h(s)$, au \$11. On utilise aussi nos résultats pour simplifier des résultats récents sur la répartition des zéros d'approximations des fonctions zêta d’Epstein, au §12. Finalement, on généralise au $\$ 13$ nos résultats au cas où $h(s)$ n'a pas la symétrie réelle, et on applique ces généralisations à l'étude de combinaisons de translatées de séries $L$ de Dirichlet. Une annexe à la fin du travail lie nos résultats à d'autres obtenus sous un point de vue legèrement différent.

Les calculs numériques et les graphes des fonctions considérées au $\$ 7$ ont été réalisés à l'aide du système PARI/GP [32].

\section{Zéros sur la droite critique}

Soit $h(s)$ holomorphe et sans zéro sur $\sigma=a$. À partir d'une valeur quelconque de $\arg h(a)$, on définit la fonction

$$
\varphi(\tau)=\arg h(a+i \tau)
$$

par variation continue de l'argument de $h(s)$ le long du segment qui lie $a$ et $a+i \tau$. Une telle fonction est appelée fonction de phase de $h(s)$.

Commençons par un lemme simple, dont on fournit la preuve en vue d'une modification ultérieure.

Lemme 1. Soit $g:[0, \infty) \rightarrow \mathbb{R}$ une fonction continue telle que $g(0) \leq 0$. Posons

$$
\tilde{g}(T)=\#\{x \mid 0 \leq x<T, g(x) \equiv 0 \bmod 1\} .
$$

Alors, pour $T>0$,

$$
\tilde{g}(T) \geq g(T) .
$$

Démonstration. Si $\tilde{g}(T)=\infty$, le résultat est évident. Sinon, soient $x_{1}<\cdots<x_{n}$ les nombres $x_{i} \in\left[0, T\left[\right.\right.$ tels que $g\left(x_{i}\right) \equiv 0 \bmod 1$, par définition $n=\tilde{g}(T)$. Par continuité, $g\left(x_{1}\right) \leq 0, \mid g\left(x_{i}\right)-$ $g\left(x_{i-1}\right) \mid \leq 1$ pour tout $i=2, \ldots, n$ et $\left|g(T)-g\left(x_{n}\right)\right| \leq 1$. On obtient alors

$$
g(T) \leq g(T)-g\left(x_{1}\right)=g(T)-g\left(x_{n}\right)+\sum_{i=2}^{n}\left(g\left(x_{i}\right)-g\left(x_{i-1}\right)\right) \leq n=\tilde{g}(T) .
$$


Cela conclut la preuve.

En fait, on a montré ci-dessus que $\lceil g(T)\rceil \leq \tilde{g}(T)$.

Il faut remarquer que si l'on cherche à en déduire une bonne borne inférieure de $\tilde{g}(T)$, la fonction $g(T)$ doit être presque croissante. Mais ce lemme nous fournit au moins une première estimation pour le nombre de zéros de $f(s)$ sur la droite critique, en utilisant une fonction de phase de $h(s)$.

Lemme 2. Soit $a \in \mathbb{R}$ fixé, $h(s)$ une fonction méromorphe dans le plan complexe, réelle sur l'axe réel, sans zéros ni pôles sur la droite critique $\sigma=a$, avec $h(a)>0$. On considère $\arg h(a+i \tau)$, la détermination continue de l'argument de $h(s)$ sur la droite critique $\sigma=a$ telle que $\arg h(a)=0$. Soit $f(s)=h(s) \pm h(2 a-s)$. Le nombre de zéros de $f(s)$ tels que $0<\tau<T$, sans compter les multiplicités, sur la droite $\sigma=a$ est minoré par

$$
N_{0}^{\prime}(T) \geq \frac{1}{\pi} \arg h(a+i T)-u_{ \pm}
$$

pour $T>0$, où $u_{+}=\frac{1}{2}, u_{-}=1$. La multiplicité de a comme zéro de $f(s)$ est $n_{f, a}=0$ dans le cas ,$+ n_{f, a} \geq 1$ et impaire dans le cas -.

Démonstration. Si $s=a+i \tau$,

$$
\begin{aligned}
f(a+i \tau) & =h(a+i \tau) \pm h(a-i \tau)=h(a+i \tau) \pm \overline{h(a+i \tau)} \\
& =|h(a+i \tau)| e^{i \arg h(a+i \tau)} \pm|h(a+i \tau)| e^{-i \arg h(a+i \tau)} .
\end{aligned}
$$

On considère deux cas :

(i) Lorsque $f(s)=h(s)-h(2 a-s)$,

$$
f(a+i \tau)=2 i|h(a+i \tau)| \sin (\arg h(a+i \tau))
$$

et $h(a+i \tau) \neq 0$, donc $f(a+i \tau)=0$ si et seulement $\operatorname{si} \arg h(a+i \tau) \equiv 0 \bmod \pi$. Donc

$$
N_{0}^{\prime}(T)=\#\left\{\tau \mid 0<\tau<T, \frac{1}{\pi} \arg h(a+i \tau) \equiv 0 \bmod 1\right\} .
$$

Par le lemme 1, et comme $\arg h(a)=0$,

$$
N_{0}^{\prime}(T)=\#\left\{\tau \mid 0 \leq \tau<T, \frac{1}{\pi} \arg h(a+i \tau) \equiv 0 \bmod 1\right\}-1 \geq \frac{1}{\pi} \arg h(a+i T)-1 .
$$

Dans ce cas $f(a)=0$, et comme $f(\sigma)=-f(2 a-\sigma)$, la fonction $f(s)$ change de signe sur la droite réelle au point $s=a$, donc $n_{f, a} \geq 1$ et $n_{f, a}$ est impaire.

(ii) Lorsque $f(s)=h(s)+h(2 a-s)$,

$$
f(a+i \tau)=2|h(a+i \tau)| \cos (\arg h(a+i \tau)),
$$

donc $f(a+i \tau)=0$ si et seulement si $\arg h(a+i \tau) \equiv \frac{\pi}{2} \bmod \pi$. D'après le lemme 1

$$
N_{0}^{\prime}(T)=\#\left\{\tau \mid 0<\tau<T, \frac{1}{\pi} \arg h(a+i \tau) \equiv \frac{1}{2} \bmod 1\right\} \geq \frac{1}{\pi} \arg h(a+i T)-\frac{1}{2} .
$$

On a aussi $f(a)=2 h(a) \neq 0$, donc $n_{f, a}=0$. et

Si la fonction de phase $\varphi(\tau)=\arg h(a+i \tau)$ est croissante, l'estimation dans (2) est optimale,

$$
N_{0}^{\prime}(T)=\left\lceil\frac{1}{\pi} \arg h(a+i T)-u_{ \pm}\right\rceil .
$$

On peut aussi considérer la fonction $f(s)=h^{2}(s)-h^{2}(2 a-s)=f^{-}(s) f^{+}(s)$. Alors

$$
f(a+i \tau)=4 i\left|h^{2}(a+i \tau)\right| \sin (\arg h(a+i \tau)) \cos (\arg h(a+i \tau)),
$$


et les zéros de $f(s)$ sur la droite critique correspondent aux zéros de $f^{+}(s)$ et $f^{-}(s)$ sur cette droite, de façon entrelacée (sans tenir en compte leurs multiplicités) si arg $h(a+i \tau)$ est une fonction croissante.

Par contre, si $\arg h(a+i \tau)$ n'est pas une fonction croissante, le lemme 2 ne nous donne pas de bonne estimation pour $N_{0}^{\prime}(T)$. Il est donc nécessaire de raffiner ce lemme.

Soient $0 \leq x_{1}<x_{2}<\cdots<x_{n}<T$ dans la preuve du lemme 1. On dit que $x_{i-1}$ est un point $\grave{a}$ valeur entière décroissante de $g(x)$, si $g\left(x_{i}\right) \leq g\left(x_{i-1}\right)$. Supposons maintenant que $g\left(x_{i}\right) \leq g\left(x_{i-1}\right)$ pour $k$ valeurs différentes de $i, 1<i \leq n(g(x)$ a $k$ points à valeurs entières décroissantes). Soient

$$
\begin{gathered}
c(T)=\#\left\{i \mid 1<i \leq n, g\left(x_{i}\right)-g\left(x_{i-1}\right)=0\right\}, \\
d(T)=\#\left\{i \mid 1<i \leq n, g\left(x_{i}\right)-g\left(x_{i-1}\right)=-1\right\},
\end{gathered}
$$

alors $k=c(T)+d(T)$ et

$$
\begin{aligned}
g\left(x_{n}\right)-g\left(x_{1}\right) & =(n-1-c(T)-d(T)) \cdot 1+c(T) \cdot 0+d(T) \cdot(-1) \\
& =\tilde{g}(T)-c(T)-2 d(T)-1 .
\end{aligned}
$$

Posons $K(T)=c(T)+2 d(T)-g\left(x_{1}\right)$. On a $\tilde{g}(T)-g\left(x_{n}\right)=K(T)+1$. En outre, on définit $\delta(T)=g\left(x_{n}\right)-g(T)+1$, de façon que

$$
\tilde{g}(T)=g(T)+K(T)+\delta(T) .
$$

On a par définition $0 \leq \delta(T) \leq 2$. La fonction $\delta(T)$ est continue, sauf aux points où elle (ou bien $g(T)$ ) prend des valeurs entières, points où elle est continue à gauche.

Notons certaines propriétés de $K(T)$ :

1) $K(T) \geq 0, K(T) \in \mathbb{Z}$

2) $K(T)$ est une fonction croissante de $T$;

3) si $g(x)$ a $k$ points entiers à valeurs entières décroissantes dans $[0, T[$, alors $K(T) \geq k$. En conséquence $\tilde{g}(T) \geq g(T)+k$.

On applique ce dernier résultat aux fonctions du lemme 2, $g_{1}(\tau)=\frac{1}{\pi} \arg h(a+i \tau)$ et $g_{2}(\tau)=$ $\frac{1}{\pi} \arg h(a+i \tau)-\frac{1}{2}$, pour obtenir l'estimation

$$
N_{0}^{\prime}(T) \geq \frac{1}{\pi} \arg h(a+i T)-u_{ \pm}+k
$$

au lieu de (2).

Lemme 3. Soit $g:[0, \infty[\rightarrow \mathbb{R}$ une fonction continue qui n'a qu'un nombre fini de points à valeurs entières dans tout intervalle $[0, T[, T>0$. Si $g(x)$ n'a qu'un nombre fini de points à valeurs entières décroissantes, alors il existe $k \in \mathbb{Z}$ tel que pour $T>0$ assez grand,

$$
\tilde{g}(T)=\lceil g(T)\rceil+k .
$$

En particulier, $\tilde{g}(T)=g(T)+O(1)$. En particulier, si $g(T)$ est une fonction strictement croissante et $g(0) \in]-1,0]$, alors $k=0$ et

$$
\tilde{g}(T)=\lceil g(T)\rceil .
$$

Démonstration. S'il n'y a qu'un nombre fini de points à valeurs entières décroissantes de $g(T)$, alors la fonction $K(T)$ est ultimement constante, $\delta(T) \neq 1, \delta(T) \neq 2$ pour $T$ assez grand. S'il y a une infinité de points à valeurs entières, on aura ultimement $\delta(T)<1$, ce qui nous donnera $0 \leq \delta(T)<1$. En effet, si $\delta(T) \geq 1$ et s'il existe $T_{1}>T$ tel que $\delta\left(T_{1}\right) \in \mathbb{Z}$, alors pour la première valeur de $T_{1}$ telle que cela arrive, on a $\delta\left(T_{1}\right)=1$ ou 2 (par continuité à gauche de $\delta(T)$ ), absurde. S'il n'y a qu'une quantité finie de points à valeurs entières, on aura soit $0<\delta(T)<1$ pour $T$ assez grand, soit $1<\delta(T)<2$ pour $T$ assez grand. Tout cela nous permet d'écrire

$$
\tilde{g}(T)+\lceil-\delta(T)\rceil=\lceil g(T)\rceil+K(T),
$$


où $K(T)$ est constante, $\lceil-\delta(T)\rceil$ est la constante 0 ou -1 , pour $T$ assez grand.

Si la fonction $\varphi(\tau)=\arg h(a+i \tau)$ satisfait à la condition du lemme 3, on peut obtenir une meilleure estimation pour $N_{0}^{\prime}(T)$ que celle donnée par le lemme 2 dans le cas où cette fonction n'est pas croissante. Une condition sufissante pour cela est que $\varphi(\tau)$ soit ultimement croissante.

\section{Résultat principal}

L'idée de base de la méthode de P. R. Taylor, ayant pour objectif de démontrer la proposition 12, est la suivante : d'abord, on calcule $N(T)$ à l'aide du principe de l'argument. Ensuite, on estime $N_{0}^{\prime}(T)$ avec le lemme 2. La différence $N(T)-N_{0}^{\prime}(T)$ contient un terme d'erreur; on montre que la moyenne de la contribution de ce terme est négligeable grâce à un théorème de Littlewood. Il est à noter que la même idée est à l'origine de la méthode de vérification numérique de l'hypothèse de Riemann imaginée par Turing [8, §8.2].

Théorème 4. Soit $a \in \mathbb{R}, h(s)$ une fonction méromorphe sur $\mathbb{C}$, réelle sur la droite réelle, n'ayant qu'un nombre fini de pôles dans $\mathbb{C}$, un nombre fini de zéros dans le demi-plan $\sigma>$ a, holomorphe et sans zéro sur la droite critique $\sigma=a$. On définit la fonction

$$
f(s)=f^{ \pm}(s)=h(s) \pm h(2 a-s)
$$

(en particulier $f(2 a-s)= \pm f(s)$ ). On suppose que la fonction

$$
F(s)=\frac{h(2 a-s)}{h(s)}
$$

satisfait

(i) pour chaque $\eta>0$, il existe $\sigma_{0}=\sigma_{0}(\eta)>$ a tel que $|F(s)|<\eta$ si $\sigma \geq \sigma_{0}, \tau \in \mathbb{R}$;

(ii) pour chaques $\varepsilon>0$ et $\sigma_{0}>a$, il existe une suite $\left(T_{n}\right)_{n}$ telle que $\lim _{n \rightarrow \infty} T_{n}=+\infty$ et $|F(s)|<$ $e^{\varepsilon|s|}$ pour $a \leq \sigma \leq \sigma_{0},|\tau|=T_{n}, n \geq 1$.

On note aussi, pour une fonction $m(s), n_{m, \sigma>a}$ le nombre de ses zéros réels $\sigma$ tels que $\sigma>a$, $N_{m, \sigma>a}$ le nombre des zéros (réels ou complexes) tels que $\sigma>a, n_{m, a}$ la multiplicité de $s=a$ comme zéro de $m(s)$ (si c'est le cas, sinon $n_{m, a}=0$ ), et $P_{m, \sigma>a}$ le nombre des pôles de $m(s)$, tels que $\sigma>a$ (on considère les multiplicités dans tous les cas). Alors

$$
N(T)-N_{0}(T) \leq N(T)-N_{0}^{\prime}(T) \leq u_{ \pm}-n_{f, \sigma>a}-\frac{n_{f, a}}{2}+P_{f, \sigma>a}+N_{h, \sigma>a}-P_{h, \sigma>a} .
$$

pour $T>0$, où $u_{+}=\frac{1}{2}, u_{-}=1, n_{f, a}=0($ cas +$)$ ou $n_{f, a} \geq 1$ est impaire (cas -$)$. En particulier, tous les zéros de $f(s)$, sauf un nombre fini, se trouvent sur la droite $\sigma=a$ et sont simples. Le membre de gauche de (5) est de plus un nombre positif pair.

Démonstration. Comme $h(a) \neq 0$, on peut supposer $h(a)>0$, puisque si $h(a)<0$, on change $h(s)$ en $-h(s)$ dans l'analyse. On pose $g(s)=1 \pm F(s)$ (selon le signe qui définit $f(s)$ ), de telle façon que $f(s)=g(s) h(s)$. Il faut remarquer que la condition (iii) reste valable, inchangée, pour $g(s)$.

Soit $0<\eta \leq \frac{1}{2}$. Par (i), il existe $\sigma_{0}>\max \{a, 0\}$ tel que $|1-g(s)| \leq \eta$ pour $\sigma \geq \sigma_{0}$; puis $|g(s)| \geq \frac{1}{2}$ et $f(s)$ n'a pas de pôles, $h(s)$ n'a ni de zéros ni de pôles, pour $\sigma \geq \sigma_{0}$ (puisqu'ils sont en nombre fini). Pour $T>0$, on considère le rectangle $R$ défini par

$$
-T \leq \tau \leq T, \quad 2 a-\sigma_{0} \leq \sigma \leq \sigma_{0}
$$

Suivant [34, §9.9], on définit le logarithme de $g(s)$ de la façon suivante : si $\tau$ n'est pas l'ordonnée d'un zéro, en partant d'une valeur pour $\log g\left(\sigma_{0}\right)$ (dans notre cas $\log g\left(\sigma_{0}\right)=0$, puisque $g\left(\sigma_{0}\right)>0$ ), 
on définit $\log g(s)$ par variation continue le long des segments qui lient $\sigma_{0}, \sigma_{0}+i \tau$ et $s$ dans l'ordre. Si $\tau$ est l'ordonnée d'un zéro, on définit

$$
\log g(s)=\lim _{\delta \rightarrow 0^{+}} \log g(s+i \delta) .
$$

On peut remarquer que sous les conditions données, $\log g(s)$ ne dépend pas de $\sigma_{0}$, pour $\sigma_{0}$ assez grand.

L'application $\sigma \mapsto 2 a-\sigma$ établit une correspondance entre les zéros et pôles $s=\sigma$ de $f(\sigma)$ réels, avec $\sigma>a$, et les zéros et pôles avec $\sigma<a$. Donc l'excès du nombre de zéros réels de $f(s)$ $\left(2 n_{f, \sigma>a}+n_{f, a}\right)$ sur le nombre de pôles (réels ou complexes) de $f(s)$ est

$$
2 n_{f, \sigma>a}+n_{f, a}-2 P_{f, \sigma>a} .
$$

Si $T$ n'est pas l'ordonnée d'un zéro, par le principe de l'argument, on a

$$
2 N(T)+2 n_{f, \sigma>a}+n_{f, a}-2 P_{f, \sigma>a}=\frac{1}{2 \pi} \Delta_{R} \arg f(s)+C(T),
$$

où $\Delta_{R}$ note la variation dans le rectangle $R$, et $C(T)=0$ pour $T$ assez grand (lorsque $R$ contient tous les pôles de $f(s)$ ), disons $\left.T>T_{f}>0\right)$. Des symétries $\overline{f(s)}=f(\bar{s})$ et $f(2 a-s)= \pm f(s)$ de $f(s)$, on en déduit

$$
\Delta_{R} \arg f(s)=4 \Delta \arg f(s),
$$

où $\Delta$ désigne la variation de $\sigma_{0}$ à $\sigma_{0}+i T$, puis à $a+i T$. Puis

$$
\begin{aligned}
N(T) & =\frac{1}{\pi} \Delta \arg f(s)-n_{f, \sigma>a}-\frac{n_{f, a}}{2}+P_{f, \sigma>a}+C(T) \\
& =R(T)+S(T)-n_{f, \sigma>a}-\frac{n_{f, a}}{2}+P_{f, \sigma>a}+C(T),
\end{aligned}
$$

où

$$
R(T)=\frac{1}{\pi} \Delta \arg h(s), \quad S(T)=\frac{1}{\pi} \Delta \arg g(s) .
$$

Si $T$ est l'ordonnée d'un zéro, on définit $R(T)=R(T+0), S(T)=S(T+0)$. Par définition

$$
S(T)=\frac{1}{\pi} \Im \log g(a+i T) .
$$

On prend maintenant le rectangle $R^{\prime}$ défini par

$$
0 \leq \tau \leq T, \quad a \leq \sigma \leq \sigma_{0} .
$$

Par un théorème de Littlewood [34, §9.9],

$$
\int_{\partial R^{\prime}} \log g(s) d s=-2 \pi i \int_{a}^{\sigma_{0}} \nu(\sigma) d \sigma
$$

où $\nu\left(\sigma^{\prime}\right)$ désigne l'excès du nombre de zéros par rapport au nombre de pôles de $g(s)$ dans le rectangle $\sigma^{\prime}<\sigma \leq \sigma_{0}, 0<\tau \leq T$. En particulier

$$
\Re\left(\int_{\partial R^{\prime}} \log g(s) d s\right)=0,
$$

d'où

$$
\pi \int_{0}^{T} S(\tau) d \tau=\int_{a}^{\sigma_{0}} \log |g(\sigma+i T)| d \sigma+\int_{0}^{T} \arg g\left(\sigma_{0}+i \tau\right) d \tau-I\left(\sigma_{0}\right),
$$

avec $I\left(\sigma_{0}\right)=\int_{a}^{\sigma_{0}} \log |g(\sigma)| d \sigma$. Soit $\varepsilon>0$. La condition (iii) entraîne $\log \left|g\left(\sigma+i T_{n}\right)\right|<\varepsilon\left(\sigma+T_{n}\right)$ pour $a \leq \sigma \leq \sigma_{0}, n \geq 1$. Puis

$$
\pi \int_{0}^{T_{n}} S(\tau) d \tau \leq \sigma_{0} \varepsilon\left(\sigma_{0}+T_{n}\right)+\eta T_{n}-I\left(\sigma_{0}\right)
$$


Maintenant on estime la variation de l'argument de $h(s)$ dans le rectangle $R^{\prime \prime}$ donné par

$$
-T \leq \tau \leq T, \quad a \leq \sigma \leq \sigma_{0},
$$

où $\sigma_{0}$ et $T$ sont assez grands de telle façon que tous les zéros et pôles de $h(s)$ restent à l'intérieur de $R^{\prime \prime}$. Soit $\Delta_{R}^{\prime} \arg h(s)$ la variation de l'argument de $h(s)$, obtenue par variation continue le long des segments qui joignent $a-i T, \sigma_{0}-i T, \sigma_{0}+i T, a+i T$. Soit aussi arg $h(a+i T)$ la détermination continue de l'argument de $h(s)$ sur la droite critique $\sigma=a$ telle que $\arg h(a)=0$. Alors

$$
\Delta_{R}^{\prime} \arg h(s)+\arg h(a-i T)-\arg h(a+i T)=2 \pi\left(N_{h, \sigma>a}-P_{h, \sigma>a}\right) .
$$

Las symétrie $\overline{h(s)}=h(\bar{s})$ entraîne, comme dans le cas de $f(s)$, que

$$
\Delta_{R}^{\prime} \arg h(s)=2 \Delta \arg h(s)=2 \pi R(T) \quad \text { et } \quad \arg h(a-i T)=-\arg h(a+i T),
$$

d'où

$$
R(T)-\frac{1}{\pi} \arg h(a+i T)=N_{h, \sigma>a}-P_{h, \sigma>a} .
$$

Donc

$$
R(T)-\frac{1}{\pi} \arg h(a+i T)=N_{h, \sigma>a}-P_{h, \sigma>a}+D(T) .
$$

pour $T>0$, où $D(T)=0$ pour $T$ assez grand (lorsque $R$ contient tous les zéros et pôles de $h(s)$ ), $T>T_{h}>0$. Maintenant, (6) et (9) entraînent

$$
N(T)=\frac{1}{\pi} \arg h(a+i T)-n_{f, \sigma>a}-\frac{n_{f, a}}{2}+P_{f, \sigma>a}+N_{h, \sigma>a}-P_{h, \sigma>a}+C(T)+D(T)+S(T),
$$

ce qui avec le lemme 2, nous donne

$$
N(T)-N_{0}^{\prime}(T) \leq B_{a}+C(T)+D(T)+S(T)
$$

pour $T>0$, où

$$
B_{a}=u_{ \pm}-n_{f, \sigma>a}-\frac{n_{f, a}}{2}+P_{f, \sigma>a}+N_{h, \sigma>a}-P_{h, \sigma>a}
$$

la borne attendue.

Il faut éliminer la contribution de $S(T)$ à la dernière inégalité. Pour $T>0$ fixé, on prend $n$ assez grand pour que $T_{n}>\max \left\{T, T_{f}, T_{h}\right\}$. On intègre pour obtenir

$$
\begin{aligned}
\left(T_{n}-T\right)\left(N(T)-N_{0}^{\prime}(T)\right) & \leq \int_{0}^{T_{n}}\left(N(\tau)-N_{0}^{\prime}(\tau)\right) d \tau \\
& \leq B_{a} T_{n}+\int_{0}^{T_{f}} C(\tau) d \tau+\int_{0}^{T_{h}} D(\tau) d \tau+\int_{0}^{T_{n}} S(\tau) d \tau \\
& \leq B_{a} T_{n}+\int_{0}^{T_{f}} C(\tau) d \tau+\int_{0}^{T_{h}} D(\tau) d \tau+\varepsilon \frac{\sigma_{0}^{2}}{\pi}+\varepsilon \frac{\sigma_{0} T_{n}}{\pi}+\frac{\eta}{\pi} T_{n}-\frac{I\left(\sigma_{0}\right)}{\pi}
\end{aligned}
$$

On divise par $T_{n}$ et on fait $n \rightarrow \infty$, d'où

$$
N(T)-N_{0}^{\prime}(T) \leq B_{a}+\varepsilon \frac{\sigma_{0}}{\pi}+\frac{\eta}{\pi} .
$$

Puis on peut faire $\varepsilon \rightarrow 0$ (n'ayant plus $T_{n}$ ) et, finalement, $\eta \rightarrow 0$ (n'ayant plus $\sigma_{0}$ ), pour obtenir (5).

En particulier, si $B_{a}<2$, on a $N(T)=N_{0}(T)$ puisque $N(T)-N_{0}(T)$ est un nombre entier pair.

\section{Remarques.}


a) La condition d'avoir $h(a) \neq 0$ est artificielle, et elle peut être relaxée. Soit $s=a$ un zéro ou un pôle de $h(s)$, on écrit $h(s)=(s-a)^{m} h_{1}(s)$, où $m \in \mathbb{Z}, h_{1}(a) \neq 0$. Alors on peut factoriser $(s-a)^{m}$ et obtenir une nouvelle fonction $f_{1}(s)$ telle que $f(s)=(s-a)^{m} f_{1}(s)$, en gardant le signe si $m$ est pair, et en le changeant si $m$ est impair. Les conditions sur la nouvelle fonction $F_{1}(s)=h_{1}(2 a-s) / h_{1}(s)$ restent les mêmes. On peut aussi s'occuper facilement des zéros et des pôles de $h(s)$ sur la droite critique, le cas où il y en a et ils sont en nombre fini. Si $s_{0}=a+i \tau_{0}\left(\right.$ avec $\tau_{0} \neq 0$ ) est un zéro de $h(s), h\left(a+i \tau_{0}\right)=0$, alors $\overline{h(a+i \tau)}=h\left(a-i \tau_{0}\right)=0$. On peut factoriser $h(s)=\left(s-s_{0}\right)\left(s-\overline{s_{0}}\right) h_{1}(s)=\left((s-a)^{2}+\tau_{0}^{2}\right) h_{1}(s)$, puis

$$
\begin{aligned}
f(s) & =h(s) \pm h(2 a-s)=\left((s-a)^{2}+\tau_{0}^{2}\right) h_{1}(s) \pm\left((a-s)^{2}+\tau_{0}^{2}\right) h_{1}(2 a-s) \\
& =\left(s-s_{0}\right)\left(s-\overline{s_{0}}\right) f_{1}(s) .
\end{aligned}
$$

La fonction $F(s)$ est la même, donc on revient au cas précédent. On peut éliminer de cette façon un nombre fini de zéros de la fonction $h(s)$. De même pour les pôles.

b) La fonction $F(s)$ dans le théorème satisfait $|F(a+i \tau)|=1$, pour tout $\tau \in \mathbb{R}$. La condition (i), est un peu forte, elle n'est pas satisfaite par les polynômes, qui ont besoin d'une démarche additionnelle pour leur étude, que l'on verra au \$5. Mais cela évite aussi les cas triviaux (eg. si $p(s)$ est un polynôme pair, alors $f^{-}(s)=0$ ). Dans le cas des polynômes, on peut encore obtenir (i) par perturbation du problème initial.

c) Soient $a_{1}, \ldots, a_{m}$ les zéros de $h(s)$ dans le demi-plan $\sigma>a$, et $b_{1}, \ldots, b_{n}$ les pôles de $h(s)$ dans le demi-plan $\sigma<a$, en tenant en compte les multiplicités. On définit

$$
R(s)=\frac{\left(s-a_{1}\right) \cdots\left(s-a_{m}\right)\left(2 a-s-b_{1}\right) \cdots\left(2 a-s-b_{n}\right)}{\left(2 a-s-\overline{a_{1}}\right) \cdots\left(2 a-s-\overline{a_{m}}\right)\left(s-\overline{b_{1}}\right) \cdots\left(s-\overline{b_{n}}\right)}
$$

Alors, $|R(a+i \tau)|=1$ pour $\tau \in \mathbb{R}, \lim _{|s| \rightarrow \infty}|R(s)|=1$, et la fonction

$$
F_{1}(s)=R(s) F(s)
$$

est regulière dans le demi-plan $\sigma \geq a$. Cette fonction satisfait aussi les conditions (i), (ii) du théorème 4. En particulier, $\left|F_{1}(a+i \tau)\right|=1$ pour $\tau \in \mathbb{R}$. Soit $\sigma_{0}>a$ tel que $\left|F_{1}(\sigma+i \tau)\right|<1 / 2$ pour $\sigma \geq \sigma_{0}, \tau \in \mathbb{R}$. Dans l'intervalle $a \leq \sigma \leq \sigma_{0}$, la condition (ii) est èquivalente à la condition que, pour tout $\varepsilon>0,\left|F_{1}(s)\right|=O\left(e^{\varepsilon|\tau|}\right)$ pour $a \leq \sigma \leq \sigma_{0},|\tau|=T_{n}, n$ assez grand. Le principe de Phragmén-Lindelöf ([33, §5.65], [28, Theorem 12.8]) s'applique (en remarquant que la condition sur la suite de valeurs $|\tau|=T_{n}$ est suffisante) pour nous donner $\left|F_{1}(s)\right|<(1 / 2)^{\sigma_{0}-\sigma}<1$ pour $a<\sigma \leq \sigma_{0}$. Donc

(1) si $h(s)$ est une fonction entière sans zéros dans le demi-plan $\sigma \geq a$, alors

$$
|F(s)|<1 \text { pour } \sigma>a
$$

(puisque $F(s)=F_{1}(s)$ dans ce cas);

(2) en général, pour chaque $\eta>0$, il existe $R>0$ tel que

$$
|F(s)|<1+\eta \text { pour } \sigma>a,|s|>R \text {. }
$$

Une condition similaire apparaît dans la généralisation du théorème d'Hermite-Biehler aux fonctions entières de type exponentiel [2, chapitre IV, §5], au lieu des conditions asymptotiques du théorème 4: pour chaque $\varepsilon>0$, il existe une suite de demi-cercles dans le demi-plan $\sigma>a$, centrés en $s=a$ et de rayon convergeant vers l'infini, le long desquels $|F(s)|<e^{\varepsilon|s|}$. Sous cette condition, on peut retrouver (1) et (2), et (1) suffit pour montrer que tous les zéros de la fonction $f(s)$ sont simples et alignés (proposition 39). Certes, c'est une condition plus faible que (i), mais on pourra alors procéder par perturbation du problème initial, comme dans le cas des polynômes au $§ 5$. 
d) On verifiera, pour les fonctions avec lesquelles on va travailler, la condition $F(s)=O\left(|\tau|^{A}\right)$ pour une constante $A>0$, lorsque $|\tau|$ est assez grand, uniformément pour $\sigma \geq a$, au lieu de (iii). Cela est naturel vu qu'on applique ce résultat à l'étude de fonctions provenantes de séries de Dirichlet. Une fonction $F(s)$ avec une telle condition de croissante est appelée d'ordre fini (cf. [33, §9.4]), ou de croissance polynomiale (cf. [14, §5.2]) dans le demi-plan $\sigma \geq a$.

Corollaire 5. Sous les conditions du théorème 4, si h(s) est une fonction entière, alors

$$
N(T)-N_{0}(T) \leq N(T)-N_{0}^{\prime}(T) \leq u_{ \pm}-\frac{n_{f, a}}{2}+N_{h, \sigma>a}-n_{f, \sigma>a} .
$$

En particulier, si $h(s)$ n'a pas de zéro avec $\sigma \geq a$, alors tous les zéros de $f(s)$ sont sur la droite $\sigma=a$ et ils sont simples.

Démonstration. La formule (10) est evidente. Si $n_{h, \sigma>a}=0$, comme le coté droit de cette formule est toujours positif,

$$
0 \leq u_{ \pm}-\frac{n_{f, a}}{2}-n_{f, \sigma>a}
$$

d'où $n_{f, \sigma>a}=0$, et $n_{f, a}=0$ dans le cas,$+ n_{f, a}=1$ dans le cas - . On réintroduit ces valeurs dans la formule (10) pour obtenir $N(T)=N_{0}(T)=N_{0}^{\prime}(T)$.

En fait, le corollaire 5 est équivalent au théorème 4. Un pôle de $h(s)$ est transformé en un zéro en multipliant par un facteur approprié. La raison de garder l'enoncé du théorème pour des fonctions méromorphes est de simplifier l'application du résultat. De même on aurait pu poser $a=0$, une simple translation permettant d'en déduire le cas général.

\section{Répartition globale des zéros}

Sous les conditions du théorème 4, la répartition des zéros de $f(s)$ est essentiellement la répartition des zéros sur la droite critique.

Théorème 6. Sous les conditions du théorème 4, si $\arg h(a+i t)$ est une détermination continue de l'argument de $h(s)$ sur la droite $\sigma=a$, avec $\arg h(a)=0$, alors

$$
N_{0}^{\prime}(T)=\frac{1}{\pi} \arg h(a+i T)+O(1) .
$$

Les zéros des fonctions $f(s)=h(s) \pm h(2 a-s)$ sont ultimement simples et entrelacés. Plus précisement, il existe un entier $d_{f, a} \geq 0$ tel que

$$
N_{0}^{\prime}(T)=\left\lceil\frac{1}{\pi} \arg h(a+i T)-u_{ \pm}\right\rceil+d_{f, a}
$$

pour $T>0$ assez grand. Dans le cas particulier où $\arg h(a+i T)$ est une fonction croissante, on a $d_{f, a}=0$.

Démonstration. Soit $f(s)=h(s)-h(2 a-s)$. La fonction $\varphi(\tau)=\frac{1}{\pi} \arg h(a+i \tau)$ n'a qu'un nombre fini de points à valeurs entières décroissantes. En effet, si ce n'est pas le cas, on aura (4) avec $k$ grand, ce qui dans (5) donne une diminution de $k$ dans la borne à droite. Pour $k$ assez grand, le nombre de droite de (5) serait negatif, ce qui est absurde. On applique le lemme 3 pour obtenir la première partie du résultat. La positivité de $d_{f, a}$ découle de la formule explicite obtenue.

Pour la fonction $f(s)=h(s)+h(2 a-s)$, on choisit $\varphi_{1}(\tau)=\frac{1}{\pi} \arg h(a+i \tau)-\frac{1}{2}$, pour obtenir le même résultat qu'avec $\varphi(\tau)$.

Finalement, on considère $f(s)=h^{2}(s)-h^{2}(2 a-s)=f^{+}(s) f^{-}(s)$. La croissance ultime des points à valeurs entières de $\frac{1}{\pi} \arg h^{2}(a+i \tau)=2 \varphi(\tau)=2 \varphi_{1}(\tau)+1$ correspond à l'entrelacement 
des zéros des fonctions $f(s)=h(s) \pm h(2 a-s)$ sur la droite $\sigma=a$ (voir (3)).

Remarque. Si $h(s)$ est une fonction entière sans zéros avec $\sigma \geq a$, on a $d_{f, a}=0$, par le corollaire 5. En effet, il suffit d'introduire l'estimation (11) dans (10), et voir que cela diminue de $d_{f, a}$ la borne obtenue.

D'un autre côté, la remarque (c,1) au théorème 4 et la proposition 39 entraînent que la fonction $\varphi(\tau)=\arg h(a+i \tau)$ est croissante, d'où on aura également $d_{f, a}=0$. Donc il est naturel de se poser la question suivante, motivée aussi dans la discussion faite à la fin du §2.

Question. Sous les conditions du théorème 4, la fonction $\varphi(\tau)=\arg h(a+i \tau)$ est-elle ultimement croissante? Cela entraînerait le théorème 6 directement.

On établit un lemme qui nous aidera dans la suite.

Lemme 7. Soit $h(s)$ une fonction méromorphe sans zéros sur la droite critique $\sigma=a$. Si $\frac{h^{\prime}(a)}{h(a)}<0$, alors $d_{f, a} \geq 1$ dans la formule (11), pour la fonction $f^{-}(s)=h(s)-h(2 a-s)$.

Démonstration. Soit $\theta(\tau)=\frac{1}{\pi} \arg h(a+i \tau), f(s)=f^{-}(s)$. Un calcul direct montre que

$$
\theta^{\prime}(\tau)=\Re \frac{h^{\prime}(a+i \tau)}{h(a+i \tau)}
$$

et en particulier $\theta^{\prime}(0)=\frac{h^{\prime}(a)}{h(a)}$. Le premier point à valeur entière de $\theta(\tau)$ est $x_{1}=0$ avec $\theta\left(x_{1}\right)=0$. Si $\theta^{\prime}(0)<0$, la fonction $\theta(\tau)$ est décroissante dans un petit intervalle contenant 0 ; par continuité, le deuxième point à valeur entière $x_{2}>0$ satisfait $\theta\left(x_{2}\right) \leq 0$. Donc $x_{1}=0$ est un point à valeur entière décroissante de $\theta(\tau)$. Par l'estimation (4), $d_{f, a} \geq 1$ et

$$
N_{0}^{\prime}(T) \geq \frac{1}{\pi} \arg h(a+i T),
$$

le résultat attendu.

\section{Polynômes et Stabilité. Le théorème d'Hermite-Biehler}

Un polynôme $p(z)=a_{0} z^{n}+a_{1} z^{n-1}+\cdots+a_{n-1} z+a_{n}$ avec $a_{n} \neq 0$ est dit stable si tous ses zéros sont dans le demi-plan $\sigma<0$. On rappelle quelques propriétés des polynômes stables à coefficients réels (cf. [26]) :

(i) Tous les coefficients de $p(z)$ ont le même signe. Si $a_{0}>0$, alors $a_{i}>0$ pour tout $1 \leq i \leq n$ (théorème de Stodola). En particulier, $p^{\prime}(0)=a_{1} \neq 0$.

(ii) La fonction argument phase $\varphi(\tau)=\arg p(i \tau)$ est une fonction croissante de $\tau$. Si on choisit $\arg p(0)=0$, la contribution à l'argument de chaque zéro réel (donc négatif) à l'infini (positif) est $\pi / 2$, et la contribution d'un zéro non réel et son conjugué est $\pi$. Donc

$$
\lim _{\tau \rightarrow+\infty} \arg p(i \tau)=n \frac{\pi}{2} .
$$

Proposition 8. Soit $p(s)$ un polynôme réel stable non constant. Pour chaque $y>0$, on définit la fonction

$$
P(s ; y)=y^{s} p(s) \pm y^{-s} p(-s) .
$$

On note par $N(y ; T)$ le nombre de zéros de $P(y ; s)$ avec $0<\tau<T, N\left(y_{0} ; T\right)$ le nombre de zéros de $P(y ; s)$ avec $\sigma=a$ et $0<\tau<T$, et $N^{\prime}\left(y_{0}, T\right)$ le nombre de ces derniers zéros, sans compter les multiplicités. Alors 
1. Si $y \geq 1$, tous les zéros de la fonction $P(y ; s)$ sont simples et sur la droite $\sigma=0$. De plus

$$
0 \leq N(y ; T)-\frac{T}{\pi} \log y+u_{ \pm} \leq \frac{n}{2} .
$$

2. Si $0<y<1$, alors

$$
N(y ; T)-N_{0}(y ; T) \leq N(y ; T)-N_{0}^{\prime}(y ; T) \leq n,
$$

le degré de $p(s)$.

Démonstration. Il faut remarquer que le théorème 4 ne peut pas être appliqué aux fonctions $f(s)=p(s) \pm p(-s)$, puisque

$$
\lim _{|s| \rightarrow \infty} \frac{p(-s)}{p(s)}= \pm 1
$$

et la condition (i) du théorème n'est pas satisfaite. Pour atteindre ce cas, on introduit la famille de fonctions

$$
P(y ; s)=y^{s} p(s) \pm y^{-s} p(-s)
$$

avec le paramêtre $y>0$. La fonction $F(y ; s)=y^{-2 s} p(-s) / p(s)$ satisfait $F(y ; s)=O\left(y^{-\sigma}\right)$ pour $|s|$ suffisament grand, donc les conditions du théorème 4 pour $y>1$. D'après le corollaire 5, étant $H(y ; s)=y^{s} p(s)$ sans zéros dans le demi-plan $\sigma \geq 0$, tous les zéros de $P(y ; s)$ sont simples et alignés sur la droite $\sigma=0$, pour $y>1$.

Soit $T>0$ qui n'est pas l'ordonnée d'un zéro de $f(s)=P(1 ; s)$. Comme $N(1 ; T)=N(T)$ est fini, par le théorème de Hurwitz [33, §3.45], pour $y>1$ assez proche de 1 (dépendant de $T$ ),

$$
N(T) \leq N(y ; T), \quad N(T)-N_{0}(T) \leq N(y ; T)-N_{0}(y ; T) .
$$

De la deuxième estimation, il est clair que $f(s)=p(s) \pm p(-s)$ aura tous ses zéros sur la droite $\sigma=0$. Par contre, on ne peut rien dire en général à propos de $N_{0}^{\prime}(T)$, donc sur la simplicité des zéros de $f(s)$.

La formule (11) nous dit que pour montrer que $N_{0}^{\prime}(y ; T) \rightarrow N_{0}^{\prime}(T)$ il nous faut voir la continuité de l'argument de $H(y ; i \tau)$ et de la constante $d_{H(y, \cdot), 0}$, en fonction du paramètre $y$. Heureusement, $d_{H(y, \cdot), 0}=0$ puisque l'argument de $H(y ; i \tau)$ est une fonction croissante. En fait

$$
\arg H(y ; i \tau)=\tau \log y+\arg p(i \tau), \quad y \geq 1 .
$$

Donc, si $\frac{1}{\pi} \arg p(i T) \notin \mathbb{Z}$

$$
N(T) \leq \lim _{y \rightarrow 1^{+}} N(y ; T)=\lim _{y \rightarrow 1^{+}} N_{0}^{\prime}(y ; T)=N_{0}^{\prime}(T),
$$

ce qui entraîne la simplicité des zéros de $f(s)$. Cela montre (1).

Si $0<y<1$, on prend $y^{-1}>1$ au lieu de $y$ et $p(-s)$ au lieu de $p(s)$. Par application du corollaire 5, on obtient $N(T)-N_{0}(T) \leq N(T)-N_{0}^{\prime}(T) \leq n$.

Il est étonnant comme on obtient un résultat pour une fonction avec un nombre fini de zéros à partir d'autres fonctions qui en ont une infinité. Mais il est toujours possible d'analyser directement le cas $y=1$, en utilisant un comptage direct des zéros. Le lemme 2 est toujours applicable, ce qui nous donne

$$
N_{0}^{\prime}(T) \geq \frac{1}{\pi} \arg p(i T)-u_{ \pm} .
$$

On a quatre cas à analyser, selon le signe \pm et la parité de $n$; traitons un seul cas, les autres étant analogues. Soit alors $n$ pair et $f(s)=p(s)-p(-s)$. Le zéro $s=0$ est simple, puisque $f^{\prime}(0)=2 p^{\prime}(0) \neq 0$ par (i). Donc $n_{f, a}=1$. En fait, $f^{\prime}(s)$ ne peut changer de signe que pour $s=0$, à cause du fait que ses coefficients son positifs, et il est impossible d'avoir $f^{\prime}(s)$ localmente constante, 
puisque c'est un polynôme. Donc $f(s)$ est strictement croissante ou décroissante sur les intervalles ] $-\infty, 0[$ et $] 0,+\infty[$, ce qui montre que $s=0$ est le seul zéro réel de $f(s)$.

Pour $T>0$ assez grand, on a $N(T)=\frac{n-1}{2}$. En utilisant (13), on obtient

$$
0 \leq N(T)-N_{0}(T) \leq N(T)-N_{0}^{\prime}(T) \leq \frac{n-1}{2}-\arg p(i T)+1<1
$$

pour $T>0$ assez grand. Donc $N(T)=N_{0}(T)=N_{0}^{\prime}(T)$ et tous les zéros de $f(s)$ sont sur la droite $\sigma=0$ et simples.

On reformule le résultat obtenu en termes d'autres fonctions. On écrit $2 q\left(z^{2}\right)=p(z)+p(-z)$, $2 z \cdot r\left(z^{2}\right)=p(z)-p(-z)$. Alors les polynômes $q(z)$ et $r(z)$ ont des racines réelles negatives, simples et entrelacées, la condition nécessaire de stabilité du polynôme $p(z)$ (cf. [12]) ; une condition additionnelle assure l'équivalence entre ces conditions dans le cas des polynômes. Ce résultat d'équivalence est connu comme le théorème d'Hermite-Biehler un théorème qui a été étendu à une classe de fonctions de type exponentiel (cf. [21, Part III, Lecture 27], [2]), incluant les polynômes. Le corollaire 5 (ou le théorème 4) généralise la condition nécessaire de stabilité du théorème d'Hermite-Biehler pour les fonctions entières vérifiant les conditions (i), (ii) du théorème 4. La condition (i) laisse dehors les polynômes et complique leur étude (les polynômes sont facilement analysés avec des méthodes comme celles du annexe A), mais il montre son utilité dans l'étude d'autres fonctions, lorsqu'on admet des zéros à droite de la droite critique, même en quantité infinie (par extension de l'argument).

\section{La fonction $\zeta_{2}(s)$}

Considérons la suite de fonctions méromorphes

$$
\zeta_{n}(s)=\sum_{k=1}^{n} \frac{1}{k^{s}}-\frac{n^{1-s}}{1-s}, \quad n \geq 1 .
$$

Cette suite converge uniformément vers la fonction zêta $\zeta(s)$ sur les compacts du demi-plan $\sigma>0$ privé du point $s=1$, au vue de la formule [34, §3.5]

$$
\zeta(s)=\zeta_{n}(s)+s \int_{n}^{+\infty} \frac{\{x\}-\frac{1}{2}}{x^{s+1}} d x-\frac{1}{2} n^{-s}
$$

valable pour $\sigma>0$, où $\{x\}=x-\lfloor x\rfloor$ est la fonction partie fractionnaire. Le premier terme non trivial de cette suite est celui avec $n=2$; on veut étudier la répartition des zéros de cette fonction.

On pose $h(s)=e^{\alpha s}(s-\beta)$, avec $\alpha, \beta \in \mathbb{R}$, et $a=0$. On étudiera la fonction

$$
f(s)=h(s)-h(-s)=e^{\alpha s}(s-\beta)+e^{-\alpha s}(s+\beta)=2 s \cosh (\alpha s)-2 \beta \sinh (\alpha s),
$$

qui satisfait $f(-s)=-f(s)$. Lorsque $\alpha=\log (2) / 2, \beta=1$, on obtient $f(s)=-2^{s / 2}(1-s) \zeta_{2}(s)$.

L'annulation d'un quelconque des paramètres $\alpha, \beta$ nous amène dans des cas triviaux. Si $\alpha=0$, on a $f(s)=2 s$, et si $\beta=0, f(s)=2 s \cosh (s)$, des fonctions ayant que des zéros réels. Le cas $\alpha<0$ est facilement ramené au cas $\alpha>0$ en prenant $-h(-s)$ au lieu de $h(s)$.

Proposition 9. Soient $\alpha>0, \beta \neq 0$. Tous les zéros non réels de la fonction

$$
f(s)=e^{\alpha s}(s-\beta)+e^{-\alpha s}(s+\beta)
$$

sont simples, et sur la droite $\sigma=0$. Quant aux zéros réels de $f(s)$ :

(i) Si $\beta<0, s=0$ est le seul zéro réel de $f(s)$, et il est simple;

(ii) Si $\beta>0$, 
(a) pour $\alpha<\frac{1}{\beta}, s=0$ est le seul zéro réel de $f(s)=0$, et il est simple;

(b) pour $\alpha=\frac{1}{\beta}, s=0$ est le seul zéro réel de $f(s)=0$, et il est triple;

(c) pour $\alpha>\frac{1}{\beta}, f(s)$ a trois zéros réels simples $s=0, s=\gamma>0$ et $s=-\gamma$.

Démonstration. La fonction

$$
F(s)=\frac{h(-s)}{h(s)}=e^{-2 \alpha s}\left(1+\frac{2 \beta}{s-\beta}\right)
$$

satisfait les conditions du théorème 4. Donc

$$
0 \leq N(T)-N_{0}(T) \leq N(T)-N_{0}^{\prime}(T) \leq 1-n_{f, \sigma>0}-\frac{n_{f, 0}}{2}+N_{h, \sigma>0} \leq \frac{3}{2}
$$

puisque $n_{f, 0} \geq 1$ est impaire, et $N_{h, \sigma>0} \leq 1$. Maintenant $N(T)-N_{0}(T)$ est pair, donc $N(T)=$ $N_{0}(T)$ et $N_{0}(T) \leq N_{0}^{\prime}(T)+1$.

(i) Supposons $\beta<0$. Alors $N_{h, \sigma>0}=0, n_{f, 0} \geq 1$ et

$$
0 \leq N(T)-N_{0}(T) \leq N(T)-N_{0}^{\prime}(T) \leq 1-\frac{n_{f, 0}}{2} \leq \frac{1}{2},
$$

donc $N_{0}^{\prime}(T)=N_{0}(T)=N(T)$, et tous les zéros de $f(s)$ sont simples et sur la droite $\sigma=0$ par le corollaire 5 .

(ii) On suppose maintenant $\beta>0$. Alors $N_{h, \sigma>0}=1$ et

$$
0 \leq N(T)-N_{0}(T) \leq N(T)-N_{0}^{\prime}(T) \leq 2-n_{f, \sigma>0}-\frac{n_{f, 0}}{2},
$$

On calcule $f^{\prime}(0)=2(1-\alpha \beta)$ et $\frac{h^{\prime}(0)}{h(0)}=\alpha-\frac{1}{\beta}<0$.

(a) Si $\alpha<\frac{1}{\beta}$, le lemme 7 nous permet de réduire d'une unité la borne dans (14), puisque $\frac{h^{\prime}(0)}{h(0)}<0$. Donc

$$
N(T)-N_{0}(T) \leq N(T)-N_{0}^{\prime}(T) \leq 1-n_{f, \sigma>0}-\frac{n_{f, 0}}{2} \leq \frac{1}{2},
$$

d'où $N(T)=N_{0}(T)=N_{0}^{\prime}(T)$, puis $n_{f, \sigma_{0}}=0$ et $n_{f, 0}=1$.

(b) Si $\alpha=\frac{1}{\beta}$, alors $f^{\prime}(0)=0$, d'où $n_{f, 0} \geq 3$. Cela dans (14) entraîne $N(T)=N_{0}(T)=N_{0}^{\prime}(T)$ et $n_{f, \sigma_{0}}=0$.

(c) Si $\alpha>\frac{1}{\beta}$, alors $f^{\prime}(0)<0$, donc il existe $x^{\prime}>0$ (et proche de 0 ) tel que $f\left(x^{\prime}\right)<0$. Mais

$$
\lim _{\sigma \rightarrow+\infty} f(\sigma)=+\infty
$$

d'où il existe $\gamma>x^{\prime}>0$ tel que $f(\gamma)=0$, et $n_{f, \sigma>0} \geq 1$. Cela dans (14) nous donne encore $N(T)=N_{0}(T)=N_{0}^{\prime}(T)$ et $n_{f, 0}=1$.

Corollaire 10. Les zéros de la fonction

$$
\zeta_{2}(s)=1+2^{-s}-\frac{2^{1-s}}{1-s}
$$

sont tous simples et sur la droite $\sigma=0$.

Démonstration. Lorsque $\alpha=\log (2) / 2, \beta=1$, on obtient dans la proposition $9 f(s)=$ $-2^{s / 2}(1-s) \zeta_{2}(s)$, avec $0<\alpha<\frac{1}{\beta}$. 


\section{Translatées de la fonction zêta de Riemann}

On considère les différences des translatées de la fonction zêta completée, donnés pour $\alpha>0$ par

$$
f_{\alpha}(s)=\zeta^{*}(s+\alpha)-\zeta^{*}(s-\alpha) .
$$

Ces fonctions s'écrivent sous la forme $f_{\alpha}(s)=h_{\alpha}(s)-h_{\alpha}(1-s)$, où $h_{\alpha}(s)=\zeta^{*}(s+\alpha)$. Dans ce cas $a=\frac{1}{2}$.

Dans un premier temps, on considère $\alpha>\frac{1}{2}$. Sous cette condition, tous les zéros de $h_{\alpha}(s)$ sont à gauche de la droite critique, mais ses pôles vont pousser quelques zéros en dehors de la droite. Ce cas a été étudié par P. R. Taylor en [31, §1], dans le but d'étudier la répartition des zéros de la fonction $f_{1 / 2}(s)=\zeta^{*}\left(s+\frac{1}{2}\right)-\zeta^{*}\left(s-\frac{1}{2}\right)$.

Proposition 11. Soit $\alpha>\frac{1}{2}, \alpha^{*} \approx 6.81707$ tel que $\left(\zeta^{*}\right)^{\prime}\left(\frac{1}{2}+\alpha^{*}\right)=0$. Tous les zéros non réels de la fonction

$$
f_{\alpha}(s)=\zeta^{*}(s+\alpha)-\zeta^{*}(s-\alpha)
$$

sont sur la droite $\sigma=\frac{1}{2}$. Quant aux zéros réels :

1. $s=\frac{1}{2}$ est un zéro simple de $f_{\alpha}(s)$ si $\alpha \neq \alpha^{*}$, triple si $\alpha=\alpha^{*}$;

2. $f_{\alpha}(s)$ a deux zéros réels simples $s=\rho_{\alpha}$ et $s=1-\rho_{\alpha}$, où $\left.\rho_{\alpha} \in\right] 1+\alpha,+\infty[$. Ces sont les seuls zéros réels de $f_{\alpha}(s)$ différents de $s=\frac{1}{2}$.

Démonstration. Suivant le théorème 4 , on pose

$$
F_{\alpha}(s)=\frac{\zeta^{*}(s-\alpha)}{\zeta^{*}(s+\alpha)}
$$

Par la formule complexe de Stirling [33, §4.42], pour $\sigma \geq \frac{1}{2},|s|$ suffisament grand

$$
\log \Gamma\left(\frac{1}{2} s+\frac{1}{2} \alpha\right)=\left(\frac{s}{2}+\frac{\alpha}{2}-\frac{1}{2}\right) \log \left(\frac{s}{2}\right)-\frac{s}{2}+\frac{1}{2} \log 2 \pi+O\left(\frac{1}{|s|}\right),
$$

et $\zeta(s)=1+O\left(2^{-\sigma}\right)$ lorsque $\sigma \geq \sigma_{0}$ puis

$$
\begin{aligned}
\log h_{\alpha}(s) & =\left(-\frac{1}{2} s-\frac{1}{2} \alpha\right) \log \pi+\log \Gamma\left(\frac{1}{2} s+\frac{1}{2} \alpha\right)+\zeta(s+\alpha) \\
& =s\left(\frac{1}{2} \log (s)+O(1)\right),
\end{aligned}
$$

d'où $\lim _{\sigma \rightarrow \infty} h_{\alpha}(\sigma)=+\infty$.

En plus, pour $|s|$ suffisament grand

$$
F_{\alpha}(s)=(2 \pi)^{\alpha} s^{-\alpha}\left(1+O\left(|s|^{-1}\right)\right) \frac{\zeta(s-\alpha)}{\zeta(s+\alpha)} .
$$

Donc, pour $\sigma$ assez grand

$$
F_{\alpha}(s)=O\left(\sigma^{-\alpha}\right)
$$

uniformément en $\tau$, la condition (i) du théorème 4 .

Pour $\tau$ suffisament grand, on a $\zeta(s)=O\left(|\tau|^{A}\right)$ uniformément sur un demi-plan quelconque $\sigma \geq \sigma_{\alpha}$ fixé $[34, \S 5.1]$ (avec $A=A\left(\sigma_{\alpha}\right)$ ), dans notre cas $\sigma_{\alpha}=1 / 2-\alpha$. En plus, de (1), pour $\sigma \geq 1 / 2, \alpha \geq 1 / 2$,

$$
\frac{1}{\zeta(s+\alpha)}=O(\log |\tau|) .
$$

Donc, pour $\sigma \geq 1 / 2$, on a $\sigma-\alpha \geq \sigma_{\alpha}$ et $\sigma+\alpha \geq 1$, d'où

$$
F_{\alpha}(s)=O\left(|\tau|^{A} \log |\tau|\right),
$$

uniformément en $\sigma \geq 1 / 2$, la condition (ii) du théorème 4 . 
La fonction $f_{\alpha}(s)$ est méromorphe, avec des pôles simples aux points $s=-\alpha, 1-\alpha, \alpha, 1+\alpha$. Le point $s=\frac{1}{2}$ est un zéro simple de $f_{\alpha}(s)$, lorsque $\alpha \neq \alpha^{*}$, et pour $\alpha=\alpha^{*}$ un zéro triple. En fait, $f_{\alpha}^{\prime}\left(\frac{1}{2}\right)=2 \zeta^{* \prime}\left(\frac{1}{2}+\alpha\right) \neq 0$ pour $\alpha \neq \alpha^{*}$.

On a vu que $\lim _{\sigma \rightarrow+\infty} h_{\alpha}(\sigma)=+\infty$, et $\lim _{\sigma \rightarrow+\infty} F_{\alpha}(\sigma)=0$, donc $\lim _{\sigma \rightarrow+\infty} f_{\alpha}(\sigma)=+\infty$. En plus

$$
\begin{aligned}
f_{\alpha}(1+\alpha+0) & =\zeta^{*}(1+2 \alpha)-\lim _{h \rightarrow 0^{+}} \zeta^{*}(1+h) \\
& =\zeta^{*}(1+2 \alpha)-\lim _{h \rightarrow 0^{+}} \frac{1}{(1+h)-1}=-\infty .
\end{aligned}
$$

En conséquence, il existe un zéro de $\left.f_{\alpha}(s), \rho_{\alpha} \in\right] 1+\alpha,+\infty\left[\right.$ de multiplicité impaire, et $n_{f, \sigma>\frac{1}{2}} \geq 1$.

Il nous reste à voir que $p_{f, \sigma>1 / 2}=2, p_{h, \sigma>1 / 2}=0$, et $n_{h, \sigma>1 / 2}=0$. Donc du théorème 4

$$
N(T)-N_{0}(T) \leq N(T)-N_{0}^{\prime}(T) \leq \frac{3}{2},
$$

d'où $N(T)=N_{0}(T)$ (et $N_{0}(T) \leq N_{0}^{\prime}(T)+1$ ) pour tout $T>0$. On déduit de cette borne que $\rho_{\alpha}$ est forcément simple et $n_{f, \sigma>\frac{1}{2}}=1$.

En faisant $\alpha \rightarrow \frac{1}{2}$, le théorème de Hurwitz nous donne le résultat suivant, sous la forme originalement posée par P.R. Taylor.

Proposition 12 (P.R. Taylor, $[\mathbf{3 1}, \S 1])$. Tous les zéros non réels de la fonction

$$
f_{1 / 2}(s)=\zeta^{*}\left(s+\frac{1}{2}\right)-\zeta^{*}\left(s-\frac{1}{2}\right)
$$

se trouvent sur la droite $\sigma=\frac{1}{2}$.

L'équation (16) nous dit que, pour $\alpha>1 / 2$, tous les zéros non réels de $f_{\alpha}(s)$ sont simples et sur la droite $\sigma=\frac{1}{2}$, sauf probablement deux (un et son conjugué) zéros doubles. On peut utiliser le lemme 7 pour améliorer ce résultat. On calcule

$$
r(\alpha)=\frac{h_{\alpha}^{\prime}\left(\frac{1}{2}\right)}{h_{\alpha}\left(\frac{1}{2}\right)}=\frac{\left(\zeta^{*}\right)^{\prime}\left(\frac{1}{2}+\alpha\right)}{\zeta^{*}\left(\frac{1}{2}+\alpha\right)}
$$

suivant la valeur de $\alpha>\frac{1}{2}$ (voir la figure 1). Pour $\alpha \rightarrow \frac{1}{2}^{+}$, cette valeur approche $-\infty$. Lorsque

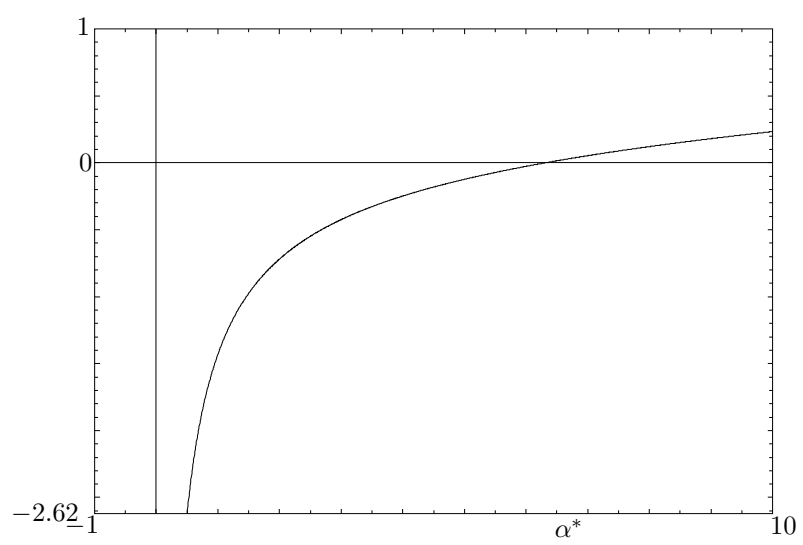

FIG. 1: La fonction $r(\alpha)$

$\alpha<\alpha^{*}$, par le lemme 7, l'écart $d_{f_{\alpha}, a}$ dans le théorème 6 satisfait $d_{f_{\alpha}, a} \geq 1$ et

$$
N_{0}^{\prime}(T) \geq \frac{1}{\pi} \arg h\left(\frac{1}{2}+i T\right)
$$

ce qui entraîne la constante $1 / 2$ au lieu de $3 / 2$ dans (16). Donc tous les zéros non-réels de $f_{\alpha}(s)$ sont simples et alignés sur la droite $\sigma=\frac{1}{2}$, lorsque $\alpha<\alpha^{*}$. La fonction $f_{\alpha^{*}}(s)$ a aussi tous ses 
zéros simples et alignés, sauf $s=\frac{1}{2}$, qui est un zéro triple. Et pour $\alpha>\alpha^{*}$, malheureusement on ne peut pas obtenir mieux que (16).

Bien que le passage de $\alpha>\frac{1}{2}$ à $\frac{1}{2}$ nous fournisse la proposition 12, la manque de stabilité (par rapport au paramètre $\alpha$ ) dans le cas général de $N_{0}^{\prime}(T)$ nous laisse sans information à propos de la simplicité des zéros de $f_{1 / 2}(s)$. On remarque que la méthode originale de $\mathrm{P}$. R. Taylor ne peut pas être appliquée à l'étude de $f_{1 / 2}(s)$; il est pourtant facile de la modifier pour le faire. Dans notre cas, on peut appliquer le théorème 4 à la fonction

$$
\hat{f}(s)=\left(s-\frac{1}{2}\right) f_{1 / 2}(s)=\left(s-\frac{1}{2}\right) \zeta^{*}\left(s+\frac{1}{2}\right)+\left(\frac{1}{2}-s\right) \zeta^{*}\left(\frac{3}{2}+s\right)
$$

et obtenir que presque tous les zéros de $f_{1 / 2}(s)$ sont simples, sauf probablement deux (un et son conjugué, qui pourraient avoir multiplicité double).

Pour la fonction

$$
\hat{f}_{\alpha}(s)=\zeta^{*}(s+\alpha)+\zeta^{*}(s-\alpha)
$$

on a

$$
0 \leq N(T)-N_{0}(T) \leq \frac{5}{2}
$$

(pas de zéro réel connu pour $\hat{f}_{\alpha}(s)$ ), ce qui ne nous permet pas de montrer que tous les zéros de $\hat{f}_{\alpha}(s)$ sont alignés. L'amélioration de l'estimation précédente peut être obtenue en estimant la constante $d_{\hat{f}, a}$. Il faut remarquer que le lemme 7 ne peut pas être appliqué à cette fonction.

D'abord on analyse l'argument de $h_{\alpha}(s)$ sur la droite critique, lorsque $\alpha$ est proche de $\frac{1}{2}$. Pour $\alpha=\frac{3}{5}$, le graphe de la fonction (figure 2)

$$
u(\tau)=\frac{1}{\pi} \arg h_{3 / 5}\left(\frac{1}{2}+i \tau\right)-\frac{1}{2}
$$

nous montre que les trois premiers points $0<x_{1}<x_{2}<x_{3}<21$ à valeurs entières de $u(\tau)$

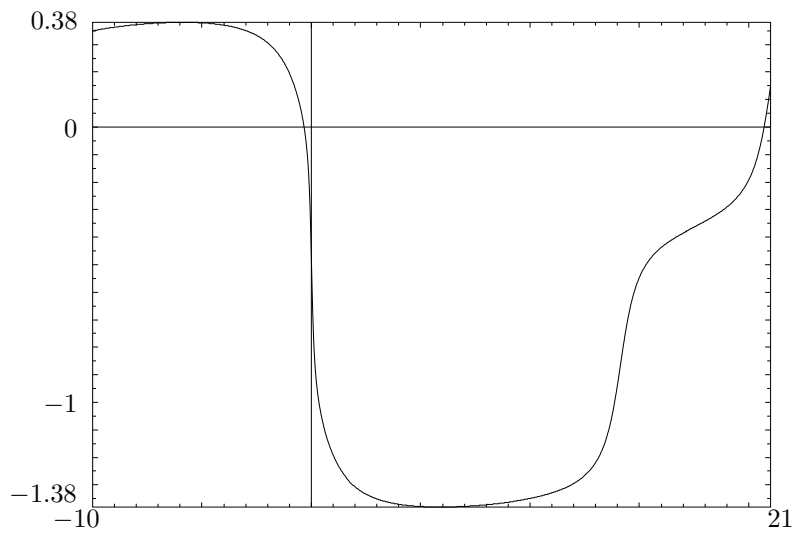

FIG. 2: La fonction $u(\tau)$

satisfont $u\left(x_{1}\right)=-1=u\left(x_{2}\right), u\left(x_{3}\right)=0$, d'où $\tilde{u}(T)$ défini comme dans le lemme 1 satisfait $\tilde{u}(T) \geq u(T)+2$. Donc, l'écart dans le théorème 6 satisfait $d_{f_{\alpha}, a} \geq 2$ et

$$
N_{0}^{\prime}(T)=\tilde{u}(T) \geq u(T)+2=\frac{1}{\pi} \arg h\left(\frac{1}{2}+i T\right)+\frac{3}{2},
$$

puis

$$
0 \leq N(T)-N_{0}^{\prime}(T) \leq \frac{1}{2}
$$

d'où $N(T)=N_{0}(T)=N_{0}^{\prime}(T)$ et tous les zéros de la fonction

$$
\hat{f}_{3 / 5}(s)=\zeta^{*}\left(s+\frac{3}{5}\right)+\zeta^{*}\left(s-\frac{3}{5}\right)
$$

sont sur la droite $\sigma=\frac{1}{2}$ et ils sont simples. 
Pourtant, pour des grandes valeurs de $\alpha$, l'argument de $h_{\alpha}(a+i \tau)$ est une fonction croissante de $\tau>0$, et il est impossible d'améliorer (17) avec nos estimations. Si on prend $\alpha$ sufisamment grand, par exemple $\alpha=8$, la fonction $\hat{f}_{8}(s)=\zeta^{*}(s+8)+\zeta^{*}(s-8)$ a un zéro en $s_{0} \approx 8.78369+1.00496 i$, à l'exterieur de la droite critique, et un autre symétrique $1-\overline{s_{0}}$ dans le demi-plan supérieur. Donc $N(T)-N_{0}(T)=2$, et l'estimation (17) est optimale. On conclut qu'il y a exactement 4 zéros hors la droite critique dans ce cas, tous simples. En plus, $N_{0}(T)=N_{0}^{\prime}(T)$, donc tous les zéros de $\hat{f}_{8}(s)$ sur la droite critique sont simples.

Il y a un zéro double de $f_{\alpha}(s)$ pour $\alpha \approx 2,61117$, en $s_{0}=\frac{1}{2}+i \tau_{0} \approx \frac{1}{2}+5,4983 i$. Ceci peut être détecté facilement en remarquant que le zéro $\frac{1}{2}+i \tau_{0}$ de $f_{\alpha}(s)$ est un zéro double si et seulement si $u_{\alpha}^{\prime}\left(\tau_{0}\right)=0$, où

$$
u_{\alpha}(\tau)=\frac{1}{\pi} \arg h_{\alpha}\left(\frac{1}{2}+i \tau\right)-\frac{1}{2} .
$$

Dans notre cas, cela correspond à un minimum de $u_{\alpha}(\tau)$.

On peut aussi faire l'analyse de $f_{\alpha}(s)$ sous l'hypothèse de Riemann pour $0<\alpha<\frac{1}{2}$.

Proposition 13. Soit $0<\alpha<\frac{1}{2}$. Sous l'hypothèse de Riemann, tous les zéros non réels de

$$
f_{\alpha}(s)=\zeta^{*}(s+\alpha)-\zeta^{*}(s-\alpha)
$$

sont sur la droite $\sigma=\frac{1}{2}$ et ils sont simples. Cette fonction a exactement trois zéros réels simples $s=\frac{1}{2}, s=\rho_{\alpha}$ et $s=1-\rho_{\alpha}$, ò̀ $\left.\rho_{\alpha} \in\right] 1+\alpha,+\infty[$.

Démonstration. En effet, $n_{h, \sigma>\frac{1}{2}}=0$ et $p_{h, \sigma>\frac{1}{2}}=1$ (on compte le pôle $s=1-\alpha$ de $h_{\alpha}(s)$ ); de plus, $h_{\alpha}(s)$ ne s'annulle pas pour $\sigma \geq \frac{1}{2}$ sous l'hypothèse de Riemann, et

$$
\frac{1}{\zeta(s+\alpha)}=O(\log |\tau|)
$$

uniformément pour $\sigma \geq \frac{1}{2}, \alpha>0$ fixé [33, Theorem 14.2]). Donc

$$
N(T)-N_{0}(T) \leq N(T)-N_{0}^{\prime}(T) \leq \frac{1}{2},
$$

d'où tous les zéros non réels de $f(s)$ satisfont $\sigma=\frac{1}{2}$ et ils sont simples.

Pour obtenir des résultats inconditionnels pour $0<\alpha<1 / 2$, il y a deux ingrédients importants à fournir : d'un côté, une borne de $\zeta(s)^{-1}$ pour $\sigma \geq 1 / 2$, différente de (18); d'un autre côté, une estimation pour le nombre de zéros de la fonction zêta dans le demi-plan $\sigma \geq 1 / 2+\alpha$, supposer qu'on a un nombre fini de zéros dans un tel demi-plan est une condition trop forte (cela entraîne l'existence d'un demi-plan $\sigma \geq \beta>1 / 2$ libre de zéros de la fonction zêta). On dévéloppera ces outils aux $\S \S 8,9$,

Les fonctions $f_{\alpha}^{ \pm}(s)=\zeta^{*}(s+\alpha) \pm \zeta^{*}(s-\alpha)$ ont été récemment étudiées par H. Ki dans [15]. La méthode de Titchmarsh dans $[34, \S \S 9.3,9.4]$ donne la formule pour le nombre de zéros de $f_{\alpha}^{ \pm}(s)$ avec $0<\tau<T$

$$
N(T)=\frac{T}{2 \pi} \log \frac{T}{2 \pi}-\frac{T}{2 \pi}+O(\log T),
$$

où la constante dans $O(\cdot)$ dépend de $\alpha>0$ (la formule dans [15, Proposition 2.2] pour $N(T)$ explicite la dépendance de $\alpha$ ).

Comme il est naturel, $\mathrm{H}$. Ki étudie séparément les cas $0<\alpha<1 / 2$ et $\alpha \geq 1 / 2$. On remarque une faute dans son article, dans l'étude du cas $\alpha \geq 1 / 2$, pour confirmer que ses résultats sont cohérents avec les notres. H. Ki ne s'aperçoit pas de l'existence d'un zéro réel de $f_{\alpha}^{-}(s)$ (trouvé dejà par P. R. Taylor dans la preuve de la proposition 12), ni des zéros non réels et hors la droite critique de $f_{\alpha}^{+}(s)$. Dans les dernières lignes de son article, il montre que la fonction

$$
(s+\alpha)(s+\alpha-1)(s-\alpha)(s-\alpha-1) f_{\alpha}(s)
$$


a au plus 4 zéros hors la droite critique (ce qui coïncide avec notre résultat), puis il factorise le facteur $(s+\alpha)(s+\alpha-1)(s-\alpha)(s-\alpha-1)$ en considérant qu'il contient exactement 4 zéros, pour conclure que tous les zéros de $f_{\alpha}(s)$ sont sur la droite critique. Ce dernier raisonnement est erroné, vu que ces facteurs correspondent aux pôles de $f_{\alpha}(s)$, et donc ne peuvent pas être comptés commes des zéros de la fonction. La simplicité des zéros n'est pas traitée.

Pour $0<\alpha<1 / 2$, H. Ki localise les zéros de $f_{\alpha}^{ \pm}(s)$ dans une région qui dépend des possibles zéros de la fonction zêta dans le demi-plan $\sigma>1 / 2+\alpha$ [15, Theorem B]; il étudie aussi les conséquences de l'hypothèse de Lindelöf sur cette région [15, Theorem C]. Cela implique un résultat de densité zéro pour les zéros hors la droite $\sigma=1 / 2$, dont on en parlera au §9.

On peut modifier la première fonction considérée et obtenir des résultats similaires. On prend $h_{\alpha}(s)=\xi(s+\alpha)$ avec $\alpha>0$, la fonction $h_{\alpha}(s)$ est entière et on peut appliquer le corollaire 5. Les estimations asymptotiques de $\xi(s+\alpha)$ sont les mêmes que celles de $\zeta^{*}(s+\alpha)$, le facteur $\frac{1}{2}(s+\alpha)(s+\alpha-1)$ ne change pas le comportement de la fonction. L'absence de pôles de cette fonction simplifie les résultats.

Les fonctions $\tilde{f}_{\alpha}(s)=\xi(s+\alpha) \pm \xi(s-\alpha)$, ont donc tous leurs zéros simples, alignés sur la droite critique $\sigma=\frac{1}{2}$, pour $\alpha \geq \frac{1}{2}$ inconditionnellement, et sous l'hypothèse de Riemann pour $0<\alpha<\frac{1}{2}$ (une autre preuve de ce résultat peut être vue dans l'annexe A). Cette fonction a été étudiée par Lagarias dans [19], qui donne aussi l'estimation correspondante de $N(T)$. Lagarias étudie aussi la distribution limite des espacements entre les zéros consecutifs des fonctions $\tilde{f}_{\alpha}(s)$, et la relation avec la conjecture GUE pour la fonction zêta de Riemann.

\section{Infinité de zéros sur la droite critique}

L'existence d'une quantité finie de zéros de la fonction $h(s)$ sur la droite $\sigma=a$ n'est pas une barrière pour l'application du théorème 4 (remarque (a) au théorème) et ne change pas le résultat du théorème 4. On suppose maintenant qu'il y a une infinité de zéros sur la droite critique $\sigma=a$. Il est évident qu'on ne peut plus utiliser le lemme 2 pour essayer d'étendre le théorème 4 . On obtient la généralisation naturelle de ce lemme avec la technique de N. Levinson dans [22].

Introduisons d'abord quelques notations. Pour une fonction $h(s)$ et $\sigma_{0}<\sigma_{1}, T>0$, on note

$$
N_{h}\left(\sigma_{0}, \sigma_{1}, T\right)=\#\left\{s \in \mathbb{C} \mid h(s)=0, \sigma_{0} \leq \sigma \leq \sigma_{1}, 0<\tau<T\right\},
$$

et $N_{h}\left(\sigma_{0}, T\right)=N_{h}\left(\sigma_{0},+\infty, T\right)$.

Lemme 14. Soit $a \in \mathbb{R}, h(s)$ une fonction méromorphe, réelle sur la droite réelle, avec un nombre fini de pôles, $h(a) \neq 0$, holomorphe sur la droite $\sigma=a$, et holomorphe et non nulle sur la droite $\sigma=\sigma_{0}, \sigma_{0}>a$. On définit, pour $T>0, R\left(\sigma_{0}, T\right)$ par

$$
R\left(\sigma_{0}, T\right)=\frac{1}{\pi} \Delta \arg h(s)
$$

où $\Delta$ indique la variation d'argument de $h(s)$, obtenue par variation continue le long des segments qui joignent (dans l'ordre indiqué) $\sigma_{0}, \sigma_{0}+i T$ et $a+i T$. On dénote par $n_{h, a<\sigma<\sigma_{0}}$ le nombre de zéros réels de $h(s)$ dans l'intervalle $a<\sigma<\sigma_{0}$ et par $P_{h, a<\sigma<\sigma_{0}}$ le nombre de pôles de $h(s)$ avec $a<\sigma<\sigma_{0}$.

Le nombre de zéros de $f(s)$ sur la droite critique $\sigma=a$ avec $0<\tau<T$ est minoré par

$$
N_{0}^{\prime}(T) \geq R\left(\sigma_{0}, T\right)+\left(-n_{h, a<\sigma<\sigma_{0}}+P_{h, a<\sigma<\sigma_{0}}\right)-2 N_{h}\left(a, \sigma_{0}, T\right)-2 .
$$

Remarque. Si les zéros de $h(s)$ sont restreints à une bande finie $|\sigma-a|<\sigma_{0}$, avec les notations du théorème 4, (19) devient

$$
N_{0}^{\prime}(T) \geq R(T)+\left(-n_{h, \sigma>a}+P_{h, \sigma>a}\right)-2 N_{h}(a, T)-2 .
$$


Démonstration. Soit $T>0$ fixé tel que $h(s)$ n'a pas de zéro sur la droite $\tau=T$, et $a_{1}, a_{2}, \ldots, a_{M}$ les différents zéros de $h(s)$ sur le segment qui joint $a$ et $a+i T: \Re\left(a_{i}\right)=a$ et

$$
0<\Im\left(a_{1}\right)<\Im\left(a_{2}\right)<\cdots<\Im\left(a_{M}\right)<T .
$$

On considère le rectangle modifié $C_{\varepsilon}$, formé par déformation du rectangle

$$
C: \quad a \leq \sigma \leq \sigma_{0}, \quad 0 \leq \tau \leq T
$$

par des demi-cercles de rayon $\varepsilon>0$ qui laissent à l'extérieur les zéros et les pôles de $h(s)$ sur la frontière du rectangle non modifié $C$.

Sur ce rectangle, en partant de la valeur en $s=\sigma_{0}$, l'argument de $h(s)$ est bien défini. On pose les quantités

$$
\begin{aligned}
& H_{0}=\arg h\left(a+i\left(a_{1}-0\right)\right)-\arg h(a) \\
& H_{j}=\arg h\left(a+i\left(a_{j+1}-0\right)\right)-\arg h\left(a+i\left(a_{j}+0\right)\right), \quad 1 \leq j \leq M-1, \\
& H_{M}=\arg h(a+i T)-\arg h\left(a+i\left(a_{M}+0\right)\right) .
\end{aligned}
$$

En appliquant le principe de l'argument sur $C_{\varepsilon}$ et en prenant la limite lorsque $\varepsilon \rightarrow 0^{+}$on obtient

$$
\begin{aligned}
& \pi\left(-n_{h, a<\sigma<\sigma_{0}}+p_{h, a<\sigma<\sigma_{0}}\right)+\pi R\left(\sigma_{0}, T\right)-\sum_{j=0}^{M} H_{j} \\
& \quad-\pi\left(N_{h}\left(a, \sigma_{0}, T\right)-N_{h}\left(a+0, \sigma_{0}, T\right)\right)=2 \pi\left(N_{h}\left(a+0, \sigma_{0}, T\right)-p_{h, a<\sigma<\sigma_{0}}^{\prime}\right),
\end{aligned}
$$

où $p_{h, a<\sigma<\sigma_{0}}$ est le nombre de pôles réels, $p_{h, a<\sigma<\sigma_{0}}^{\prime}$ le nombre de pôles non réels de partie imaginaire strictement positive de $h(s)$ avec $a<\sigma<\sigma_{0}$, de façon que $p_{h, a<\sigma<\sigma_{0}}+2 p_{h, a<\sigma<\sigma_{0}}^{\prime}=P_{h, a<\sigma<\sigma_{0}}$.

On réécrit la formule comme

$$
\begin{aligned}
\sum_{j=0}^{M} H_{j}= & \pi\left(-n_{h, a<\sigma<\sigma_{0}}+P_{h, a<\sigma<\sigma_{0}}\right)+\pi R\left(\sigma_{0}, T\right) \\
& \pi\left(N_{h}\left(a, \sigma_{0}, T\right)-N_{h}\left(a+0, \sigma_{0}, T\right)\right)-2 \pi N_{h}\left(a, \sigma_{0}, T\right) .
\end{aligned}
$$

Dans chaque intervalle (ouvert) correspondant à $H_{j}$, il y a au moins $H_{j} / \pi-2$ zéros de $f(s)$ (par un analogue au lemme 2), ce qui rajouté aux $M$ zéros connus de $f(s)$ (les zéros de $h(s)$ sur la droite critique), nous donne

$$
\begin{aligned}
N_{0}^{\prime}(T) \geq & M+\sum_{j=0}^{M}\left(\frac{H_{j}}{\pi}-2\right)=-M-2+\frac{1}{\pi} \sum_{j=0}^{M} H_{j} \\
= & -M-2+\left(-n_{h, a<\sigma<\sigma_{0}}+P_{h, a<\sigma<\sigma_{0}}\right)+R\left(\sigma_{0}, T\right) \\
& +\left(N_{h}\left(a, \sigma_{0}, T\right)-N_{h}\left(a+0, \sigma_{0}, T\right)\right)-2 N_{h}\left(a, \sigma_{0}, T\right) .
\end{aligned}
$$

Ceci avec l'estimation

$$
N_{h}\left(a, \sigma_{0}, T\right)-N_{h}\left(a+0, \sigma_{0}, T\right) \geq M
$$

nous donne le résultat attendu.

\section{Infinité de zéros à droite de la droite critique et théorèmes de densité}

Considérons pour l'instant les translatées de la fonction zêta de Riemann étudiées dans le $§ 7$. L'étude des fonctions telles que $f_{\alpha}(s)=\zeta^{*}(s+\alpha) \pm \zeta^{*}(s-\alpha)$ pour $0<\alpha<1 / 2$ avec le théorème 4 est possible sous l'hypothèse de Riemann ou sous l'hypothèse de finitude du nombre de zéros 
de la fonction zêta dans le demi-plan $\sigma \geq \frac{1}{2}+\alpha$, ce qui est une condition trop forte à demander. Le meilleur qu'on a ce sont les résultats de densité zéro, c'est-à-dire, des estimations de type $N_{\zeta}(\beta, T)=O\left(T^{\theta}\right), 0 \leq \theta<1$ pour le nombre de zéros de la fonction zêta avec $\sigma \geq \beta, 0<\tau<T$, lorsque $\beta>\frac{1}{2}$. Mais un résultat de ce type indique qu'un $0 \%$ des zéros de la fonction $\zeta^{*}(s+\alpha)$ sont à droite de la droite critique $\sigma=1 / 2$. L'analogue du théorème 4 devrait s'énoncer comme suit : « un $0 \%$ des zéros de $f_{\alpha}(s)$ sont en dehors de la droite critique $\sigma=1 / 2 »$. En particulier, le $100 \%$ des zéros de $f_{\alpha}(s)$ devraient être sur la droite $\sigma=1 / 2$ !

On revient au cas général. Pour simplifier la notation, nous allons étudier seulement les fonctions $f(s)=h(s) \pm h(2 a-s)$ dont la fonction $h(s)$ a les zéros inclus dans une bande $|\sigma-a|<\sigma_{0}$.

Théorème 15. Soit $h(s)$ une fonction méromorphe sur $\mathbb{C}$, réelle sur la droite réelle, qui ne s'annulle pas pour $\sigma$ assez grand, avec un nombre fini de pôles. Avec les notations du théorème 4 , que la fonction $F(s)$ satisfait la condition (i), et

(ii') il existe une fonction croissante $\phi: \mathbb{R} \rightarrow \mathbb{R}$ telle que, pour chaque $\sigma_{0}>$ a, il existe une constante $K>0$ et une suite $\left(T_{n}\right)_{n}$ telles que $\lim _{n \rightarrow \infty} T_{n}=+\infty$,

$$
T_{n} \leq T_{n+1} \leq \phi\left(T_{n}\right) \text { pour } n \geq 1
$$

et

$$
|F(s)|<e^{K|s|} \text { pour } a \leq \sigma \leq \sigma_{0},|\tau|=T_{n}, n \geq 1
$$

Alors, pour $T>0$

$$
N(T)-N_{0}^{\prime}(T) \leq 4 N_{h}(a, \phi(2 T))+O(1) .
$$

Démonstration. On suit la démarche dans la démonstration du théorème 4. Sans perte de généralité, on peut supposer $h(s)$ holomorphe sur la droite critique $\sigma=a$ (remarque (a) au théorème 4). Soit $\sigma_{0}$ assez grand pour satisfaire (i) avec $\varepsilon=\frac{1}{2}$, au même temps que la bande $2 a-\sigma_{0} \leq \sigma \leq \sigma_{0}$ contient tous les zéros et pôles de $h(s)$ et $f(s)$. D'un autre côté, on prend $K>0$ et la suite $\left(T_{n}\right)_{n}$ associés à $\sigma_{0}$ en (ii $\left.{ }^{\prime}\right)$.

On calcule la variation d'argument de $f(s)$ le long du rectangle $R_{n}$ défini par

$$
-T_{n} \leq \tau \leq T_{n}, \quad 2 a-\sigma_{0} \leq \sigma \leq \sigma_{0} .
$$

Le théorème de Littlewood nous donne la formule (7), d'où l'on déduit que

$$
\pi \int_{0}^{T_{n}} S(\tau) d \tau \leq \sigma_{0} K\left(\sigma_{0}+T_{n}\right)+\frac{1}{2} T_{n}-I\left(\sigma_{0}\right)
$$

Donc, il existe $C=C\left(\sigma_{0}\right)>0$ tel que

$$
\frac{1}{T_{n}} \int_{0}^{T_{n}} S(\tau) d \tau \leq C
$$

pour $n$ assez grand, et pas forcément une borne inférieure, sinon on mettrait $O(1)$ au lieu de la borne $C$.

D'un autre côté, le lemme 2 est remplacé par le lemme 14, ce qui nous donne l'estimation (20)

$$
N_{0}^{\prime}(T) \geq R(T)-2 N_{h}(a, T)+O(1) .
$$

Puis, pour $0<T^{\prime}<T$

$$
\begin{aligned}
\left(T-T^{\prime}\right)\left(N\left(T^{\prime}\right)-N_{0}^{\prime}\left(T^{\prime}\right)\right) & \leq \int_{0}^{T}\left(N(\tau)-N_{0}^{\prime}(\tau)\right) d \tau \\
& \leq \int_{0}^{T} 2 N(a, \tau) d \tau+\int_{0}^{T} S(\tau) d \tau+O(T)
\end{aligned}
$$


On prend $T=T_{n}, T^{\prime}=T_{n} / 2$ pour obtenir

$$
\frac{T_{n}}{2}\left(N\left(T_{n} / 2\right)-N_{0}^{\prime}\left(T_{n} / 2\right)\right) \leq 2 N_{h}\left(a, T_{n}\right) T_{n}+\int_{0}^{T_{n}} S(\tau) d \tau+O\left(T_{n}\right) .
$$

En divisant par $T_{n} / 2$ et par (21)

$$
N\left(T_{n} / 2\right)-N_{0}^{\prime}\left(T_{n} / 2\right) \leq 4 N_{h}\left(a, T_{n}\right)+O(1) .
$$

Cette fois-ci on a fait disparaître la constante $C$ vu qu'on traite des quantités positives. Soit maintenant $T>0$ suffisamment grand. Alors, il existe $n \geq 1$ tel que $T_{n-1} / 2 \leq T \leq T_{n} / 2$, d'où

$$
T_{n} \leq \phi\left(T_{n-1}\right) \leq \phi(2 T) .
$$

La croissance des fonctions $N(T)-N_{0}^{\prime}(T)$ et $N_{h}(a, T)$ (par rapport à $T$ ) nous permettent de conclure.

\section{Remarques.}

1. La condition (i) du théorème 4 apliquée est beaucoup plus forte de ce dont on a besoin, elle peut être remplacé par une condition plus faible, comme dans le théorème 24.

2. Dans la dernière démonstration on évite de prendre la limite lorsque $T \rightarrow+\infty$; ce qui est claire si l'on suppose que $\lim _{T \rightarrow \infty} N_{h}(a, T)=+\infty$ (sinon on tombe dans le théorème 4 ). Ceci explique aussi le terme d'erreur $O(1)$. L'interêt du résultat est le fait de pouvoir calculer l'ordre de croissance du nombre de zéros de $f(s)$ hors la droite critique $\sigma=a$.

3. Si $N(a, T)=O\left(T^{A} \log ^{B} T\right)$ et on peut choisir $\phi(T)$ linéaire en $T$, alors

$$
N(a, \phi(T))=O\left(T^{A} \log ^{B} T\right) .
$$

On reprend l'analyse des traslatées de la fonction zêta de Riemann. On rappelle pour cela des propriétés de la fonction zêta :

Lemme 16. Pour la fonction zêta de Riemann $\zeta(s)$, on a :

1. Pour $\frac{1}{2} \leq \sigma \leq 1$ [13, p. 275]

$$
N_{\zeta}(\sigma, T)=\left\{\begin{array}{cl}
O\left(T^{3(1-\sigma) /(2-\sigma)} \log ^{5} T\right), & \frac{1}{2} \leq \sigma \leq \frac{3}{4} \text { (Ingham) }, \\
O\left(T^{3(1-\sigma) /(3 \sigma-1)} \log ^{44} T\right), & \frac{3}{4} \leq \sigma \leq 1 \text { (Huxley) }
\end{array}\right.
$$

2. Il existe une constante $A>0$ telle que pour chaque $n \geq 1$, il existe $n<T_{n}<n+1$ tel que

$$
|\zeta(s)|>\tau^{-A}
$$

lorsque $\tau=T_{n},-1 \leq \sigma \leq 2[34, \S 9.7]$.

Proposition 17. Soit $0<\alpha<1 / 2$. Pour la fonction $f_{\alpha}(s)=\zeta^{*}(s+\alpha) \pm \zeta^{*}(s-\alpha)$, on a

$$
N(T)-N_{0}^{\prime}(T) \leq 4 N_{\zeta}\left(\frac{1}{2}+\alpha, 2 T+4\right)+O(1) .
$$

En particulier,

$$
N(T)-N_{0}^{\prime}(T)= \begin{cases}O\left(T^{3(1-2 \alpha) /(3-2 \alpha)} \log ^{5} T\right), & 0<\alpha \leq \frac{1}{4} \\ O\left(T^{3(1-2 \alpha) /(1+6 \alpha)} \log ^{44} T\right), & \frac{1}{4} \leq \alpha \leq \frac{1}{2}\end{cases}
$$

Démonstration. La condition (i) du théorème 4 est toujours satisfaite, mais pas la condition (iii). On combine le lemme 16 (2), et les estimations utilisées dans l'étude du cas $\alpha \geq \frac{1}{2}$ dans 
l'équation (15), pour obtenir une suite $\left(T_{n}\right)$, avec $n<T_{n}<n+1$ et une constante $B>0$ telle que la fonction $F_{\alpha}(s)=\zeta^{*}(s-\alpha) / \zeta^{*}(s+\alpha)$ satisfait

$$
F_{\alpha}(s)=O\left(\tau^{B}\right)
$$

pour $\sigma \geq \frac{1}{2}, \tau=T_{n}, n \geq 1$. En prenant $\phi(T)=T+2$, on a la condition (ii $)$ du théorème 15. Cela nous donne le résultat principal. Les estimations particulières sont conséquence d'appliquer celles de Ingham et Huxley dans le lemme 16 (1).

Le résultat obtenu entraîne une densité zéro pour les zéros des fonctions $f_{\alpha}(s)$ hors la droite critique $\sigma=\frac{1}{2}$. On peut comparer ce résultat avec celui de H. Ki [15, Theorem B], dont on a parlé à la fin du §7, qui entraîne

$$
N(T)-N_{0}(T)=O\left(N_{\zeta}\left(\frac{1}{2}+\alpha+0, T\right) \log T\right) .
$$

Les estimations obtenues restent les mêmes pour les fonctions $f(s)=\xi(s+\alpha) \pm \xi(s-\alpha), 0<\alpha<\frac{1}{2}$.

\section{Intégrales associées à des séries d'Eisenstein}

La série d'Eisenstein non-holomorphe $E(z, s)$ pour le groupe modulaire $\operatorname{PSL}(2, \mathbb{Z})$, pour $z=$ $x+i y$ dans le demi-plan supérieur $\mathbb{H}=\{z \in \mathbb{C} \mid y>0\}$ est définie par

$$
E(z, s)=\frac{1}{2} \sum_{(m, n) \neq(0,0)} \frac{y^{s}}{|m z+n|^{2 s}},
$$

pour $\sigma>1$. Cette série admet un prolongement analytique dans le plan complexe, sauf aux points $s=0, s=1$ où elle a des pôles simples, avec résidu $\operatorname{Res}_{s=1} E(z, s)=3 / \pi$, et satisfait à l'équation fonctionnelle

$$
E^{*}(z, s)=E^{*}(z, 1-s)
$$

où $E^{*}(z, s)=\pi^{-s} \Gamma(s) E(z, s)$ est la série d'Eisenstein complétée. Cette série contient la fonction zêta de Riemann; en fait $E(i, s)=2 \zeta(s) L\left(s, \chi_{-4}\right)$, où $\chi_{-4}(n)=(-4 / n)$; donc on peut attendre, dans certains cas, une hypothèse de Riemann pour $E(z, s)$.

La série d'Eisenstein agit comme une forme modulaire de poids zéro, puisque

$$
E\left(\frac{a z+b}{c z+d}, s\right)=E(z, s) \text { pour }\left[\begin{array}{ll}
a & b \\
c & d
\end{array}\right] \in \operatorname{PSL}(2, \mathbb{Z}) .
$$

En particulier, $E(z+1, s)=E(z, s)$, et par la périodicité par rapport à la variable $x$, on a un dévéloppement de Fourier de la série, la formule de Chowla-Selberg [29, §1] :

$$
\begin{aligned}
E(z, s)= & \zeta(2 s) y^{s}+\sqrt{\pi} \frac{\Gamma(s-1 / 2)}{\Gamma(s)} \zeta(2 s-1) \\
& +2 \sqrt{y} \frac{\pi^{s}}{\Gamma(s)} \sum_{n=1}^{\infty} n^{1 / 2-s} \sum_{d \mid n} d^{2 s-1} \int_{-\infty}^{+\infty} e^{-2 \pi n y \cosh \tau} e^{(s-1 / 2) \tau} d \tau \cdot \cos (2 \pi n x) .
\end{aligned}
$$

Pour $A>0$ et $s \in \mathbb{C}$, l'intégrale

$$
2 K_{s}(2 A)=\int_{-\infty}^{+\infty} e^{-2 A \cosh \tau} e^{s \tau} d \tau
$$

est une fonction de Bessel. Elle est une fonction entière de $s$, et satisfait aux équations (cf. [24, p.307])

$$
s K_{s}(2 A)=A\left(K_{1+s}(2 A)-K_{1-s}(2 A)\right), \quad K_{s}(2 A)=K_{-s}(2 A) \text { pour } s \in \mathbb{C} .
$$


Ces équations montrent que la fonction de Bessel correspond bien à un cas particulier des fonctions en étude dans notre travail (plus précisement, au théorème 40). Pólya a montré que tous les zéros de la fonction de Bessel sont simples, et sur la droite $\sigma=0$ [24, §VI]. La fonction diviseur

$$
\sigma_{s}(n)=\sum_{d \mid n} d^{s}=\prod_{p^{e} \| n} \frac{1-p^{(e+1) s}}{1-p^{s}}
$$

est une fonction entière, aussi avec tous leurs zéros sur la droite $\sigma=0$ et simples. Donc, les coefficients non-nuls et non-constants dans la série de Fourier de $E(z, s)$ ont tous leurs zéros sur la droite $\sigma=\frac{1}{2}$.

Par contre, le terme constant du dévéloppement de Fourier de $E^{*}(z, s)$

$$
a_{0}(y, s)=\int_{0}^{1} E^{*}(x+i y) d x=\zeta^{*}(2 s) y^{s}+\zeta^{*}(2-2 s) y^{1-s}
$$

peut avoir deux zéros hors la droite critique, (corollaire 21), mais qu'en nombre fini, tous les autres restant sur cette droite et simples. La répartition des zéros de cette fonction a été étudiée par Hejhal [11], Suzuki et Lagarias [20], et finalement H. Ki [18].

Plus généralement, on peut considérer une intégrale de $E^{*}(z, s)$ sur la surface modulaire $\operatorname{PSL}(2, \mathbb{Z}) \backslash \mathbb{H}$ par rapport à une mésure positive, tel que la mésure hyperbolique $d \mu(z)=y^{-2} d x d y$

$$
\int_{\mathscr{D}} E^{*}(z, s) d \mu(z)
$$

où $\mathscr{D}$ est le domaine fondamental $\mathscr{D}=\left\{z \in \mathbb{H}|| z|\geq 1| x \mid, \leq \frac{1}{2}\right\}$. Des telles intégrales aparaissent dans le calcul des intégrales des fonctions automorphes par la méthode de Rankin-Selberg. Soit $F(z)$ une fonction continue sur $\mathbb{H}$, invariante par $\operatorname{PSL}(2, \mathbb{Z})$, dont le terme constant dans le dévéloppement de Fourier est $b_{0}(y)=\int_{0}^{1} F(x+i y) d x$. L'intégrale de $F(z)$ sur $\mathscr{D}$ peut être récupérée par le calcul du résidu (sous les bonnes conditions de convergence)

$$
\frac{3}{\pi} \int_{\mathscr{D}} F(z) d \mu(z)=\operatorname{Res}_{s=1} \int_{\mathscr{D}} F(z) E(z, s) \mu(z) .
$$

En fait,

$$
\int_{\mathscr{D}} F(z) E(z, s) \mu(z)=\int_{0}^{\infty} b_{0}(y) y^{s-2} d y=R(F, s)
$$

(cf. [37, p. 415]), ce qui réduit le calcul d'intégration sur le domaine fondamental à l'intégration sur une droite. La fonction $R(F, s)$ est appelé la transformée de Rankin-Selberg de $F(z)$; elle est une fonction méromorphe, et satisfait à l'équation fonctionnelle $R^{*}(F, s)=R^{*}(F, 1-s)$, où $R^{*}(F, s)=$ $\zeta^{*}(2 s) R(F, s)$. Pour contourner les problèmes de convergence qu'on rencontre dans la pratique, Zagier introduit dans [37] une procédure de renormalisation d'intégrales, par l'introduction des domaines tronqués $\mathscr{D}_{T}=\left\{z \in \mathbb{H}|| z|\geq 1| x \mid, \leq \frac{1}{2}, y \leq T\right\}$. Comme partie des résultats de Zagier, on retrouve une formule de $R^{*}(F, s)$ incluant un terme de la forme

$$
H(y ; s)=p(s) \zeta^{*}(2 s) y^{s}+p(1-s) \zeta^{*}(2-2 s) y^{1-s},
$$

où $p(s)$ est une fonction rationnelle [37, p. 419].

Certaines de ces intégrales ont attiré l'attention ces derniers temps. Lin Weng a introduit récemment des fonctions zêta de rang $n$ non abéliennes, associées à des corps de nombres, en analogie à l'intégrale d'Iwasawa-Tate, dans [36]. On particularise l'étude au corps des nombres rationnels. La fonction zêta de Weng de rang 2 correspond à l'intégration de la série d'Eisenstein complétée sur un ensemble de réseaux, les réseaux semi-stables de $\mathbb{R}^{2}$. Un réseau $L=L(z)=$ 
$\{m z+n \mid m, n \in \mathbb{Z}\}, z \in \mathscr{D}$ est semi-stable si, et seulement si $z \in \mathscr{D}_{1}$ [9, Example 1.25], et la fonction zêta de Weng est

$$
Z_{2, \mathbb{Q}}(s)=\int_{\mathscr{D}_{1}} E^{*}(z, s) d \mu(z) .
$$

Suivant la renormalisation de Zagier, on considère les intégrales, pour $T \geq 1$, données par

$$
Z_{2, \mathbb{Q}}^{T}(s)=\int_{\mathscr{D}_{T}} E^{*}(z, s) d \mu(z) .
$$

La méthode de Rankin-Selberg nous donne alors des formules closes pour les fonctions zêta de Weng (cf. [37, pp. 426-427], [20, §1]) :

$$
Z_{2, \mathbb{Q}}^{T}(s)=\zeta^{*}(2 s) \frac{T^{s-1}}{s-1}-\zeta^{*}(2-2 s) \frac{T^{-s}}{s},
$$

et en particulier

$$
Z_{2, \mathbb{Q}}(s)=\zeta^{*}(2 s) \frac{1}{s-1}-\zeta^{*}(2-2 s) \frac{1}{s} .
$$

On veut maintenant étudier la répartition des zéros de ces fonctions d'une façon unifiée. Les fonctions zêta de Weng et le terme constant de la zérie d'Eisenstein sont des cas particuliers de la formule (24). Lagarias et Suzuki proposent dans [20] l'étude de cette fonction pour $y \geq 1$, et $p(s)$ est un polynôme à coefficients réels, ce qui équivaut à l'étude avec une fonction rationnelle $p(s)$.

On fait d'abord quelques remarques sur les racines de $p(s)$. Par la remarque (a) du théorème 4, on peut supposer que $s=\frac{1}{2}$ est une racine simple de $p(s)$, et qu'il n'y a pas d'autres zéros pour $p(s)$ sur la droite critique $\sigma=\frac{1}{2}$, parce que ces zéros sont aussi des zéros de $H(y, s)$ de la même multiplicité, et qui peuvent être facilement factorisés. Il faut aussi voir que $H(y ; s)$ a un pôle en $s=1$ si et seulement si $p(0) \neq 0$. L'application du théorème 4 et le lemme 7 nous fournissent le résultat suivant.

Théorème 18. Soit

$$
H^{ \pm}(y ; s)=p(s) \zeta^{*}(2 s) y^{s} \pm p(1-s) \zeta^{*}(2-2 s) y^{1-s},
$$

où $y \geq 1, p(s)$ un polynôme à coefficients réels, tel que $p(s)=(2 s-1) q(s)$, où $q(s)$ est un polynôme sans racine sur $\sigma=\frac{1}{2}$. Alors

(i) Le nombre de zéros non réels et de partie imaginaire positive de $H^{ \pm}(y ; s)$ en dehors de la droite critique $\sigma=\frac{1}{2}$ est borné par

$$
N(T)-N_{0}(T) \leq N(T)-N_{0}^{\prime}(T) \leq u_{ \pm}-n_{H, \sigma>\frac{1}{2}}-\frac{n_{H, \frac{1}{2}}}{2}+\chi_{H, 0}+N_{p, \sigma>\frac{1}{2}}-d_{H, \frac{1}{2}},
$$

où $n_{H, \sigma>\frac{1}{2}}$ note le nombre de zéros réels de $H^{ \pm}(y ; s)$ avec $\sigma>\frac{1}{2}, n_{H, \frac{1}{2}}$ la multiplicité de $s=\frac{1}{2}$ comme zéro de $H^{ \pm}(y ; s)$ (0 pour $H^{+}(y ; s), \geq 1$ et impaire pour $\left.H^{-}(y ; s)\right), \chi_{H, 0}=1$ si $p(0) \neq 0$ et $\chi_{H, 0}=0$ si $p(0)=0, N_{p, \sigma>\frac{1}{2}}$ est le nombre de racines de $p(s)$ avec $\sigma>\frac{1}{2}$, et $d_{H, \frac{1}{2}} \geq 0$ est le nombre entier dans le théorème 6 (équation (11)).

(ii) En particulier, si $s=0$ est un zéro de $p(s)$, et le seul zéro de $p(s)$ avec $\sigma \geq \frac{1}{2}$ est $s=\frac{1}{2}$, alors tous les zéros de $H^{ \pm}(y ; s)$ sont sur la droite critique $\sigma=\frac{1}{2}$, et ils sont simples.

(iii) Soit $y^{*}=4 \pi e^{-\gamma-q^{\prime}\left(\frac{1}{2}\right) / q\left(\frac{1}{2}\right)}$. Alors, pour la fonction $H^{-}(y ; s)$,

(a) Si $y<y^{*}$, on a $d_{H, \frac{1}{2}} \geq 1$;

(b) Si $y=y^{*}$, la multiplicité de $s=\frac{1}{2}$ comme racine de $H^{-}(y ; s)$ est $\geq 3$;

(c) Si $y>y^{*}$, il y a un zéro $\rho$ de $H^{-}(y ; s)$ dans l'intervalle $] \frac{1}{2}, 1\left[\left(\right.\right.$ et $1-\rho$ dans $\left.\left(0, \frac{1}{2}\right)\right)$ si $q\left(\frac{1}{2}\right) q(0)>0\left(q\left(\frac{1}{2}\right)\right.$ et $q(0)$ ont le même signe), donc $n_{H, \sigma>\frac{1}{2}} \geq 1$. 
Remarque. La borne obtenue dans (i) est $\leq \operatorname{deg} p+1$, donc uniforme en $y \geq 1$. De même pour le nombre de zéros réels et pour la constante $d_{H, \frac{1}{2}}$.

Démonstration. Soit $h(y ; s)=p(s) \zeta^{*}(2 s) y^{s}$, alors

$$
H^{ \pm}(y ; s)=h(y ; s) \pm h(y ; 1-s)
$$

et on applique le théorème 4. Les zéros possibles de $h(y ; s)$ dans le demi-plan $\sigma \geq 1 / 2$ proviennent de $p(s)$, donc ils sont en nombre fini. Les estimations qui entraînent les conditions (ii) et (ii) du théorème 4 sont les mêmes que celles de la fonction $\zeta^{*}\left(s+\frac{1}{2}\right)$ étudiée au $\S 7$, sauf par le facteur dépendant du paramètre $y \geq 1$. Par la formule de Stirling, la fonction $F(y ; s)=h(y ; 1-s) / h(y ; s)$ satisfait, pour $\sigma \geq 1 / 2$ et $|s|$ assez grand

$$
\begin{aligned}
F(y ; s) & =y^{1-2 s} \frac{p(1-s)}{p(s)} \frac{\zeta^{*}(2 s-1)}{\zeta^{*}(2 s)} \\
& =y^{1-2 s} \frac{p(1-s)}{p(s)} \sqrt{\pi} \frac{\Gamma\left(s-\frac{1}{2}\right)}{\Gamma(s)} \frac{\zeta(2 s-1)}{\zeta(2 s)} \\
& =y^{1-2 s}(-1)^{\operatorname{deg} p}\left(1+O\left(|s|^{-1}\right)\right) \sqrt{\pi} s^{-1 / 2}\left(1+O\left(|s|^{-1}\right)\right) \frac{\zeta(2 s-1)}{\zeta(2 s)} .
\end{aligned}
$$

Donc

$$
F(y ; s)=y^{1-2 s}(-1)^{\operatorname{deg} p} \sqrt{\pi} s^{-1 / 2}\left(1+O\left(|s|^{-1}\right)\right) \frac{\zeta(2 s-1)}{\zeta(2 s)}
$$

pour $\sigma \geq \frac{1}{2}$ et $|s|$ assez grand. Pour $\sigma \rightarrow+\infty$, cette expression est $O\left(\sigma^{-1 / 2}\right)$. Pour $|\tau| \rightarrow+\infty$, l'expression est $O\left(|\tau|^{K}\right)$ pour une constante $K>0$. Donc, la fonction $F(y ; \cdot)$ satisfait les conditions du théorème 4, et on obtient (i) en remplaçant $P_{H, \sigma>\frac{1}{2}}=\chi_{H, 0}, N_{H, \sigma>\frac{1}{2}}=N_{p, \sigma>\frac{1}{2}}$ dans (5). La partie ((ii) du théorème est une conséquence immédiate de (i).

Il nous reste à montrer (iii). Pour obtenir (a) on utilise le lemme 7. De

$$
h(y ; s)=(2 s-1) q(s) \zeta^{*}(2 s) y^{s}=\frac{q(s)}{s} \xi(2 s) y^{s},
$$

on a

$$
\frac{\frac{\partial h}{\partial s}\left(y ; \frac{1}{2}\right)}{h\left(y ; \frac{1}{2}\right)}=\frac{q^{\prime}\left(\frac{1}{2}\right)}{q\left(\frac{1}{2}\right)}-2+2 \frac{\xi^{\prime}(1)}{\xi(1)}+\log y .
$$

On utilise maintenant la formule

$$
\frac{\xi^{\prime}(0)}{\xi(0)}=-\frac{\xi^{\prime}(1)}{\xi(1)}=-\frac{1}{2} \gamma-1+\frac{1}{2} \log 4 \pi
$$

[3, §11, p. 83] pour obtenir

$$
\frac{\frac{\partial h}{\partial s}\left(y ; \frac{1}{2}\right)}{h\left(y ; \frac{1}{2}\right)}=\frac{q^{\prime}\left(\frac{1}{2}\right)}{q\left(\frac{1}{2}\right)}+\gamma-\log 4 \pi+\log y=\log y-\log y^{*},
$$

donc la condition $y<y^{*}$ est équivalente à $\frac{\frac{\partial h}{\partial s}\left(y ; \frac{1}{2}\right)}{h\left(y ; \frac{1}{2}\right)}<0$, d'où on obtient (a). On sait que $\frac{\partial H^{-}}{\partial s}\left(y ; \frac{1}{2}\right)=$ $2 \frac{\partial h}{\partial s}\left(y ; \frac{1}{2}\right)$. Si $y=y^{*}$, alors $\frac{\partial H^{-}}{\partial s}\left(y ; \frac{1}{2}\right)=0$, puis $n_{H, \frac{1}{2}} \geq 2$ et puisque ce nombre est impair, on a $n_{H, \frac{1}{2}} \geq 3$, donc (b). Si $y>y^{*}$ on ne peut rien dire dans le cas général.

Sans perte de généralité, on peut prendre $q\left(\frac{1}{2}\right)>0$. Alors

$$
h\left(y ; \frac{1}{2}\right)=\lim _{s \rightarrow \frac{1}{2}}(2 s-1) \zeta^{*}(2 s) q(s) y^{s}=q(1 / 2) y^{1 / 2}>0 .
$$


L'analyse antérieure entraîne que $\frac{\partial H^{-}}{\partial s}\left(y ; \frac{1}{2}\right)=2 \frac{\partial h}{\partial s}\left(y ; \frac{1}{2}\right)>0$ si $y>y^{*}$. Donc, il existe $x_{0}>\frac{1}{2}$ tel que $H^{-}\left(y ; x_{0}\right)>0$. Maintenant, la condition $q(0)>0$ nous dit que $s=0$ n'est pas racine de $p(s)$, donc $H^{-}(y ; s)$ a un pôle en $s=1$, avec résidu $-q(0)<0$, d'où $\lim _{y \rightarrow 1^{-}} H^{-}(y ; s)=-\infty$. Donc, il existe $\rho \in] x_{0}, 1\left[\right.$ tel que $H^{-}(y ; \rho)=0$, ce qui montre $(\boldsymbol{c})$.

Maintenant on applique le théorème 18 pour obtenir comme des cas particuliers les résultats de $[20]$.

Corollaire 19 (Suzuki et Lagarias [20, Theorem 2]). Pour chaque $T \geq 1$ fixé, la fonction

$$
Z_{2, \mathbb{Q}}^{T}(s)=\zeta^{*}(2 s) \frac{T^{s-1}}{s-1}-\zeta^{*}(2-2 s) \frac{T^{-s}}{s}
$$

a tous ses zéros simples, et sur la droite $\sigma=\frac{1}{2}$.

Démonstration. Dans le théorème 18, on pose $p(s)=(2 s-1) s$. Alors

$$
H^{-}(T ; s)=T(2 s-1) s(s-1) I(T ; s),
$$

d'où l'on conclut par la partie (ii).

En particulier, pour $T=1$ on obtient l'hypothèse de Riemann pour $Z_{2, \mathbb{Q}}(s)$.

Corollaire 20 (Suzuki et Lagarias [20, Theorem 1]). La fonction zêta non-abélienne de rang $2 \operatorname{sur} \mathbb{Q}$

$$
Z_{2, \mathbb{Q}}(s)=\zeta^{*}(2 s) \frac{1}{1-s}-\zeta^{*}(2-2 s) \frac{1}{s}
$$

a tous ses zéros simples, et sur la droite $\sigma=\frac{1}{2}$.

Récemment, Hayashi [10] a obtenu une formule close pour la fonction zêta de Weng de rang 2 d'un corps de nombres quelconque [10], la même formule que pour le corps des nombres rationnels où on change la fonction zêta de Riemann complétée par la fonction de Dedekind complétée et à une constante près. À partir la formule obtenue, il obtient l'hypothèse de Riemann pour les fonctions de rang 2 par la méthode de Lagarias de Suzuki, la variante réelle du théorème 40.

L'hypothèse de Riemann pour la fonction zêta de rang 3 a été établie par Suzuki en [30]; on peut aussi appliquer nos méthodes à l'étude de cette fonction. La fonction zêta de rang 1 n'est autre que la fonction zêta complétée $\zeta^{*}(s)$.

On obtient maintenant une version modifiée de [20, Theorem 3] :

Corollaire 21. Soit $y^{*}=4 \pi e^{-\gamma}$. Pour chaque $y \geq 1$, la fonction

$$
a_{0}(y ; s)=\zeta^{*}(2 s) y^{s}+\zeta^{*}(2-2 s) y^{1-s}
$$

est une fonction méromorphe, qui peut être prolongée analytiquement au point $s=\frac{1}{2}$. Alors

a) Si $y<y^{*}$, tous les zéros de $a_{0}(y ; s)$ sont sur la droite critique $\sigma=\frac{1}{2}$ et ils sont simples.

b) Si $y=y^{*}$, tous les zéros de $a_{0}(y ; s)$ sont sur la droite critique $\sigma=\frac{1}{2}$ et ils sont simples, sauf $s=\frac{1}{2}$ qui est un zéro double.

c) Si $y>y^{*}$, il y a exactement deux zéros hors la droite critique, un zéro réel simple $\rho_{y}$ dans l'intervalle $] \frac{1}{2}, 1\left[\right.$, et son symétrique $1-\rho_{y}$. Tous les autres zéros sont sur la droite critique et ils sont simples.

Démonstration. On prend $p(s)=2 s-1$, d'où

$$
H^{-}(y ; s)=(2 s-1) a_{0}(y ; s) .
$$


Comme $p(0) \neq 0$, on ne peut pas appliquer la partie (ii) du théorème 18. Par la partie (ii) du même théorème, pour la fonction $H^{-}(y ; s)$ on a

$$
\begin{aligned}
N(T)-N_{0}(T) & \leq N(T)-N_{0}^{\prime}(T) \leq 2-N_{H, \sigma>\frac{1}{2}}-\frac{n_{H, \frac{1}{2}}}{2}-d_{H, \frac{1}{2}}, \\
& \leq \frac{3}{2}-n_{H, \sigma>\frac{1}{2}}-d_{H, \frac{1}{2}},
\end{aligned}
$$

puisque $n_{H, \frac{1}{2}} \geq 1$. On applique maintenant la partie (iii) du théorème. On a $q(s)=1$, donc $y^{*}=4 \pi e^{-\gamma}$ et

a) Si $y<y^{*}$, on a $d_{H, \frac{1}{2}} \geq 1$, puis forcément $n_{H, \sigma>\frac{1}{2}}=0$ et $n_{H, \frac{1}{2}}=1$.

b) Si $y=y^{*}$, on a $n_{H, \frac{1}{2}} \geq 3$, puis forcément $n_{H, \frac{1}{2}}=3$ et $n_{H, \sigma>\frac{1}{2}}=0$.

c) Si $y>y^{*}$, on a $n_{H, \sigma>\frac{1}{2}} \geq 1$, donc encore $n_{H, \sigma>\frac{1}{2}}=1$ et $n_{H, \frac{1}{2}}=1$.

Dans tous les cas, $N(T)=N_{0}(T)=N_{0}^{\prime}(T)$. Cela finit la preuve.

Remarques. Le résultat [20, Theorem 3] établit la continuité de $\rho_{y}$ en fonction du paramètre $y \geq 1$, en montrant que c'est une fonction croissante de $y$, et que $\rho_{y} \rightarrow 1$ lorsque $y \rightarrow+\infty$. L'alignement des zéros non-réels de $a_{0}(y ; s)$ a été demontré par Hejhal en [11, Proposition 5.3 (f)] en utilisant les relations de Maaß-Selberg [34, §2.18]. La simplicité des zéros de $a_{0}(y, s)$ a été récemment montrée par $\mathrm{H}$. Ki en [18]. On remarque que ces approches traitent l'alignement et la simplicité des zéros séparément.

Récemment, W. Müeller a donné en [23] une interprétation des zéros de $a_{0}(y ; s)$ en termes des valeurs propres d'un opeŕateur autoadjoint, un pseudo-laplacien agissant sur un sous-espace de $L^{2}(\operatorname{PSL}(2, \mathbb{Z}) \backslash \mathbb{H})$. Dans ce travail, il fournit aussi une extension du corollaire 21 au terme constant d'une série d'Eisenstein associée à $\operatorname{PSL}\left(2, \mathcal{O}_{\mathbb{K}}\right)$ [23, Theorem 0.3$]$, où $\mathcal{O}_{\mathbb{K}}$ est l'anneau des entiers d'un corps quadratique imaginaire $\mathbb{K}=\mathbb{Q}(\sqrt{-d}), d>0$ entier, tel que le nombre de classes d'idéaux entiers $h(-d)=1$. Ce terme est obtenue en remplaçant la fonction zêta de Riemann complétée par la fonction zêta de Dedekind complétée dans (23); nos méthodes s'étendent facilement à ce cadre.

Quant à la répartition des zéros, une application directe du théorème 6 et la remarque faite au théorème 18 nous fournissent le résultat suivant.

Théorème 22. Pour chaque $y \geq 1$, la fonction $H^{ \pm}(y ; s)$ dans le théorème 18 a tous ses zéros, sauf un nombre fini, simples et alignés sur la droite $\sigma=\frac{1}{2}$. Le nombre de ces zéros, en fonction du paramêtre $y \geq 1$, avec $0<\tau<T$ est

$$
N(y ; T)=\frac{T}{\pi} \log T-\frac{1}{\pi}(\log \pi+1) T+\frac{1}{\pi}(\log y) T+O(\log T) .
$$

La constante dans $O(\cdot)$ ne dépend pas de $y \geq 1$. Les zéros des fonctions $H^{ \pm}(y ; s)$ sont ultimement entrelacés.

Cette estimation est la même que celle calculée par Lagarias et Suzuki [20, Theorem 5] pour la fonction $Z_{2, \mathbb{Q}}^{T}(s)$. Ils montrent de plus que la partie imaginaire de chaque zéro de $Z_{2, \mathbb{Q}}^{T}(s)$ avec $\tau>0$ est une fonction strictement décroissante de $T$.

Il nous reste à voir le cas où $0<y<1$. De (25), la fonction $F(y ; s)=\frac{h(y ; 1-s)}{h(y ; s)}$ satisfait, pour $\sigma$ suffisament grand, $F(y ; s)=O\left(y^{-2 \sigma}|s|^{-1 / 2}\right)$. Puisqu'elle contient une puissance de $y$ convergeant vers l'infini lorsque $\sigma \rightarrow+\infty$, cette fonction ne satisfait pas les conditions du thèorème 4 .

La proposition suivante a été énoncée par D. Hehjal en [11, p. 89], et montrée par H. Ki [16, $\S 2]$ dans le cas où $p(s)=1$.

Proposition 23. Pour $0<y<1$, la fonction $H^{ \pm}(y, s)$ du théorème 18 possède des zéros avec parties réelles arbitrairement grandes. Pour $\sigma_{0}$ suffisamment grand, le nombre de zéros de $H(y, s)$ avec $\sigma \geq \sigma_{0}$ et $0<\tau<T$ satisfait

$$
N_{H}\left(\sigma_{0}, T\right)=O(T) \text {. }
$$


Démonstration. Les zéros de $H(y ; s)$ sont, essentiellement (sauf un nombre fini), ceux de la fonction $g(s)=1 \pm F(y ; s)$. Par l'équation (25), $g(s)=1 \pm y^{1-2 s} \sqrt{\pi} s^{-1 / 2}\left(1+O\left(\sigma^{-1}\right)\right)$ pour $\sigma \geq \sigma_{0}$. Cette fonction a le même comportement que la fonction

$$
g_{1}(s)=1 \pm y^{1-2 s} \sqrt{\pi} s^{-1 / 2},
$$

comparable à une exponentielle-polynôme sans terme principal [1, Chapter 13]. Le principe de l'argument appliqué à $g_{1}(s)$ montre que son nombre de zéros avec $0<\tau<T$ est $c T+O(1)$ pour $c=-\frac{1}{\pi} \log y>0$ [25, Part III, Chapter 4, Problem 206.2]. Le théorème de Rouché transmet cette information à $g(s)$ dans le demi-plan $\sigma \geq \sigma_{0}$.

Or, chaque racine de $g(s)$ vérifie $|s|^{1 / 2}=y^{1-2 \sigma} \sqrt{\pi}\left(1+O\left(\sigma^{-1}\right)\right)$. Pour une suite de zéros de $g(s)$ (vu qu'il y a une infinité), $|s| \rightarrow+\infty$, d'où de cette dernière égalité $\sigma \rightarrow+\infty$.

On parlera ensuite des autres zéros de $H(y, s)$, ceux dans la bande $|\sigma-a|<\sigma_{0}$.

\section{Dominance faible. Un résultat non effectif.}

La nécéssité d'étendre le thèorème 18 au cas $0<y<1$, qui n'est pas de la même nature que le cas $y \geq 1$, nous motive à établir le résultat suivant.

Théorème 24. Soit $\sigma_{0}>a, h(s)$ une fonction méromorphe, réelle sur la droite réelle, avec un nombre fini de pôles, un nombre fini de zéros dans la bande $a<\sigma<\sigma_{0}$; holomorphe et non nulle sur la droite $\sigma=\sigma_{0}$. On pose

$$
f(s)=f^{ \pm}(s)=h(s) \pm h(2 a-s) .
$$

On suppose que la fonction

$$
F(s)=\frac{h(2 a-s)}{h(s)}
$$

satisfait

(i”) $F(s) \neq \pm 1$ sur $\sigma=\sigma_{0}$, et il existe $\tau_{0}>0$ tel que $|F(s)|<1$ pour $\sigma=\sigma_{0},|\tau| \geq \tau_{0}$;

(ii") il existent $K>0$ et une suite $\left(T_{n}\right)_{n}$ telle que $\lim _{n \rightarrow \infty} T_{n}=+\infty$ et $|F(s)|<e^{K|s|}$ pour $a \leq \sigma \leq$ $\sigma_{0},|\tau|=T_{n}, n \geq 1$.

On note le nombre des zéros de $f(s)$ avec $0<\tau<T$ dans la bande $|\sigma-a|<\sigma_{0}-a$ (en comptant les multiplicités) par

$$
N\left(T, \sigma_{0}\right)=\#\left\{s \in \mathbb{C}|| \sigma-a \mid<\sigma_{0}-a, 0<\tau<T\right\} .
$$

Alors, il existe une constante $B=B\left(\sigma_{0}\right)$ telle que

$$
N\left(T, \sigma_{0}\right)-N_{0}^{\prime}(T) \leq B
$$

pour $T>0$. Donc tous les zéros de $f(s)$ dans la bande $|\sigma-a|<\sigma_{0}-a$, sauf un nombre fini, sont sur la droite $\sigma=a$ et ils sont simples.

Remarque. En comparant avec les conditions du théorème 4, la condition (i”) est l'analogue de (i) avec $\eta=1$, alors que (ii") est l'analogue de (iii) avec un $K>0$ fixé au lieu d'un $\varepsilon>0$ arbitraire.

Démonstration. On suit la démarche de la démonstration du théorème 4. D'après la remarque (a) à ce théorème, on peut supposer que $F(s)$ est regulière et non nulle sur la droite $\sigma=a$.

On considère le rectangle $R$ défini par

$$
-T \leq \tau \leq T, \quad 2 a-\sigma_{0} \leq \sigma \leq \sigma_{0},
$$


tel que $f(s)$ n'a pas ni de zéro ni de pôle sur sa frontière. On calcule $N\left(T, \sigma_{0}\right)$ comme on le fait pour $N(T)$ dans le théorème 4, en calculant la variation d'argument des fonctions $g(s)$ et $h(s)$ (avec $f(s)=h(s) g(s)$ ) le long du chemin donné par les deux segments qui lient dans l'ordre $\sigma_{0}$, $\sigma+i T, s$

$$
R(T)=\frac{1}{\pi} \Delta \arg h(s), \quad S(T)=\frac{1}{\pi} \Delta \arg g(s) .
$$

On remarque que ces deux quantités dépendent de $\sigma_{0}$ en général, dans ce cas fixe. L'application du lemme de Littlewood nous fournit la formule

$$
\pi \int_{0}^{T_{n}} S(\tau) d \tau=\int_{a}^{\sigma_{0}} \log \left|g\left(\sigma+i T_{n}\right)\right| d \sigma+\int_{0}^{T_{n}} \arg g\left(\sigma_{0}+i \tau\right) d \tau-I\left(\sigma_{0}\right) .
$$

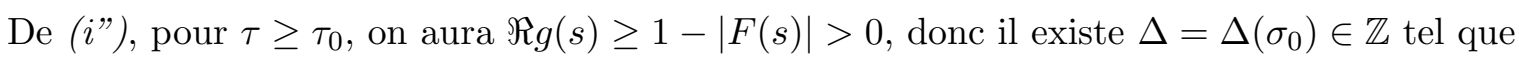

$$
\left|\arg g\left(\sigma_{0}+i \tau\right)-2 \pi \Delta\right|<\pi
$$

pour $\tau \geq \tau_{0}$. Puis

$$
\pi \int_{0}^{T_{n}} S(\tau) d \tau \leq \sigma_{0} K\left(\sigma_{0}+T_{n}\right)+\int_{0}^{\tau_{0}} \arg g\left(\sigma_{0}+i \tau\right) d \tau+\left(T_{n}-\tau_{0}\right)(2 \Delta+1) \pi-I\left(\sigma_{0}\right)
$$

au lieu de (8). La contribution correspondante de la moyenne de $S(T)$, obtenue en divisant cette dernière inégalité par $\pi T_{n}$ et en faisant $n \rightarrow+\infty$, est $\leq \sigma_{0} K+2 \Delta+1$, donc finie. La quantité analogue de $B_{a}$ est obtenue en calculant le bilan entre le nombre s zéros et les pôles de $f(s)$ et $h(s)$ avec $\sigma<\sigma_{0}$, donc qu'on note par $B_{a}\left(\sigma_{0}\right)$. L'argument de démonstration du théorème 4 nous donne finalement

$$
N\left(\sigma_{0}, T\right)-N_{0}^{\prime}(T) \leq B_{a}\left(\sigma_{0}\right)+\sigma_{0} K+2 \Delta+1,
$$

ce qui montre le résultat.

Le point faible du théorème 24 est l'innefectivité de la borne 26, qui dépend de la quantité $\Delta$, difficile à calculer; c'est la raison pour laquelle on ne garde pas la borne obtenue dans l'énoncé du théorème.

On peut maintenant compléter l'information du théorème 18 et la proposition 23 dans le cas $0<y<1$.

Proposition 25. Soit $0<y<1, H(y ; s)$ comme dans le théorème 18. Alors, pour tout $\sigma_{0}>1 / 2$, tous les zéros de $H(y ; s)$ dans la bande $|\sigma-1 / 2|<\sigma_{0}-1 / 2$, sauf un nombre fini, sont sur la droite $\sigma=1 / 2$ et ils sont simples.

Démonstration. Il suffit de vérifier la condition $(i 》)$ du thèorème 24 pour $\sigma=\sigma_{0}$ suffisament grand. On pose $F(y ; s)=\frac{h(y ; 1-s)}{h(y ; s)}$. Par (25), pour $\sigma$ suffisament grand, $F(y ; s)=O\left(y^{-2 \sigma}|s|^{-1 / 2}\right)$. Donc, pour $\sigma_{0}$ assez grand, $|F(s)|=O_{\sigma_{0}}\left(|\tau|^{-1 / 2}\right)$ pour $\sigma=\sigma_{0},|\tau| \geq \tau_{0}$.

D'un autre côté, pour $\sigma_{0}$ fixé, la puissance $y^{-2 \sigma}$ est bornée pour $a \leq \sigma \leq \sigma_{0}$, ce qui suffit pour vérifier (ii”) avec (25) et les arguments habituels. Le résultat se suit directement.

Le dernier résultat n'est pas nouveau, il a été obtenu par H. Ki, il correspond à un cas particulier de la proposition 29. Notre méthode de démonstration diffère de celle de H. Ki dans le sens qu'on n'a pas d'analyse de derivée à faire, pour un résultat sur la simplicité des zéros etudiés. La généralité du théorème 24 nous permettra de l'appliquer pour étudier la répartition des zéros des approximations de la fonction zêta d'Epstein, au §12. 


\section{Approximations de la fonction zêta d'Epstein}

Soit $Q(u, v)=a u^{2}+b u v+c v^{2}$ avec $a>0, b, c \in \mathbb{R}$ une forme quadratique définie positive, c'est-à-dire avec $\Delta=4 a c-b^{2}>0$. La fonction zêta d'Epstein associée à $Q(u, v)$ est donnée par

$$
Z_{Q}(s)=\sum_{(m, n) \neq(0,0)} Q(m, n)^{-s}
$$

pour $\sigma>1$. Cette fonction peut être prolongée analytiquement dans le plan complexe, sauf en $s=1$, où elle a un pôle simple, et elle satisfait à l'équation fonctionnelle

$$
\left(\frac{\sqrt{\Delta}}{2 \pi}\right)^{s} \Gamma(s) Z_{Q}(s)=\left(\frac{\sqrt{\Delta}}{2 \pi}\right)^{1-s} \Gamma(1-s) Z_{Q}(1-s) .
$$

Cette fonction est essentiellement une série d'Eisenstein; on change notre point de vue pour des raisons historiques. Lorsque les coefficients $a, b, c$ sont des entiers, qu'on suppose avec $(a, b, c)=1$, l'étude de cette fonction est liée à l'étude du corps quadratique imaginaire $\mathbb{Q}(\sqrt{-d})$, où $d=\Delta / 4$ si $\Delta / 4$ est entier, $d=\Delta$ sinon. Soit $h(-d)$ le nombre de classes d'idéaux entiers de $\mathbb{Q}(\sqrt{-d})$ (ou des classes d'équivalence de formes quadratiques de discriminant $-\Delta$ ). Si $h(-d)=1$, la fonction zêta d'Epstein est, à une constante près, la fonction zêta de Dedekind du corps quadratique $\mathbb{Q}(\sqrt{-d})$, elle a un produit eulérien, et contient la fonction zêta de Riemann comme facteur (on reprend l'exemple de la série d'Eisenstein $E(i, s)$, le cas où $\left.Q(u, v)=u^{2}+v^{2}\right)$. Dans ce cas, on attend que tous les zéros de $Z_{Q}(s)$ soient sur la droite critique $\sigma=1 / 2$, une hypothèse de Riemann pour $Z_{Q}(s)$. Par contre, celui-ci est le cas moins fréquent, puisque les seuls entiers positifs $d$ pour lesquels $h(-d)=1$ sont les nombres de Heegner $d=3,4,7,8,11,19,43,67,163$. Si $h(-d)>1$, la fonction zêta d'Epstein n'a pas de produit eulérien, et elle a une infinité de zéros en dehors de la droite $\sigma=1 / 2$. Plus précisement, il a été montré par Davenport et Heilbronn dans [4] et [5], que, lorsque $h(-d)>1$, la fonction $Z_{Q}(s)$ a une infinité de zéros dans le demi-plan $\sigma>1$, arbitrairement proches de la droite $\sigma=1$, et par Voronin dans [35] que dans une bande $\sigma_{1} \leq \sigma \leq \sigma_{2}$ avec $1 / 2<\sigma_{1}<\sigma_{2}<1$, cette fonction a au moins $c\left(\sigma_{1}, \sigma_{2}\right) T$ zéros avec $0<\tau<T$ pour $T$ suffisament grand, où $c\left(\sigma_{1}, \sigma_{2}\right)>0$. Bien sûr, cette fonction a une infinité de zéros sur la droite $\sigma=1 / 2$, ce qui a été montré par Potter et Titchmarsh [27], et le nombre des zéros avec $0<\tau<T$ est asymptotiquement $c T \log T$ (une formule explicite bien sûr, analogue à celle pour la fonction zêta de Riemann).

On reprend la formule de Chowla-Selberg

$$
\begin{aligned}
Z_{Q}(s)= & 2 \zeta(2 s) a^{-s}+\frac{2^{2 s} a^{s-1} \sqrt{\pi}}{\Gamma(s) \Delta^{s-1 / 2}} \zeta(2 s-1) \Gamma\left(s-\frac{1}{2}\right) \\
& +\frac{4 \pi^{s} 2^{s-1 / 2}}{\sqrt{a} \Gamma(s) \Delta^{s / 2-1 / 4}} \sum_{n=1}^{\infty} n^{s-1 / 2} \sum_{d \mid n} d^{1-2 s} \cdot \cos \frac{n \pi b}{a} \int_{-\infty}^{+\infty} e^{-(\pi n \sqrt{\Delta} / a) \cosh \tau} e^{(s-1 / 2) \tau} d \tau
\end{aligned}
$$

et on obtient des approximations de la fonction zêta d'Epstein en prenant les sommes partielles des termes dans cette formule. Pour $n \geq 1$ entier, on définit $Z_{Q, n}(s)$ par

$$
\begin{aligned}
Z_{Q, n}(s)= & 2 \zeta(2 s) a^{-s}+\frac{2^{2 s} a^{s-1} \sqrt{\pi}}{\Gamma(s) \Delta^{s-1 / 2}} \zeta(2 s-1) \Gamma\left(s-\frac{1}{2}\right) \\
& +\frac{4 \pi^{s} 2^{s-1 / 2}}{\sqrt{a} \Gamma(s) \Delta^{s / 2-1 / 4}} \sum_{k=1}^{n} k^{s-1 / 2} \sum_{d \mid k} d^{1-2 s} \cdot \cos \frac{k \pi b}{a} \int_{-\infty}^{+\infty} e^{-(\pi k \sqrt{\Delta} / a) \cosh \tau} e^{(s-1 / 2) \tau} d \tau .
\end{aligned}
$$

Ces fonctions ont été précédemment étudiées par D. Hehjal dans [11, §5], et reprises par H. Ki dans [16], qui améliore ses résultats. On va redémontrer les résultats de $\mathrm{H}$. Ki avec nos techniques. 
On définit pour cela une classe de fonctions qui généralise $Z_{Q, n}(s)$. Soient $\lambda>0, A_{j}>0, \lambda_{j}>0$ et $b_{j} \in \mathbb{R}$ pour $j=1, \ldots, n$. On note par $G(s)$ la fonction

$$
\begin{aligned}
G(s)= & \zeta(2 s) \lambda^{s}+\sqrt{\pi} \frac{\Gamma\left(s-\frac{1}{2}\right)}{\Gamma(s)} \zeta(2 s-1) \lambda^{1-s} \\
& +\frac{\pi^{s}}{\Gamma(s)} \sum_{k=1}^{n} b_{k}\left(\lambda_{k}^{s-1 / 2}+\lambda_{k}^{1 / 2-s}\right) \int_{-\infty}^{+\infty} e^{-2 A_{k} \cosh \tau} e^{(s-1 / 2) \tau} d \tau .
\end{aligned}
$$

On voit facilement que $\frac{1}{2}(\sqrt{\Delta} / 2)^{s} Z_{Q, n}(s)$ est un cas particulier de $G(s)$, où $\lambda=\sqrt{\Delta} /(2 a)$ et sous les conditions aditionnelles $0<\lambda_{j} \leq 1$ et $\lambda_{j} A_{j} \geq \pi \lambda$ pour $j=1, \ldots, n$.

La parité de la fonction de Bessel et l'équation fonctionnelle de la fonction zêta de Riemann entraînent l'équation fonctionnelle pour $G(s)$

$$
\pi^{-s} \Gamma(s) G(s)=\pi^{-1+s} \Gamma(1-s) G(1-s),
$$

qui à son tour, entraîne celle de la fonction $Z_{Q, n}(s)$

$$
\left(\frac{\sqrt{\Delta}}{2 \pi}\right)^{s} \Gamma(s) Z_{Q, n}(s)=\left(\frac{\sqrt{\Delta}}{2 \pi}\right)^{1-s} \Gamma(1-s) Z_{Q, n}(1-s)
$$

En fait, l'équation fonctionnelle de $G(s)$ est une conséquence directe de l'egalité

$$
\pi^{-s} \Gamma(s) G(s)=w(s)+w(1-s),
$$

où

$$
w(s)=\lambda^{s} \pi^{-s} \Gamma(s) \zeta(2 s)+\sum_{k=1}^{n} b_{k} \lambda_{k}^{s-\frac{1}{2}} 2 K_{s-\frac{1}{2}}\left(2 A_{k}\right) .
$$

L'estimation suivante est analogue à celle dans [16, Proposition 3.5].

Proposition 26. Il existe $R>0$ tel que

$$
\frac{K_{s-1 / 2}(2 A)}{\Gamma(s)}=O\left(\frac{A^{-\sigma}}{|s|^{1 / 2}}\right)
$$

pour $|s|>R, \sigma \geq \frac{1}{2}$.

Démonstration. La formule dans [24, p. 309]

$$
\begin{aligned}
K_{s}(2 A)= & A^{-s} \Gamma(s)\left(1+\sum_{n=1}^{\infty} \frac{A^{2 n}}{n !(1-s)(2-s) \cdots(n-s)}\right) \\
& +\Gamma(-s)\left(1+\sum_{n=1}^{\infty} \frac{A^{2 n}}{n !(1+s)(2+s) \cdots(n+s)}\right)
\end{aligned}
$$

permet de lier les comportements asymptotiques de la fonction de Bessel et de la fonction gamma. Il a été montré par Pólya dans [24, pp. 309-310] que la fonction entière $\psi_{A}(s)=A^{s} K_{s}(2 A) / \Gamma(s)-1$ satisfait $\lim _{|s| \rightarrow \infty} \psi_{a}(s)=0$ dans tout demi-plan $\sigma \geq \varepsilon, \varepsilon>0$ fixé. Le même calcul montre que $\psi_{a}(s)$ est bornée dans le demi-plan $\sigma \geq 0$. Or

$$
\frac{K_{s-1 / 2}(2 A)}{\Gamma(s)}=\left(1+\psi_{a}(s-1 / 2)\right) A^{1 / 2-s} \frac{\Gamma(s-1 / 2)}{\Gamma(s)} .
$$

On applique la formule de Stirling pour obtenir le résultat.

Ensuite on énonce et démontre une variante du résultat de H. Ki [16, Theorem 1]. 
Proposition 27. Soient $\lambda>0, A_{j}>0, \lambda_{j}>0$ et $b_{j} \in \mathbb{R}$ pour $j=1, \ldots, n$, et $G(s)$ la fonction définie par (27). Alors, pour tout $\sigma_{0}>1 / 2$, tous les zéros de $G(s)$ dans la bande $|\sigma-a|<\sigma_{0}-a$, sauf un nombre fini, sont simples et sur la droite $\sigma=\frac{1}{2}$.

Sous les conditions additionnelles $\lambda \geq 1,0<\lambda_{j} \leq 1$ et $\lambda_{j} A_{j} \geq \pi \lambda$ pour $j=1, \ldots, n$, tous les zéros non triviaux de $G(s)$, sauf un nombre fini, sont simples et sur la droite $\sigma=\frac{1}{2}$.

Corollaire 28 (H. Ki [16, Corollary 1]). Pour les fonctions $Z_{Q, n}(s)$, on a le résultat de la proposition 27. Si $\sqrt{\Delta} /(2 a) \geq 1$, tous les zéros non triviaux de $Z_{Q, n}(s)$, sauf un nombre fini, sont simples et sur la droite $\sigma=\frac{1}{2}$.

Démonstration (de la proposition). Les zéros triviaux des fonctions $G(s)$ et $Z_{Q, n}(s)$ proviennent de la fonction gamma, comme pour la fonction zêta de Riemann. On va appliquer les théorèmes 4 et 24 . Il est clair que les seuls pôles de $w(s)$ sont $s=0, s=1 / 2$. On pose

$$
\mu=\max _{1 \leq k \leq n} \lambda_{k}^{-1} A_{k}^{-1} \pi \lambda^{-1}, \quad \nu=\max _{1 \leq k \leq n} \lambda_{k} A_{k}^{-1} \pi \lambda^{-1} .
$$

On factorise $w(s)$ par

$$
w(s)=\left(\frac{\lambda}{\pi}\right)^{s} \Gamma(s)\left(\zeta(2 s)+\left(\frac{\lambda}{\pi}\right)^{-s} \sum_{k=1}^{n} 2 b_{k} \lambda_{k}^{s-\frac{1}{2}} \frac{K_{s-\frac{1}{2}}\left(2 A_{k}\right)}{\Gamma(s)}\right) .
$$

D'après la proposition 26 , pour $|s|$ suffisament grand, $\sigma \geq 1 / 2,1 \leq k \leq n$

$$
\left(\frac{\lambda}{\pi}\right)^{-s} \lambda_{k}^{s-\frac{1}{2}} \frac{K_{s-\frac{1}{2}}\left(2 A_{k}\right)}{\Gamma(s)}=O\left(\lambda^{-\sigma} \pi^{\sigma} \lambda_{k}^{\sigma} \frac{A_{k}^{-\sigma}}{|s|^{1 / 2}}\right)=O\left(\frac{\nu^{\sigma}}{|s|^{1 / 2}}\right)
$$

d'où

$$
w(s)=\left(\frac{\lambda}{\pi}\right)^{s} \Gamma(s)\left(\zeta(2 s)+O\left(\nu^{\sigma}|s|^{-1 / 2}\right)\right)
$$

et $\zeta(2 s)^{-1}=O\left(|s|^{1 / 4}\right)$ pour $\sigma \geq 1 / 2$, puis

$$
w(s)=\left(\frac{\lambda}{\pi}\right)^{s} \Gamma(s) \zeta(2 s)\left(1+O\left(\nu^{\sigma}|s|^{-1 / 4}\right)\right),
$$

pour $\sigma \geq 1 / 2$ et $|s|$ suffisament grand.

Si l'on écrit $F(s)=w(1-s) / w(s)$,

$$
\begin{aligned}
F(s)= & \frac{\lambda^{1-s} \pi^{\frac{1}{2}-s} \Gamma\left(s-\frac{1}{2}\right) \zeta(2 s-1)+\sum_{k=1}^{n} b_{k} \lambda_{k}^{\frac{1}{2}-s} 2 K_{s-\frac{1}{2}}\left(2 A_{k}\right)}{\left(\frac{\lambda}{\pi}\right)^{s} \Gamma(s) \zeta(2 s)\left(1+O\left(\nu^{\sigma} \sigma^{-1 / 4}\right)\right)} \\
= & \frac{\lambda^{1-2 s} \sqrt{\pi} \frac{\Gamma\left(s-\frac{1}{2}\right)}{\Gamma(s)} \zeta(2 s-1)+\sum_{k=1}^{n} b_{k} \lambda_{k}^{\frac{1}{2}-s} \lambda^{-s} \pi^{s} 2 \frac{K_{s-\frac{1}{2}}\left(2 A_{k}\right)}{\Gamma(s)}}{\zeta(2 s)\left(1+O\left(\nu^{\sigma}|s|^{-1 / 4}\right)\right)} .
\end{aligned}
$$

Or, encore par la proposition 26 , on a pour $|s|$ assez grand, $\sigma \geq 1 / 2,1 \leq k \leq n$

$$
\lambda_{k}^{\frac{1}{2}-s} \lambda^{-s} \pi^{s} \frac{K_{s-\frac{1}{2}}\left(2 A_{k}\right)}{\Gamma(s)}=O\left(\lambda^{-\sigma} \pi^{\sigma} \lambda_{k}^{-\sigma} \frac{A_{k}^{-\sigma}}{|s|^{1 / 2}}\right)=O\left(\frac{\mu^{\sigma}}{|s|^{1 / 2}}\right) .
$$

Puis, pour $\sigma \geq 1 / 2,|s|$ suffisament grand

$$
F(s)=\frac{O\left(\lambda^{-2 \sigma}|s|^{-1 / 2}\right) \zeta(2 s-1)+O\left(\mu^{\sigma}|s|^{-1 / 2}\right)}{\zeta(2 s)\left(1+O\left(\nu^{\sigma}|s|^{-1 / 4}\right)\right)} .
$$


a) Soit $\sigma_{0}>1 / 2$ tel que $w(s) \neq 0$ et $F(s) \neq \pm 1$ sur $\sigma=\sigma_{0}$, et $\zeta(s) \asymp 1$ pour $\sigma \geq \sigma_{0}$ $\left(\sigma_{0}>2\right)$. Dans (31), on fait $|\tau| \rightarrow+\infty$ pour $s=\sigma_{0}+i \tau$, et on obtient $F(s)=O_{\sigma_{0}}\left(|\tau|^{-1 / 2}\right)$, d'où la condition ( $i$ ”) du théorème 24. Pour $1 / 2 \leq \sigma \leq \sigma_{0}$, les puissances $C^{\sigma}$ avec $C$ constante, agissent comme des constantes. Puis, les estimations $\zeta(2 s-1)=O\left(|\tau|^{A}\right)$ et $\zeta(2 s)^{-1}=O(\log |\tau|)$ pour $\sigma \geq 1 / 2$ et $|\tau|$ assez grand, entraînent que $F(s)=O_{\sigma_{0}}\left(|\tau|^{A} \log |\tau|\right)$ pour $1 / 2 \leq \sigma \leq \sigma_{0},|\tau| \geq \tau_{0}$, donc la condition (ii”) du théorème 24. La première partie de la proposition découle de ce résultat.

b) On suppose maintenant les conditions $\lambda \geq 1,0<\lambda_{j} \leq 1$ et $\lambda_{j} A_{j} \geq \pi \lambda$ pour $j=1, \ldots, n$. Alors, $\mu \leq 1$ et $\nu \leq 1$. La fonction $w(s)$ n'a qu'un nombre fini de zéros avec $\sigma \geq 1 / 2$, puisque dans (30) $w(s)=\lambda^{s} \zeta^{*}(2 s)\left(1+O\left(|s|^{-1 / 4}\right)\right)$, puis $w(s) \neq 0$ pour $|s|$ assez grand, $\sigma \geq 1 / 2$. L'équation (31) devient

$$
F(s)=\frac{O\left(|s|^{-1 / 2}\right) \zeta(2 s-1)+O\left(|s|^{-1 / 2}\right)}{\zeta(2 s)\left(1+O\left(|s|^{-1 / 4}\right)\right)}
$$

Lorsque $\sigma \rightarrow+\infty$, on a $F(s)=O\left(\sigma^{-1 / 2}\right)$ uniformément en $\tau$, donc la condition (i) du théorème 4. Lorsque $\tau \rightarrow+\infty$, on a $F(s)=O\left(|\tau|^{A} \log |\tau|\right)$ pour $\sigma \geq 1 / 2,|\tau|$ assez grand, donc la condition (iii) du théorème 4 (on fait on a dejà montré la condition souhaitée, mais on a mieux qu'en (a)). Le théorème 4 nous donne la seconde partie de la proposition, ainsi que le corollaire 28.

Dans le cas $Q(u, v)=u^{2}+v^{2}$, on a $\lambda=\sqrt{\Delta} /(2 a)=1$, d'où l'interêt de ce résultat. L'avantage du théorème 4 sur le théorème 24 est la majoration effective du nombre de zéros de $G(s)$ en dehors de la droite critique, le cas où on pourrait estimer le nombre de zéros de la fonction $w(s)$ définie par (28) dans le demi-plan $\sigma \geq 1 / 2$.

Dans le cas où $0<\lambda<1$, H. Ki calcule aussi le nombre de zéros des fonctions $G(s)$ hors une bande $|\sigma-a|<\sigma_{0}-a$ fixée et $0<\tau<T$, pour montrer qu'il est $O(T)$, en utilisant une approximation des fonctions de Bessel [16, Proposition 3.5], pour approcher $G(s)$ avec une fonction semblable à une exponentielle-polynôme dans le demi-plan $\sigma \geq \sigma_{0}$, comme dans la proposition 23 .

H. Ki a aussi étudié des fonctions zêta d'Epstein modifiées, dans [17]. On cite le texte original. Soient $L_{1}(s), \ldots, L_{n}(s)$ des séries de Dirichlet qui peuvent être continuées analytiquement au plan complexe, sauf en un nombre fini de points. On suppose que

(i) $y>0$;

(ii) $\alpha$ et $\beta$ sont des polynômes tels que $\operatorname{deg}(\alpha) \geq \operatorname{deg}(\beta)+1$ et $\beta(s)=\beta(1-s)$;

(iii) $a_{k}$ est réel pour $k=1, \ldots, n$;

(iv) $\pi^{-s} \Gamma(s) L_{k}(s)=\pi^{-1+s} \Gamma(1-s) L_{k}(1-s)$, pour $k=1,2, \ldots, n, s \in \mathbb{C} \backslash\{0,1\}$;

(v) il existe $\varepsilon>0$ tel que $L_{k}(s)=O\left(|s|^{1-\varepsilon}\right)$, pour $k=1,2, \ldots, n, \sigma \geq \frac{1}{2}$.

On définit $Z\left(s ; y, \alpha, \beta, L_{1}, \ldots, L_{n}\right)$ par

$Z\left(s ; y, \alpha, \beta, L_{1}, \ldots, L_{n}\right)=\alpha(s) \zeta(2 s)+\alpha(1-s) \sqrt{\pi} \frac{\Gamma(s-1 / 2)}{\Gamma(s)} \zeta(2 s-1) y^{1-2 s}+y^{-s} \beta(s) \sum_{k=1}^{n} a_{k} L_{k}(s)$.

En fait, si

$$
w(s)=\alpha(s) y^{s} \zeta^{*}(2 s)+\frac{\beta(s)}{2} \sum_{k=1}^{n} a_{k} \pi^{-s} \Gamma(s) L_{k}(s)
$$

on a

$$
\pi^{-s} \Gamma(s) y^{s} Z\left(s ; y, \alpha, \beta, L_{1}, \ldots, L_{n}\right)=w(s)+w(1-s) .
$$


Puis, on a l'équation fonctionnelle

$$
\left(\frac{y}{\pi}\right)^{s} \Gamma(s) Z\left(s ; y, \alpha, \beta, L_{1}, \ldots, L_{n}\right)=\left(\frac{y}{\pi}\right)^{1-s} \Gamma(1-s) Z\left(1-s ; y, \alpha, \beta, L_{1}, \ldots, L_{n}\right)
$$

Proposition 29 (H. Ki [17, Theorem 1.1]). Pour tout $\sigma_{0}>1 / 2$, tous les zéros non-triviaux de la fonction $Z\left(s ; y, \alpha, \beta, L_{1}, \ldots, L_{n}\right)$ dans la bande $|\sigma-1 / 2|<\sigma_{0}-1 / 2$, sauf un nombre fini, sont simples et sur la droite $\sigma=1 / 2$.

Corollaire 30 (H. Ki [17, Corollary 1.2]). Si $y \geq 1$, tous les zéros de $Z\left(s ; y, \alpha, \beta, L_{1}, \ldots, L_{n}\right)$, sauf un nombre fini, sont simples et sur la droite $\sigma=\frac{1}{2}$.

Démonstration. Par (ii) et (v), il existe $\varepsilon>0$ tel que

$$
\begin{aligned}
w(s) & =\alpha(s) y^{s} \pi^{-s} \Gamma(s)\left(\zeta(2 s)+y^{-s} \frac{\beta(s)}{2 \alpha(s)} \sum_{k=1}^{n} a_{k} L_{k}(s)\right) \\
& =\alpha(s) y^{s} \pi^{-s} \Gamma(s)\left(\zeta(2 s)+O\left(y^{-\sigma}|s|^{-\varepsilon}\right)\right)
\end{aligned}
$$

et $\zeta(2 s)=O\left(|s|^{\varepsilon / 2}\right)$ pour $\sigma \geq 1 / 2$, puis

$$
w(s)=\alpha(s) y^{s} \pi^{-s} \Gamma(s) \zeta(2 s)\left(1+O\left(y^{-\sigma}|s|^{-\varepsilon / 2}\right)\right)
$$

pour $\sigma \geq \frac{1}{2},|s|$ assez grand.

D'un autre côté, $F(s)=w(1-s) / w(s)$ satisfait

$$
\begin{aligned}
F(s) & =\frac{\alpha(1-s) y^{1-s} \pi^{\frac{1}{2}-s} \Gamma\left(s-\frac{1}{2}\right) \zeta(2 s-1)+\frac{\beta(s)}{2} \sum_{k=1}^{n} a_{k} \pi^{-s} \Gamma(s) L_{k}(s)}{\alpha(s) y^{s} \pi^{-s} \Gamma(s)\left(\zeta(2 s)+O\left(y^{-\sigma}|s|^{-\varepsilon}\right)\right)} \\
= & \frac{\alpha(1-s)}{\alpha(s)} y^{1-2 s} \pi^{1 / 2} \frac{\zeta(2 s-1) \frac{\Gamma\left(s-\frac{1}{2}\right)}{\Gamma(s)}+\sum_{k=1}^{n} \frac{a_{k}}{2 \pi^{1 / 2}} \frac{\beta(s)}{\alpha(s)} L_{k}(s)}{\zeta(2 s)\left(1+O\left(y^{-2 \sigma}|s|^{-\varepsilon / 2}\right)\right)} \\
= & \frac{\alpha(1-s)}{\alpha(s)} y^{1-2 s} \pi^{1 / 2} \frac{\zeta(2 s-1) O\left(|s|^{-\frac{1}{2}}\right)+O\left(|s|^{-\varepsilon}\right)}{\zeta(2 s)\left(1+O\left(y^{-\sigma}|s|^{-\varepsilon / 2}\right)\right)} .
\end{aligned}
$$

Donc, pour $\sigma \geq 1 / 2,|s|$ assez grand

$$
F(s)=(-1)^{\operatorname{deg} \alpha}\left(1+O\left(|s|^{-1}\right)\right) y^{1-2 s} \pi^{1 / 2} \frac{\zeta(2 s-1) O\left(|s|^{-\frac{1}{2}}\right)+O\left(|s|^{-\varepsilon}\right)}{\zeta(2 s)\left(1+O\left(y^{-\sigma}|s|^{-\varepsilon / 2}\right)\right)} .
$$

a) Soit $\sigma_{0}>1 / 2$ suffisament grand, tel que $w(s) \neq 0$ et $F(s) \neq \pm 1$ sur $\sigma=\sigma_{0}$, et $\zeta(s) \asymp 1$ pour $\sigma \geq \sigma_{0}$. Lorsque $|\tau| \rightarrow+\infty, s=\sigma_{0}+i \tau$, on obtient de (33), $F(s)=O_{\sigma_{0}}\left(|\tau|^{-\varepsilon}\right)$, d'où la condition ( $i$ ") du théorème 24. Pour $1 / 2 \leq \sigma \leq \sigma_{0}$, la puissance $y^{-2 \sigma}$ est bornée; ceci avec $\zeta(2 s-1)=O\left(|\tau|^{A}\right)$ et $\zeta(2 s)^{-1}=O(\log |\tau|)$ nous donne $F(s)=O_{\sigma_{0}}\left(|\tau|^{A} \log |\tau|\right)$ pour $1 / 2 \leq \sigma \leq \sigma_{0}$, donc la condition (ii”) du théorème 24. Cela montre la proposition.

b) On suppose maintenant que $y \geq 1$. L'équation (32) se simplifie et on obtient

$$
w(s)=\alpha(s) y^{s} \zeta^{*}(2 s)\left(1+O\left(|s|^{-\varepsilon / 2}\right)\right)
$$

pour $\sigma \geq 1 / 2,|s|$ suffisament grand. Le nombre de zéros de $\alpha(s)$ étant fini, cela entraîne que $w(s)$ n'a qu'un nombre fini de zéros dans le demi-plan $\sigma \geq 1 / 2$. Pour $\sigma$ suffisament grand, (33) devient $F(s)=O\left(\sigma^{-\varepsilon}\right)$, d'où la condition (i) du théorème 4. Dans (a) on a montré une condition sufissante pour la condition (ii) du théorème 4, mais on peut montrer que $F(s)=O\left(|\tau|^{A} \log |\tau|\right)$ pour $\sigma \geq 1 / 2$ lorsque $y \geq 1$. L'application du théorème 4 nous donne le corollaire. 


\section{3 Étude en l'absence de symétrie réelle}

On veut généraliser les résultats obtenus jusqu'à présent pour des fonctions qui peuvent prendre des valeurs complexes sur la droite réelle. Pour traiter ce cas, étant donnée une fonction $h(s)$ méromorphe, définie sur un domaine $D \subset \mathbb{C}$, on définit

$$
\bar{h}(s)=\overline{h(\bar{s})}
$$

pour $s \in \bar{D}=\{\bar{z}, z \in D\}$. La fonction $\bar{h}(s)$ est aussi méromorphe. Si $h(s)$ est réelle sur la droite réelle, alors $\bar{h}(s)=h(s)$ pour $s \in D \cap \bar{D}$ par le principe de réflexion, pourvu que $D \cap \mathbb{R} \neq \emptyset$.

On veut maintenant étudier les fonctions

$$
f(s)=f^{ \pm}(s)=h(s) \pm \bar{h}(2 a-s),
$$

où $a \in \mathbb{R}$ est fixé. Ces fonctions réprésentent les parties réelle $\left(f^{+}(s)\right)$ et imaginaire $\left(f^{-}(s)\right)$ de la fonction $h(s)$, sur la droite critique $\sigma=a$. Au lieu des deux symétries de $f(s)$ on ne trouve qu'une seule, à savoir

$$
\bar{f}(2 a-s)= \pm f(s) .
$$

En analogie aux notations au $§ 1$, on note par

$$
\hat{N}(T)=\#\{s \in \mathbb{C}|f(s)=0,| \tau \mid<T\}
$$

le nombre de zéros de $f(s)$ avec $|\tau|<T$,

$$
\hat{N}_{0}(T)=\#\{s \in \mathbb{C}|f(s)=0, s=a+i \tau,| \tau \mid<T\}
$$

le nombre des mêmes zéros, sur la droite critique $\sigma=a$, et $\hat{N}_{0}^{\prime}(T)$ le nombre de ces derniers zéros, sans compter les multiplicités.

D'abord, on estime le nombre des zéros sur la droite critique, sans compter les multiplicités. La généralisation du lemme 2 est la suivante.

Lemme 31. Soit $a \in \mathbb{R}$ fixé, $h(s)$ une fonction méromorphe dans le plan complexe, réelle sur l'axe réel, sans zéros ni pôles sur la droite critique $\sigma=a$. On considère $\arg h(a+i \tau)$, une variation continue de l'argument de $h(s)$ sur la droite critique $\sigma=a$. Soit $f(s)=h(s) \pm \bar{h}(2 a-s)$. Le nombre de zéros de $f(s)$ avec $|\tau|<T$, sans compter les multiplicités, sur la droite $\sigma=$ a est minoré par

$$
\tilde{N}_{0}^{\prime}(T) \geq \frac{1}{\pi} \arg h(a+i T)-\frac{1}{\pi} \arg h(a-i T)-1 .
$$

Démonstration. On fait la preuve pour la fonction $f^{-}(s)$; en prenant $i h(s)$ au lieu de $h(s)$ on obtiendra $i f^{+}(s)$.

On peut supposer que $\arg h(a) \in]-\pi, 0]$. Si $f^{-}(a)=0$,

$$
\begin{aligned}
\hat{N}_{0}^{\prime}(T)= & \#\left\{\tau|| \tau \mid<T, \frac{1}{\pi} \arg h(a+i \tau) \equiv 0 \bmod 1\right\} \\
= & \#\left\{\tau \mid 0 \leq \tau<T, \frac{1}{\pi} \arg h(a+i \tau) \equiv 0 \bmod 1\right\} \\
& +\#\left\{\tau \mid 0 \leq \tau<T,-\frac{1}{\pi} \arg h(a-i \tau) \equiv 0 \bmod 1\right\}-1 .
\end{aligned}
$$

On obtient (34) par double application du lemme 1. De même si $f^{-}(a) \neq 0$.

Remarque. Dans le cas où $h(s)$ est réelle sur la droite réelle, on a $\tilde{N}_{0}^{\prime}(T)=2 N_{0}^{\prime}(T)+2 u_{ \pm}-1$ et $\arg h(a-i \tau)=-h(a+i \tau)$ (où $\arg h(a)=0$ ), d'où l'on retrouve le lemme 2 .

Finalement, on retrouve la généralisation du théorème 4, dont on ne fournit pas la preuve. 
Théorème 32. Soit $a \in \mathbb{R}, h(s)$ une fonction méromorphe sur $\mathbb{C}$, avec un nombre fini de pôles, un nombre fini de zéros dans le demi-plan $\sigma>a$, holomorphe et non nulle sur la droite critique $\sigma=a$. On définit

$$
f(s)=f^{ \pm}(s)=h(s) \pm \bar{h}(2 a-s)
$$

(en particulier $\bar{f}(2 a-s)= \pm f(s)$ ). On suppose que la fonction

$$
F(s)=\frac{\bar{h}(2 a-s)}{h(s)}
$$

satisfait

(i) pour chaque $\eta>0$, il existe $\sigma_{0}=\sigma_{0}(\eta)>$ a tel que $|F(s)|<\eta$ si $\sigma \geq \sigma_{0}, \tau \in \mathbb{R}$;

(ii) pour chaque $\varepsilon>0$ et $\sigma_{0}>a$, il existent deux suites $\left(T_{n}\right)_{n},\left(T_{n}^{*}\right)_{n}$ telles que $\lim _{n \rightarrow \infty} T_{n}=$ $\lim _{n \rightarrow \infty} T_{n}^{*}=+\infty$ et $|F(s)|<e^{\varepsilon|s|}$ pour $a \leq \sigma \leq \sigma_{0}$, et $\tau=T_{n}$ ou $\tau=-T_{n}^{*}, n \geq 1$.

Avec les notations du théorème 4.

$$
\hat{N}(T)-\hat{N}_{0}(T) \leq \hat{N}(T)-\hat{N}_{0}^{\prime}(T) \leq 1+2 P_{f, \sigma>a}+2 N_{h, \sigma>a}-2 P_{h, \sigma>a} .
$$

pour $T>0$. En particulier, presque tous les zéros de $f(s)$ se trouvent sur la droite $\sigma=a$ et ils sont simples. Le membre de gauche de (35) est de plus un nombre positif pair.

Remarque. Dans le cas où $h(s)$ est réelle sur la droite réelle, on a $\hat{N}(T)=2 N(T)+2 n_{f, \sigma>a}+$ $n_{f, a}$, ce qui avec la remarque faite ci-dessus, nous permet de retrouver le théorème 4 . Une conséquence immédiate de ce théorème est la répartition globale des zéros de $f(s)$ (comme dans le théorème 6).

Théorème 33. Sous les conditions du théorème 32, si $\arg h(a+i t)$ est une détermination continue de l'argument de $h(s)$ sur la droite $a+i \mathbb{R}$, alors

$$
\hat{N}_{0}^{\prime}(T)=\frac{1}{\pi} \arg h(a+i T)-\frac{1}{\pi} \arg h(a-i T)+O(1) .
$$

Les zéros des fonctions $f(s)=h(s) \pm \bar{h}(2 a-s)$ sont ultimement simples et entrelacés.

Corollaire 34. Sous les conditions du théorème [32, si h(s) est une fonction entière, alors

$$
\hat{N}(T)-\hat{N}_{0}(T) \leq \hat{N}(T)-\hat{N}_{0}^{\prime}(T) \leq 1+2 N_{h, \sigma>a} .
$$

On appliquera ces résultats à l'étude des translatées des fonctions $L$ complétées sur $\mathbb{Q}$ au lieu des fonctions $\zeta^{*}(s)$ ou $\xi(s)$ au $\S 7$. Malheureusement, l'estimation de $\hat{N}(T)-\hat{N}_{0}^{\prime}(T)$ obtenue n'est pas optimale. En fait, si $h(s)$ n'a pas de zéro dans le demi-plan $\sigma \geq a$, dans le dernier corollaire $N_{h, \sigma>a}=0$, d'où $\hat{N}(T)=\hat{N}_{0}(T)$, ce qui nous dit que tous les zéros de $f(s)$ sont alignés sur la droite $\sigma=a$, mais par contre on a seulement que $\hat{N}_{0}(T)-\hat{N}_{0}^{\prime}(T) \leq 1$, ce qui indique que tous les zéros de $f(s)$ sont simples, sauf peut-être un. Dans le cas réel (théorème 4), la symétrie additionnelle règle ce problème. Dans le cas général, il s'applique la remarque (c,1) faite au théorème 4, et la proposition 39 montre la simplicité de tous les zéros de $f(s)$.

Si l'on oublie pour l'instant la nécessité de montrer la simplicité des zéros de $f(s)$, on peut affaiblir les conditions sur $h(s)$, comme dans le $\$ 5$.

Corollaire 35. Soit $a \in \mathbb{R}, h(s)$ une fonction entière, avec un nombre fini de zéros dans le demiplan $\sigma \geq a$, sans zéros sur la droite $\sigma=a$. Avec les notations du thèorème 32, si la fonction $F(s)$ satisfait

(i') il existent $\sigma_{0} \geq a, C>0$ tels que $|F(s)| \leq C$ si $\sigma \geq \sigma_{0}, \tau \in \mathbb{R}$,

et la condition (ii) du théorème, alors pourvu que la fonction $f^{ \pm}(s)=h(s) \pm \bar{h}(2 a-s)$ soit non nulle,

$$
\hat{N}(T)-\hat{N}_{0}(T) \leq 2 N_{h, \sigma>a} .
$$


Démonstration. Par la remarque (a) au théorème 4, on peut éliminer les zéros (en nombre fini) sur la droite $\sigma=a$; la procédure ne change pas la quantité $\hat{N}(T)-\hat{N}_{0}(T)$. On introduit la famille de fonctions

$$
H(y ; s)=y^{s} h(s) \pm y^{2 a-s} \bar{h}(2 a-s)
$$

pour le paramètre $y>1$. Il est évident que $f(s)=\lim _{y \rightarrow 1^{+}} H(y ; s)$ uniformément dans les compactes de $\mathbb{C}$. La fonction

$$
F(y ; s)=y^{2 a-2 s} \frac{\bar{h}(2 a-s)}{h(s)}=y^{2 a-2 s} F(s)
$$

satisfait maintenant la condition (i) du théorème 32. En plus, les zéros de $y^{s} h(s)$ sont les mêmes que ceux de $h(s)$. Donc

$$
\hat{N}(y ; T)-\hat{N}_{0}(y ; T) \leq \hat{N}(y ; T)-\hat{N}_{0}^{\prime}(y ; T) \leq 1+2 N_{h, \sigma>a} .
$$

où les quantités $\hat{N}(y ; T), \hat{N}_{0}(y ; T)$ et $\hat{N}_{0}^{\prime}(y ; T)$ sont les analogues de $\hat{N}(T), \hat{N}_{0}(T)$ et $\hat{N}_{0}^{\prime}(T)$, respectivement, en fonction du paramètre $y$. Dans cette dernière inegalité double, le membre à gauche est un nombre entier pair, et celui à droite un entier impair, donc

$$
\hat{N}(y ; T)-\hat{N}_{0}(y ; T) \leq 2 N_{h, \sigma>a} .
$$

Pour $T$ fixé, le théorème de Hurwitz nous donne, pour $y>1$ assez proche de 1 ,

$$
\hat{N}(T)-\hat{N}_{0}(T) \leq \hat{N}(y ; T)-\hat{N}_{0}(y ; T) \leq 2 N_{h, \sigma>a} .
$$

Cela finit la preuve.

Le corollaire 35 peut être appliqué à un polynôme complexe quelconque; on peut comparer ce résultat avec ceux de de Bruijn, cfr. [7, Lemma 2, Theorem 9A].

On se demande s'il est possible d'améliorer l'estimation obtenue dans le théorème 4 pour avoir un résultat aussi précis que dans le cas réel. Pour réduire d'une unité l'estimation (35) dans un cas non trivial (non couvert par la proposition 39), le premier pas est de remarquer que l'estimation (34) reste valable en remplaçant le membre à droite par

$$
\tilde{N}_{0}^{\prime}(T) \geq\left\lceil\frac{1}{\pi} \arg h(a+i T)\right\rceil+\left\lceil-\frac{1}{\pi} \arg h(a-i T)\right\rceil-1 .
$$

Soit $\psi(\tau)=\frac{1}{\pi} \arg h(a+i T)$. Dans la démonstration du théorème 4, l'estimation de $R(T)-N_{0}^{\prime}(T)$ fait apparaitre $\psi(\tau)-\lceil\psi(\tau)\rceil$, ce que normalement on borne trivialement par 0. On peut garder cet écart; sa contribution à l'estimation de la borne (35) est la moyenne $\frac{1}{T} \int_{0}^{T}(\psi(\tau)-\lceil\psi(\tau)\rceil) d \tau$. On réénonce cela en termes de la fonction partie fractionnaire $\{x\}=x-\lfloor x\rfloor$. On sait que $\{x\}=$ $1+x-\lceil x\rceil$ pour $x \notin \mathbb{Z}$, donc $\{\psi(\tau)\}=1+\psi(\tau)-\lceil\psi(\tau)\rceil$ presque partout (les zéros de $h(s)$ étant isolés), et l'estimation triviale est $\{\psi(\tau)\} \leq 1$.

Question. Sous quelles conditions sur $h(s)$, la fonction $\psi(\tau)$ satisfait-elle

$$
0 \leq \limsup _{T \rightarrow+\infty} \frac{1}{T} \int_{0}^{T}\{\psi(\tau)\} d \tau<1 ?
$$

On fournit deux conditions suffisantes sur $\psi(\tau)$ :

(i) Soit $\psi(\tau)$ différentiable, $c_{1}, c_{2}>0$ tels que

$$
0<c_{1} \leq \psi^{\prime}(\tau) \leq c_{2}
$$

pour $\tau$ suffisament grand, alors

$$
\frac{c_{1}}{2 c_{2}} \leq \liminf _{T \rightarrow+\infty} \frac{1}{T} \int_{0}^{T}\{\psi(\tau)\} d \tau \leq \limsup _{T \rightarrow+\infty} \frac{1}{T} \int_{0}^{T}\{\psi(\tau)\} d \tau \leq 1-\frac{c_{1}}{2 c_{2}} .
$$


(ii) Soit $\psi(\tau)$ convexe et croissante pour $\tau$ suffisament grand, alors

$$
\limsup _{T \rightarrow+\infty} \frac{1}{T} \int_{0}^{T}\{\psi(\tau)\} d \tau \leq \frac{1}{2}
$$

La condition (i) peut être appliquée pour établir une extension de la proposition 8 : la condition est satisfaite par un polynôme complexe $p(s)$ quelconque, lorsque $y>1$.

La condition (ii) permet d'étendre les résultats du \$10, en particulier le théorème 18, au cas où $p(s)$ est un polynôme complexe. Les propriétés de croissance et convexité (dans un petit intervalle) de la fonction $\psi(\tau)=\frac{1}{\pi} \arg \left((i \tau) \zeta^{*}(1+2 i \tau) y^{i \tau}\right)$ (le cas $p(s)=1$ ) peuvent être vues dans [18, Lemma 2.3 ; en particulier $\psi^{\prime}(\tau) \sim \frac{1}{\pi} \log \tau$ et la condition (i) n'est pas satisfaite.

\subsection{Dominance faible et densité}

Les théorèmes 15 et 24 se généralisent de façon évidente; pour les généraliser on fournira au même temps une combinaison des deux.

On introduit quelques notations. Comme dans le $\$ 8$, pour une fonction $h(s)$ et $\sigma_{0}\left\langle\sigma_{1}, T>0\right.$, on note

$$
\tilde{N}_{h}\left(\sigma_{0}, \sigma_{1}, T\right)=\#\left\{s \in \mathbb{C}\left|h(s)=0, \sigma_{0} \leq \sigma \leq \sigma_{1},\right| \tau \mid<T\right\},
$$

et $\tilde{N}_{h}\left(\sigma_{0}, T\right)=\tilde{N}_{h}\left(\sigma_{0},+\infty, T\right)$.

De même, comme dans le §11, pour la fonction $f(s)=h(s) \pm h(2 a-s)$, on définit

$$
\hat{N}\left(T, \sigma_{0}\right)=\#\left\{s \in \mathbb{C}|f(s)=0,| \sigma-a\left|<\sigma_{0},\right| \tau \mid<T\right\} .
$$

En utilisant une extension simple du lemme 14, on démontre le résultat suivant, la version combinée des théorèmes 15 et 24 .

Théorème 36. Soit $\sigma_{0}>a, h(s)$ une fonction méromorphe, avec un nombre fini de pôles, holomorphe et non-nulle sur la droite $\sigma=\sigma_{0}$. On pose

$$
f(s)=f^{ \pm}(s)=h(s) \pm \bar{h}(2 a-s) .
$$

On suppose que la fonction

$$
F(s)=\frac{\bar{h}(2 a-s)}{h(s)}
$$

satisfait

(i') $F(s) \neq \pm 1$ sur la droite $\sigma=\sigma_{0}$, et il existe $\tau_{0}>0$ tel que $|F(s)|<1$ pour $\sigma=\sigma_{0},|\tau| \geq \tau_{0}$;

(ii') il existe une fonction croissante $\phi: \mathbb{R} \rightarrow \mathbb{R}$, une constante $K>0$ et des suites $\left(T_{n}\right)_{n},\left(T_{n}^{*}\right)_{n}$ telles que $\lim _{n \rightarrow \infty} T_{n}=\lim _{n \rightarrow \infty} T_{n}^{*}=+\infty$,

$$
T_{n} \leq T_{n+1} \leq \phi\left(T_{n}\right), \quad T_{n}^{*} \leq T_{n+1}^{*} \leq \phi\left(T_{n}^{*}\right), \quad \text { pour } n \geq 1,
$$

et $|F(s)|<e^{K|s|}$ pour $a \leq \sigma \leq \sigma_{0}$, et $\tau=T_{n}$ ou $\tau=-T_{n}^{*}, n \geq 1$.

Alors, pour $T>0$

$$
\hat{N}\left(T, \sigma_{0}\right)-\hat{N}_{0}^{\prime}(T) \leq 4 \hat{N}_{h}\left(a, \sigma_{0}, \phi(2 T)\right)+O(1) .
$$

\subsection{Translatées des fonctions $L$ de Dirichlet}

Soit $L(s, \chi)$ une série de Dirichlet associée à un caractère de Dirichlet primitif de conducteur $N>1$,

$$
L(s, \chi)=\sum_{n=1}^{\infty} \frac{\chi(n)}{n^{s}}
$$


pour $\sigma>1$. On associe à cette série la fonction completée $\xi(s, \chi)$, définie par

$$
\xi(s, \chi)=\left(\frac{N}{\pi}\right)^{\frac{s}{2}} \Gamma\left(\frac{s+\kappa}{2}\right) L(s, \chi),
$$

où

$$
\kappa=\frac{1}{2}(1-\chi(-1))= \begin{cases}0, & \text { si } \chi(-1)=1 \\ 1, & \text { si } \chi(-1)=-1\end{cases}
$$

La fonction $\xi(s, \chi)$ est une fonction entière de genre 1 qui satisfait à l'équation fonctionnelle [14, Theorem 4.15]

$$
\xi(s, \chi)=\epsilon(\chi) \xi(1-s, \bar{\chi}),
$$

où $\epsilon(\chi)=i^{-\kappa} \frac{\tau(\chi)}{\sqrt{N}}, \tau(\chi)$ est une somme de Gauss, de sorte que $|\epsilon(\chi)|=1$. Les zéros de $\xi(s, \chi)$ sont sur la bande $0<\sigma<1$. L'hypothèse de Riemann pour $L(s, \chi)$ est la conjecture que tous les zéros de $L(s, \chi)$ dans la bande $0<\sigma<1$ sont sur la droite $\sigma=1 / 2$.

On va réproduire les résultats du $\S 7$ et du $\S 9$ pour les fonctions $L(s, \chi)$. On a besoin de l'analogue du lemme 16 pour la fonction $L(s, \chi)$.

Lemme 37. Soit $L(s, \chi)$ la série de Dirichlet associée à un caractère primitif de conducteur $N>1$.

1. Il existe une constante $A>0$ telle que, pour chaque $\varepsilon>0,1 / 2<\sigma \leq 1, T \geq 3$

$$
\hat{N}_{L(\cdot, \chi)}(\sigma, T)=O\left(\left(T^{(2+\varepsilon)(1-\sigma)}+T^{c(\sigma)(1-\sigma)}\right) \log ^{A} T\right),
$$

où $c(\sigma)=\min \left(\frac{3}{2-\sigma}, \frac{3}{3 \sigma-1}\right)$.

2. Il existe une constante $A>0$ telle que pour chaque $n \geq 1$, il existe $n<T_{n}<n+1$ tel que

$$
|L(\chi, s)|>\tau^{-A}
$$

lorsque $\tau=T_{n},-1 \leq \sigma \leq 2$.

\section{Démonstration.}

1. C'est un cas particulier du théorème de densité dans [14, §10.4, p. 260].

2. On considère la formule dans [3, p. 102]

$$
\frac{L^{\prime}(\chi, s)}{L(\chi, s)}=\sum_{\rho,|\tau-\gamma| \leq 1} \frac{1}{s-\rho}+O(\log |\tau|)
$$

valable pour $-1 \leq \sigma \leq 2,|\tau| \geq 2$, la somme portant sur les zéros $\rho=\beta+i \gamma$ de $L(s, \chi)$ avec $|\tau-\gamma| \leq 1$. L'intégration de cette formule entre $s=\sigma+i \tau$ et $2+i \tau$ nous donne

$$
\log L(\chi, s)=\sum_{\rho,|\tau-\gamma| \leq 1} \log (s-\rho)+O(\log |\tau|)
$$

pour $-1 \leq \sigma \leq 2,|\tau| \geq 2$. À partir d'ici, la méthode de démonstration du théorème équivalent pour la fonction zêta de Riemann, dans [34, §9.7], nous donne le résultat.

On peut maintenant énoncer le résultat attendu. On remarque que le cas (1) avec un polynôme constant $p(s)$ à été établi par Lagarias [19, Lemma 5.1, Theorem 5.1], qui au même temps a determiné la distribution limite des espacements des zéros ces fonctions.

Théorème 38. Soit $\alpha>0$. Soit $\xi(s, \chi)$ une fonction $L$ complétée associée à un caractère de Dirichlet $\chi$ primitif de conducteur $N>1, p(s)$ un polynôme complexe sans zéro sur la droite $\sigma=1 / 2$. On considère la fonction

$$
f_{p, \chi, \alpha}^{ \pm}(s)=p(s) \xi(s+\alpha, \chi) \pm \bar{p}(1-s) \xi(s-\alpha, \chi) .
$$

Alors 
1. Pour $\alpha \geq 1 / 2$,

$$
\hat{N}(T)-\hat{N}_{0}^{\prime}(T) \leq 2 N_{p, \sigma>1 / 2}+1,
$$

où $N_{p, \sigma>1 / 2}$ est le nombre de racines de $p(s)$ dans le demi-plan $\sigma>1 / 2$. Donc tous les zéros de $f_{p, \chi, \alpha}^{ \pm}(s)$, sauf un nombre fini, sont sur la droite $\sigma=1 / 2$, ils sont simples et entrelacés. De même pour $0<\alpha<1 / 2$ sous l'hypothèse de Riemann pour $L(s, \chi)$.

Dans le cas particulier où $N_{p, \sigma>1 / 2}=0$, tous les zéros des fonctions $f_{p, \chi, \alpha}^{ \pm}(s)$ sont sur la droite $\sigma=1 / 2$, ils sont simples et entrelacés.

2. Pour $0<\alpha<1 / 2$,

$$
\hat{N}(T)-\hat{N}_{0}^{\prime}(T) \leq 4 \hat{N}_{L(\cdot, \chi)}\left(\frac{1}{2}+\alpha, 2 T+4\right)+O(1) .
$$

En particulier, il existe une constante $A>0$ telle que, pour tout $\varepsilon>0$,

$$
\hat{N}(T)-\hat{N}_{0}^{\prime}(T)=O\left(\left(T^{(2+\varepsilon)\left(\frac{1}{2}-\alpha\right)}+T^{k(\alpha)\left(\frac{1}{2}-\alpha\right)}\right) \log ^{A} T\right),
$$

pour $T>0$, où $k(\alpha)=\min \left(\frac{6}{3-2 \alpha}, \frac{6}{1+6 \alpha}\right)$.

Démonstration. Si dans l'équation fonctionnelle pour $L(s, \chi)$ on écrit $\epsilon(\chi)=e^{2 i \theta}$, alors

$$
e^{-i \theta} f_{\chi, \theta, \alpha}^{ \pm}(s)=e^{-i \theta} p(s) \xi(s+\alpha, \chi) \pm e^{i \theta} \bar{p}(1-s) \xi(1-s+\alpha, \bar{\chi}) .
$$

On vérifiera les conditions des théorèmes 32 et 36 . On pose

$$
F(s)=e^{2 i \theta} \frac{\bar{p}(1-s)}{p(s)} \cdot \frac{\xi(s-\alpha, \chi)}{\xi(s+\alpha, \chi)} .
$$

Par la formule de Stirling, pour $\sigma \geq 1 / 2,|s|$ suffisament grand

$$
F(s)=\omega\left(\frac{\pi}{N}\right)^{\alpha} s^{-\alpha}\left(1+O\left(|s|^{-1}\right)\right) \frac{L(s-\alpha, \chi)}{L(s+\alpha, \chi)},
$$

où $|\omega|=1$. Pour la série de Dirichlet $L(s, \chi)=1+O\left(2^{-\sigma}\right)$ pour $\sigma$ suffisament grand, puis

$$
|F(s)|=O\left(\sigma^{-\alpha}\right),
$$

donc la condition (i) du théorème 32 .

D'un autre côté, la fonction $L(s, \chi)$ est polynomialment bornée dans un demi-plan $\sigma \geq \sigma_{\alpha}=$ $1 / 2-\alpha$ [14, Lemma 5.2], c'est-à-dire $L(s, \chi)=O\left(|\tau|^{A_{0}}\right)$ avec $A_{0}=A_{0}\left(\sigma_{\alpha}\right)$. Le lemme 37 nous donne une constante $A_{1}>0$ et une suite $\left(T_{n}\right)_{n}$ telles que $n<T_{n}<n+1,|L(s, \chi)|^{-1}<|\tau|^{A_{1}}$ pour $-1 \leq \sigma \leq 2, \tau=T_{n}, n \geq 1$. En appliquant le même lemme à la fonction $L(s, \bar{\chi})$, on obtient $A_{2}>0$ et une suite $\left(T_{n}^{*}\right)_{n}$ tels que $n<T_{n}^{*}<n+1$, et $|L(s, \chi)|^{-1}=|L(\bar{s}, \bar{\chi})|^{-1}<|\tau|^{A_{1}}$ pour $-1 \leq \sigma \leq 2$, $\tau=-T_{n}^{*}, n \geq 1$. Le rassemblement de ces conditions nous donne

$$
|F(s)|=O\left(|\tau|^{B}\right),
$$

pour $\sigma \geq 1 / 2, \tau=T_{n}$ ou $\tau=-T_{n}^{*}, n \geq 1$, d'où la condition (iii) du théorème 32, ainsi que la condition (ii $)$ du théorème 36 pour $\sigma_{0}>2$ quelconque et $\phi(T)=T+2$, comme dans la proposition 17. Le premier théorème (avec la remarque (c.1) au théorème 4 et la proposition 39 dans le cas où $N_{p, \sigma>1 / 2}=0$ ) nous donne (1), le deuxième (2), et l'estimation dans (2) vient directement de la première partie du lemme 37 . 


\section{A Stabilité}

Par souci de complétude, on va mentionner des résultats concernant le problème de la stabilité d'un point de vue plus classique. Soit $h(s)$ une fonction entière et $a \in \mathbb{R}$; on dira que $h(s)$ est stable par rapport à la droite $\sigma=a$, si tous ses zéros sont dans le demi-plan $\sigma<a$ (et tout simplement stable si $a=0)$.

On réénonce un résultat originalement dû à de Branges [6, Lemma 5], puis retrouvé par Lagarias dans [19, Lemma 2.2].

Proposition 39. Soit $h(s)$ une fonction entière stable par rapport à la droite $\sigma=a$, et telle que la fonction

$$
F(s)=\frac{\bar{h}(2 a-s)}{h(s)}
$$

satisfait

$$
|F(s)|<1 \text { pour } \sigma>a \text {. }
$$

Alors

1. les fonctions $f(s)=f^{ \pm}(s)=h(s) \pm \bar{h}(2 a-s)$ ont tous leurs zéros sur la droite $\sigma=a$;

2. toute fonction de phase $\varphi(\tau)=\arg h(a+i \tau)$, est une fonction strictement croissante. Donc, les zéros des fonctions $f^{ \pm}(s)$ sont simples et entrelacés.

Démonstration. En fait, $\sigma>a,|f(s)| \geq|h(s)|(1-|F(s)|)>0$, puis par l'équation fonctionnelle, pour $\sigma<a$, on a $2 a-\sigma>a$ et $|f(s)|=|\bar{f}(2 a-s)|=|f(2 a-\bar{s})|>0$. Cela montre (1). On renvoit le lecteur à la démonstration [19, Lemma 2.2] pour la partie (2). On avait dejà remarqué l'entrelacement des zéros dans ce cas, au §2.

Un argument simple montre qu'un polynôme stable non constant (cfr. 5) satisfait les conditions de la proposition 39. Soit $p(s)=a_{0} \prod_{\rho}(s-\rho)$ la factorisation de $p(s)$, et $F(s)=\bar{p}(-s) / p(s)$. On a

$$
|F(s)|=\left|\frac{\bar{p}(-s)}{p(s)}\right|=\prod_{\rho}\left|\frac{-s-\bar{\rho}}{s-\rho}\right|=\prod_{\rho}\left|\frac{s+\bar{\rho}}{s-\rho}\right| .
$$

Soit $\rho=\beta+i \gamma$ un zéro de $p(s)$; on a $\beta<0$. Pour $s=\sigma+i \tau$, l'inégalité $|s+\bar{\rho}|<|s-\rho|$, ou

$$
(\sigma+\beta)^{2}+(\tau-\gamma)^{2}<(\sigma-\beta)^{2}+(\tau-\gamma)^{2}
$$

est équivalente à $4 \sigma \beta<0$. Puisque $\beta<0$, on doit avoir $\sigma>0$. Cela montre que l'inégalité $|F(s)|<1$ est satisfaite pour chaque terme du produit.

Par la remarque (c,1) faite au théorème 4, valable pour une fonction sans la symétrie réelle, si $h(s)$ est une fonction stable par rapport à la droite $\sigma=a$ qui satisfait les conditions du théorème 32, alors elle satisfait les conditions de la proposition 39, et on obtient les mêmes conclusions dans les deux théorèmes pour les fonctions $f^{ \pm}(s)$, voire mieux avec la proposition 39 . Si la fonction $h(s)$ n'est pas stable, la remarque (c,2) au théorème nous donne une condition avec laquelle on ne peut rien dire sur les zéros des fonctions $f^{ \pm}(s)$; il est là où le théorème 32 donne une réponse.

On veut étendre la condition de la proposition 39 à des fonctions entières. La procédure menée pour les polynômes est aussi valable pour les fonctions de genre 0 [21, Part I, §4.2], qui se factorisent de la même façon que les polynômes. Mais les fonctions qu'on rencontre dans les applications, telles que la fonction $\xi(s)$ de Riemann ou les fonctions $L$, sont de genre 1, et possèdent une factorisation moins simple.

Le résultat suivant, est établi par Suzuki et Lagarias dans [20, Theorem 4, §2] pour des fonctions réelles sur la droite réelle. Il peut être comparé au résultat classique de Pólya [24, Hilfssatz II]. 
Théorème 40. Soit $h(s)$ une fonction entière de genre 0 ou 1, qui satisfait une équation fonctionnelle de la forme

$$
\bar{h}(2 a-s)=e^{i \theta} h(s)
$$

pour $0 \leq \theta<2 \pi$, et telle que ses zéros sont inclus dans une bande de la forme

$$
|\sigma-a|<b,
$$

où $b>0$, et $\alpha \geq b$. Alors

$$
\left|\frac{h(s-\alpha)}{h(s+\alpha)}\right|<1 \text { pour } \sigma>a .
$$

Démonstration. Sans perte de généralité, on peut traiter le cas $a=0$. On supposera initialement que $h(0) \neq 0$. On considère la factorisation de Weierstrass de $h(s)$ [21, Part I, §4.2] (le choix $a=0$ simplifie le calcul; en général on prendrait la factorisation de $h(a+s)$ au lieu de celle de $h(s))$

$$
h(s)=e^{A+B s} \prod_{\rho}\left(1-\frac{s}{\rho}\right) e^{s / \rho},
$$

où $\sum_{\rho} \frac{1}{|\rho|^{2}}<+\infty,|\rho|<b$ pour tout $\rho$. L'équation fonctionnelle $\bar{h}(-s)=e^{i \theta} h(s)$ entraîne que si $h(\rho)=0$, alors $h(-\bar{\rho})=e^{-i \theta} \overline{h(\rho)}=0$. Puis, on peut régrouper les zéros $\rho=\beta+i \gamma$ par des blocs $B(\rho)=\{\rho,-\bar{\rho}\}$ pour $\beta \neq 0, B(\rho)=\{\rho\}$ pour $\beta=0$. Cela nous donne

$$
h(s)=e^{A+B s} \prod_{\substack{B(\rho) \\ \beta \neq 0}}\left(1-\frac{s}{\rho}\right)\left(1+\frac{s}{\bar{\rho}}\right) e^{s(1 / \rho-1 / \bar{\rho})} \prod_{\substack{\rho \\ \beta=0}}\left(1-\frac{s}{\rho}\right) e^{s(1 / \rho)}
$$

et $1 / \rho-1 / \bar{\rho}=-2 i \gamma /|\rho|^{2}$. Donc, on peut redistribuer les constantes et écrire

$$
h(s)=e^{A+B s} \prod_{\rho}\left(1-\frac{s}{\rho}\right) e^{c(\rho) s},
$$

où $c(\rho)=-i \gamma /|\rho|^{2}$ (ce qui est important ici est le fait que $\Re(c(\rho))=0$ ), et le produit (ainsi que tous les produits dorénavant) est conditionnellement convergent,

$$
\prod_{\rho}\left(1-\frac{s}{\rho}\right) e^{c(\rho) s}=\lim _{T \rightarrow \infty} \prod_{|\rho|<T}\left(1-\frac{s}{\rho}\right) e^{c(\rho) s}
$$

La derivée logarithmique de $h(s)$ (de (37)) évaluée en $s=0$ est $h^{\prime}(0) / h(0)=B$, et par l'équation fonctionnelle

$$
B=\frac{h^{\prime}(0)}{h(0)}=-\frac{h^{\prime}(-0)}{h(-0)}=-B
$$

d'où $\Re(B)=0$.

Puis, pour $\alpha \geq b, s=\sigma+i \tau$

$$
\left|\frac{h(s-\alpha)}{h(s+\alpha)}\right|=\prod_{\rho}\left|\frac{1-\frac{s-\alpha}{\rho}}{1-\frac{s+\alpha}{\rho}}\right|=\prod_{\rho}\left|\frac{\rho-s+\alpha}{\rho-s-\alpha}\right|
$$

et encore par la symétrie des zéros

$$
\left|\frac{h(s-\alpha)}{h(s+\alpha)}\right|=\prod_{\rho}\left|\frac{\rho-s+\alpha}{-\bar{\rho}-s-\alpha}\right| .
$$


On montrera que chacun des termes du produit satisfait l'inégalité attendue. L'inégalité

$$
|\rho-s-\alpha|<|-\bar{\rho}-s+\alpha|
$$

et équivalente à

$$
(\beta-\sigma-\alpha)^{2}+(\gamma-\tau)^{2}<(-\beta-\sigma+\alpha)^{2}+(\gamma-\tau)^{2}
$$

ou $(\beta-\alpha) \sigma<0$. Comme $\beta-\alpha<b-\alpha \leq 0$, alors on a (38) si et seulement si $\sigma>0$. Ceci nous donne la conclusion du théorème dans ce cas.

Si $s=0$ est un zéro d'ordre $m$ de $h(s)$, on peut écrire $h(s)=s^{m} h_{1}(s)$, où $h_{1}(0) \neq 0, \overline{h_{1}}(-s)=$ $e^{i \theta_{1}} h(s)\left(\theta_{1}=\theta\right.$ si $m$ est pair, $\theta_{1}=\theta \pm \pi$ si $m$ est impaire) et on peut obtenir les inégalités attendues pour $h_{1}(s)$. De plus

$$
\left|\frac{s-\alpha}{s+\alpha}\right|<1 \text { pour } \sigma>0
$$

ce qui avec l'inégalité pour $h_{1}(s)$ nous donne le résultat.

En vue de l'équation fonctionnelle, le théorème 40 établit que la fonction $h(s+\alpha)$ vérifie les conditions de la proposition 39. Maintenant on décrit rapidement certaines applications de combiner ces résultats. On garde la notation du théorème 40 pour $a=\frac{1}{2}, \alpha$ et $b$.

1. On pose $F(s)=\xi\left(2 s-\frac{1}{2}\right)$, ici $a=\frac{1}{2}, b=\alpha=\frac{1}{4}$. La fonction $\xi(2 s)=F\left(s+\frac{1}{4}\right)$ satisfait les conditions de la proposition 39, et on obtient le corollaire 20, l'hypothèse de Riemann pour la fonction zeta de Weng de rang 2. De même on peut obtenir la partie (ii) du théorème 18. Il est pour cet objectif que le théorème 40, appliqué à une fonction réelle sur la droite réelle, a été introduit par Lagarias et Suzuki en [20].

2. La fonction $\xi(s+\alpha)$ satisfait les conditions de la proposition 39 inconditionellement pour $\alpha \geq \frac{1}{2}\left(a=\frac{1}{2}, b=\frac{1}{2}\right.$ ), et sous l'hypothèse de Riemann pour $\alpha>0$ (dans ce cas $b$ est quelconque). On obtient les résultats sur les fonctions $\xi(s+\alpha) \pm \xi(s-\alpha)$ dont on a discuté à la fin du $\S 7$.

3. Les fonctions $\xi(s+\alpha, \chi)$, associées aux charactères primitifs de conducteur $N>1$, satisfont les conditions de la proposition 39 inconditionellement pour $\alpha \geq \frac{1}{2}$, et sous l'hypothèse de Riemann pour $L(s, \chi)$ pour $\alpha>0$. Ces fonctions ont été aussi considerées par Lagarias dans [19]. La combinaison de ces fonctions avec des polynômes dont les racines sont dans le demi-plan $\sigma<1 / 2$ nous donne le cas particulier dans la première partie du théorème 38 .

4. La fonction de Bessel $K_{s}(A)(a=0)$. Dans l'article [24], Pólya localise d'abord les zéros de cette fonction à l'aide de la formule 29, dans la bande $|\sigma|<1=b$, pour montrer après qu'ils sont simples et alignés avec les relations (22).

\section{Remerciements}

Je remercie Michel Balazard, directeur de ma thèse de doctorat, dont ce travail fait partie, pour les conseils opportuns, et Arnaud Chadozeau, pour des discussions concernant le $\$ 13$.

\section{Références}

[1] R. Bellman et K. L. Cooke : Differential-difference equations. Academic Press, New York, 1963.

[2] Н. Г. Чеботарев et Н. Н. Мейман : Проблема Рауса-Гурвица для полиномов и целых

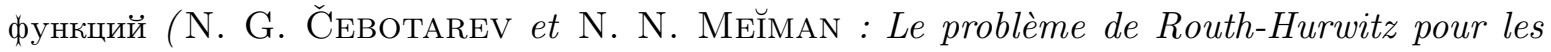
polynômes et les fonctions entières), vol. 26. Trudy Mat. Inst. Steklov., 1949. 
[3] H. Davenport : Multiplicative number theory, vol. 74 de Graduate Texts in Mathematics. Springer-Verlag, New York, third édn, 2000. Revised and with a preface by Hugh L. Montgomery.

[4] H. Davenport et H. Heilbronn : On the zeros of certain Dirichlet series. Proc. London Math. Soc., 11(3):181-185, 1936.

[5] H. Davenport et H. Heilbronn : On the zeros of certain Dirichlet series, second paper. Proc. London Math. Soc., 11(4):307-312, 1936.

[6] L. De Branges : Some Hilbert spaces of entire functions. Proc. Amer. Math. Soc., 10(5):840$846,1959$.

[7] N. G. de Bruijn : The roots of trigonometric integrals. Duke Math. J., 17:197-226, 1950.

[8] H. M. Edwards : Riemann's zeta function. Dover Publications Inc., Mineola, NY, 2001. Reprint of the 1974 original [Academic Press, New York].

[9] D. R. Grayson : Reduction theory using semistability. Comment. Math. Helv., 59(4):600-634, 1984.

[10] T. HAYASHI : Computation of Weng's rank 2 zeta function over an algebraic number field. $J$. Number Theory, 125:473-527, 2007.

[11] D. A. Hejhal : On a result of G. Pólya concerning the Riemann $\xi$-function. J. Analyse Math., 55:59-95, 1990.

[12] O. Holtz : Hermite-Biehler, Routh-Hurwitz, and total positivity. Linear Algebra Appl., 372:105-110, 2003.

[13] A. Ivić : The Riemann zeta-function. A Wiley-Interscience Publication. John Wiley \& Sons Inc., New York, 1985. The theory of the Riemann zeta-function with applications.

[14] H. Iwaniec et E. Kowalski : Analytic number theory, vol. 53 de American Mathematical Society Colloquium Publications. American Mathematical Society, Providence, RI, 2004.

[15] H. KI : On a theorem of Levinson. J. Number Theory, 107(2):287-297, 2004.

[16] H. KI : All but finitely many non-trivial zeros of the approximations of the Epstein zeta function are simple and on the critical line. Proc. London Math. Soc. (3), 90(2):321-344, 2005.

[17] H. KI : On the nontrivial zeros of modified Epstein zeta functions. C. R. Math. Acad. Sci. Paris, 342(2):79-81, 2006.

[18] H. KI : Zeros of the constant term in the Chowla-Selberg Formula. Acta Arith., 124(3):197204, 2006.

[19] J. C. Lagarias : Zero spacing distributions for differenced L-functions. Acta Arith., 120(2): 159-184, 2005.

[20] J. C. Lagarias et M. Suzuki : The Riemann hypothesis for certain integrals of Eisenstein series. J. Number Theory, 118(1):98-122, 2006.

[21] B. Y. Levin : Lectures on entire functions, vol. 150 de Translations of Mathematical Monographs. American Mathematical Society, Providence, RI, 1996. In collaboration with and with a preface by Yu. Lyubarskii, M. Sodin and V. Tkachenko, Translated from the Russian manuscript by Tkachenko.

[22] N. Levinson : On theorems of Berlowitz and Berndt. J. Number Theory, 3:502-504, 1971.

[23] W. MÜLLER : A spectral interpretation of the zeros of the constant term of certain Eisenstein series, 2007. À apparaître au Journal de Crelle.

[24] G. PÓLYA : Bemerkung Über die Integraldarstellung der Riemannschen $\zeta$-Funktion. Acta Math., 48(3-4):305-317, 1926. 
[25] G. Pólya et G. Szegő : Problems and theorems in analysis. I. Classics in Mathematics. Springer-Verlag, Berlin, 1998. Series, integral calculus, theory of functions, Translated from the German by Dorothee Aeppli, Reprint of the 1978 English translation.

[26] М. М. Постников : Устойчивые многочлены (M. M. Postnikov : Polynômes Stables). Nauka, Moscou, 1981.

[27] H. S. A. Potter et E. C. Titchmarsh : The zeros of Epstein's zeta functions. Proc. London Math. Soc. (2), 39(1):372-384, 1935.

[28] W. RUdin : Real and complex analysis, 3rd ed. McGraw-Hill, Inc., New York, NY, USA, 1987.

[29] A. Selberg et S. Chowla : On Epstein's zeta-function. J. Reine Angew. Math., 227:86-110, 1967.

[30] M. SuzukI : The Riemann hypothesis for the Weng zeta function of rank 3 for the rationals. In Conference on L functions (ed. by M. Kaneko and L. Weng), p. 175-199. World Scientific, 2007.

[31] P. R. TAYLOR: On the Riemann zeta function. Quart. J. Math., Oxford Ser., 16:1-21, 1945.

[32] The PARI Group, Bordeaux. PARI/GP, version 2.3.2, 2007. Available from http://pari.math.u-bordeaux.fr/.

[33] E. C. Titchmarsh : The theory of Functions. The Clarendon Press Oxford University Press, New York, second édn, 1939.

[34] E. C. Titchmarsh : The theory of the Riemann zeta-function. The Clarendon Press Oxford University Press, New York, second édn, 1986. Edited and with a preface by D. R. HeathBrown.

[35] S. M. Voronin : On the zeros of zeta-functions of quadratic forms. Tr. Mat. Inst. Steklova, 142:135-147, 1976. English translation.

[36] L. Weng : A rank two zeta and its zeros. J. Ramanujan Math. Soc., 21(3):205-266, 2006.

[37] D. ZAGIER : The Rankin-Selberg method for automorphic functions which are not of rapid decay. J. Fac. Sci. Univ. Tokyo Sect. IA Math., 28(3):415-437 (1982), 1981. 